\title{
The Investigation of Motor Primitives During Human Reaching Movements and the Quantification of Post-Stroke Motor Impairment
}

\author{
Erienne Virginia Olesh
}

Follow this and additional works at: https://researchrepository.wvu.edu/etd

\section{Recommended Citation}

Olesh, Erienne Virginia, "The Investigation of Motor Primitives During Human Reaching Movements and the Quantification of Post-Stroke Motor Impairment" (2017). Graduate Theses, Dissertations, and Problem Reports. 6346.

https://researchrepository.wvu.edu/etd/6346

This Dissertation is protected by copyright and/or related rights. It has been brought to you by the The Research Repository @ WVU with permission from the rights-holder(s). You are free to use this Dissertation in any way that is permitted by the copyright and related rights legislation that applies to your use. For other uses you must obtain permission from the rights-holder(s) directly, unless additional rights are indicated by a Creative Commons license in the record and/ or on the work itself. This Dissertation has been accepted for inclusion in WVU Graduate Theses, Dissertations, and Problem Reports collection by an authorized administrator of The Research Repository @ WVU.

For more information, please contact researchrepository@mail.wvu.edu. 


\title{
The investigation of motor primitives during human reaching movements and the quantification of post-stroke motor impairment.
}

\author{
Erienne Virginia Olesh
}

\author{
Dissertation submitted \\ To the School of Medicine, \\ Blanchette Rockefeller Neurosciences Institute \\ At West Virginia University
}

In partial fulfillment of the requirements for the degree of

Doctor of Philosophy in

Neuroscience

Department of Human Performance

Morgantown, West Virginia

2017

Keywords: Motor primitives, motor control, stroke, rehabilitation

Copyright 2017 Erienne Olesh 


\section{Abstract \\ The investigation of motor primitives during human reaching movements and the quantification of post-stroke motor impairment.}

\section{Erienne Virginia Olesh}

Movement is a complex task, requiring precise and coordinated muscle contractions. The forces and torques produced during multi-segmental movement of the upper limbs in humans, must be controlled, in order for movement to be achieved successfully. Although a critical aspect of everyday life, there remain questions regarding the specific controller used by the central nervous system to govern movement. Furthermore, how this system is affected by neurological injuries such as stroke also remains in question. It was the goal of this thesis to examine the neurological control of movement in healthy individuals and apply these findings to the further investigation of chronically motor impaired stroke patients. Additionally, this work aimed at providing clinicians with a more reliable, easy to use, and inexpensive approach to quantify post-stroke motor impairment. 
1.1. REVIEW OF LITERATURE

1.2. BACKGROUND OF MOTOR CONTROL RESEARCH

1.3. MOTOR CONTROL DESCRIBED THROUGH MOTOR PRIMITIVES

1.4. MOTOR PRIMITIVES AFTER STROKE

1.5. QUANTIFICATION OF POST-STROKE MOTOR IMPAIRMENT $\quad 7$

CHAPTER 2: AUTOMATED ASSESSMENT OF UPPER EXTREMITY MOVEMENT IMPAIREMENT DUE TO STROKE

2.1. ABSTRACT

2.2. INTRODUCTION

2.3. MATERIALS AND METHODS

2.4. RESULTS

2.5. DISCUSSION 16

2.6. FIGURES $\quad 18$

2.7. TABLES

2.8. REFERENCES

CHAPTER 3: AUTOMATED ASSESSMENT OF THE WOLF MOTOR FUNCTION TEST 29

3.1. INTRODUCTION

3.2. METHODS

3.3. RESULTS AND CONCLUSIONS

$\begin{array}{ll}\text { 3.4. FIGURES } & 33\end{array}$

CHAPTER 4: GRAVITATIONAL SIGNALS UNDERLIE TONIC MUSCLE ACTIVITY DURING

GOAL-DIRECTED REACHING

4.1. ABSTRACT $\quad 36$

4.2. INTRODUCTION

4.3. MATERIALS AND METHODS

4.4. RESULTS

4.5. DISCUSSION

4.6. REFERENCES $\quad 48$

4.7. FIGURE LEGENDS

4.8. TABLES $\quad 54$

4.9. FIGURES $\quad 56$

CHAPTER 5: LINKING POST-STROKE MOVEMENT IMPAIRMENT TO MECHANISTIC CHANGES IN THE NEURAL CONTROL OF MOVEMENT

$\begin{array}{ll}\text { 5.1. INTRODUCTION } & 64\end{array}$

5.2. METHODS $\quad 66$

$\begin{array}{lr}\text { 5.3. RESULTS } & 69\end{array}$ 
5.4. DISCUSSION

5.5. FUTURE DIRECTIONS

CHAPTER 6: SUMMARY AND RECOMMEDNATIONS FOR FUTURE RESEARCH 76

6.1. INTRODUCTION 36

CHAPTER 7: REFERENCES 80 


\section{Chapter 1}

Introduction and Literature Review 


\section{Introduction}

We often take for granted the ease in which we are able to move through our environment. The complexity of producing well-controlled movement results from the careful control of our intricate, multi-joint musculoskeletal system. Included in this framework are 230 joints and 630 muscles, all of which must be coordinated in harmony during movement (Hollerbach and Flash, 1982). How our nervous system accounts for the various internal and external factors associated with movement is a complex question, and decades have been spent trying to provide answers. The anatomical location of motor control has also been a focus for researchers, who aim to better understand this complex system. The intertwining contributions to motor control from the cortex, cerebellum, and spinal cord all play critical roles and when one or multiple of these areas are damaged, movement can be greatly affected. Motor control research has employed a broad range of methodology including human biomechanical experiments to single unit recordings in primates and computational modeling of neurons. Despite these mountainous efforts, the controller responsible for our movement remains shrouded in uncertainty. 


\section{Review of Literature}

To appreciate the complexity of motor control, one must understand two distinct, but related aspects of motion: kinematics and dynamics. Kinematics refers to an objects motion, primarily its velocity and trajectory (Winter, 1990; An and Chao, 1985). Motion-capture technology and accelerometers are commonly used technologies, which allows for the recording of kinematic parameters such as position, velocity, and acceleration. This data can provide beneficial information regarding an individual's range of motion for specific joints or the overall biomechanical ability of a limb. In recent years, this type of analysis has greatly improved functional ability measurements in patients recovering from neurological injuries (Tura et al., 2012; Patel et al., 2009; Mathie et al., 2004). Although valuable for quantitative measurement purposes, it is somewhat arduous to infer control mechanisms from kinematics (Gribble and Ostry, 1999). This is because, by definition, kinematics does not include the forces that produce motion. In order to truly grasp the complexity of motor control and understand how our nervous system produces meaningful movements, we must also examine the dynamic properties (forces) that are both produced by and impact the human musculoskeletal system.

Unlike kinematics, dynamics includes the forces applied to an object, which in turn produce motion (Schneider et al., 1989). In regards to human movement, the term limb dynamics, encompass forces generated by our muscles, tendons and ligaments, as well as the interaction of forces between joints (Sainburg et al., 1999; Gribble and Ostry, 1999). The interaction of forces between joints, referred to as interaction torques, are a result of forces produced at one joint that passively affect movement at other joints (Hollerbach and Flash, 1982; Gribble and Ostry, 1999). These forces greatly increase the complexity of motor control by adding variables that fluctuate during movement (Hollerbach and Flash, 1982).

\section{Background of motor control research}

Prior to 1982, it was not well understood how the dynamic properties of the limb impacted human movement. Early research into human motor control had relied on extrapolating robotic and mechanical engineering principles in an attempt to explain human movement (Bizzi 2001; Hollerbach and Flash, 1981; Raibert, 1977). These early theories of motor control inferred that human motion was accomplished through mechanisms similar to the programming of movement in robotic systems (Raibert, 1977). However, these theories were lacking in their ability to account for sudden changes in the environment and provided no solution for online corrections or error feedback. The limitation of these theories was likely a result of experiments that relied heavily on the use of single-joint systems (Schneider et al., 1989). Single-joint experiments simplified the study of motor control by limiting the number of degrees of freedom but in turn, ignored the important dynamic aspects of motion (Gribble and Ostry, 1999; Hollerbach and Flash, 1982). With the emergence of multi-joint experiments came the increased understanding of the dynamical properties of limbs, and how these dynamic 
variables impacted motion (Hollerbach and Flash, 1982; Morasso 1982; Abend et al., 1982; Soechting and Lacquaniti 1981).

It became additionally apparent that the type of movement being performed, whether ballistic, accuracy dependent, or load bearing, also contributed to the overall impact of limb dynamics on the motion. During a ballistic movement, our upper extremities act much like a whip, causing the most distal point in the chain to move faster then the most proximal point (Bizzi 2001; Hollerbach and Flash, 1982). In respect to human movement, the shoulder represents the most proximal point, and our fingers the most distal point. During movement, forces produced at the shoulder propagate distally along the limb. These forces must be controlled for in order to properly stabilize the limb (Sergio and Kalaska, 1997). This can be easily demonstrated in the pitching motion of a baseball player. The torques, which originate from the shoulder girdle, travel along the limb and are conversely translated to the ball (Hamill and Knutzen, 2009). In expert baseball pitchers these forces are used to the pitchers advantage, allowing for high speed but controlled motion of the baseball (Hirashima et al., 2007). Even during small movements that require fine motor skills, interaction torques must still be accounted for in order to accomplish movement with accuracy (Hollerbach and Flash, 1982).

Despite the range in forces and torques that can arise during multi-segmental movements such as reaching, the underlying characteristics of those movements share important common features. Early experiments investigating the interaction of forces provided some of the first evidence suggesting the importance of accounting for kinematic and dynamic variables during motion. From these experiments two consistent findings became apparent; the linear trajectory of the hand and the associated bell shaped velocity profile (Morasso 1982; Abend et al., 1982; Soechting and Lacquaniti 1981; Atkenson and Hollerbach, 1985). Although these results may not be surprising they were significant for two reasons: 1 ) straight lines represent the shortest distance between two points and therefore are energetically favorable and 2) the linear path creates the smallest propagation of forces from the arm to the object being reached for (Hollerbach and Flash, 1982). Hollerbach and Flash argued that the only way the observed linearity in movement could be maintained was if forces and torques were properly compensated for during movement. These findings strongly argued for a control system that could account and predict both the kinematic and dynamic variables of movement.

Over the subsequent decades, numerous theories have been proposed that aim to provide a coherent explanation of how our central nervous system predicts, plans, and executes movement. As any debated topic, each theory has been supported through a variety of experimental procedures. The difficulty in comparing these theories and their accompanying research lies in the variability of human movement. Some theories may be better supported in the context of rhythmic movement such as walking; where as other theories have built their case using complex tasks such as reaching or the interaction between limbs and objects. One theory of motor control, which has received a great deal of attention and research, is that of motor primitives. In the next section we will discuss this theory and its application to studying motor impaired individuals. 


\section{Motor control described through motor primitives}

As mentioned previously, our nervous system must account for not only the bones, joints, and muscles in our body, but the resulting forces of these parts acting on each other. Understanding how our nervous system accounts for this wide array of variables, often referred to as the "degrees of freedom problem" or the problem of "motor abundance" has been central to motor control research. The notion that the central nervous system may reduce the complexity of the musculoskeletal system through discrete modules dates back to work from the early 1900's (Sherrington, 1910). Refining upon these early ideas, Nikoli Bernstein developed the theory of muscle synergies, which suggested that pre-set patterns of muscle activity can be actively combined to produce a variety of movement (Bernstein, 1967; Tresch and Bizzi, 1999). By having discreet sets of muscle activation patterns, the central nervous system would have a greatly reduced number of parameters to control, allowing for easier planning and execution of motion (Bizzi and Cheung, 2013; Bizzi 1991).

In the time since the conception of the muscle synergy theory, a great deal of research has investigated how these synergies are organized, and where anatomically, they may reside. Some of the evidence supporting the theory of muscle synergies comes from experiments of spinal stimulation in rats, frogs and cats demonstrating reproducible patterns of muscle activation when stimulating the same spinal loci (Bizzi et al., 1991; Tresch and Bizzi, 1999; Lemay and Grill, 2004). As the stimulating electrodes are moved to different locations in the lumbar spinal cord, different patterns of muscle activation can be visualized (Bizzi et al., 1991, Tresch et al., 2002). These patterns of muscle activation were found to be not only repeatable, but also produced meaningful movements such as swimming patterns in the frog and gait in the cat. Together, these experiments gave credence to the existence of muscle synergies in the spinal cord, and that perhaps, some aspects of limb dynamic control may be incorporated into the synergies (Bizzi and Cheung, 2013; Tresch et al., 2002; Tresch et al., 2006).

The identification of muscle synergies relies on finding co-variant patterns of muscle activation. This is most commonly done through applied algorithm procedures such as principal component analysis (PCA), non-negative matrix factorization (NMF), and independent component analysis (ICA) (Tresch, 2006). These methods have demonstrated accuracy in finding muscles that share common patterns of activation in both biological and artificial data sets (Steele et al., 2013). These "low-dimensional" subsets of muscle groupings are then termed a synergy, as they constitute specific patterns of muscle activation. The synergy itself, as defined by the algorithm used to identify them, has two discrete aspects; the weighted coefficients, and the temporal profile. The weighted coefficients are specific to each muscle within a given synergy and represent the relative strength in muscle activation for a given movement. The temporal profiles, on the other hand, are common to all muscles in the synergy but are scaled for each muscle based on that muscles weighted coefficient. This creates a temporal profile that represents the increase or decrease of activation of the synergy, while allowing for variations in specific muscle activity to accommodate the needs of different tasks. 
Interestingly, it appears that as research into muscle synergies has progressed, there has become perhaps a less concise view of what exactly defines a synergy. For instance, there have been examples of both time and time varying synergies (d'Avella et al., 2008; d'Avella et al., 2013). For the time invariant synergies there is no temporal delay in the activation of different muscles from one synergy. For time varying synergies however, there exists both a spatial and temporal component; the spatial component, constituting the pattern of activation across the muscles of the synergy and the temporal component, which drives the timing of the activations.

Uncertainty also exists in how the central nervous system "deploys" each synergy and how descending commands are organized to activate multiple synergies. Work by Ting and colleagues have suggested that multiple synergies may be co-activated in specifically weighted schemes to achieve task specific goals. The identification of synergies during these specific tasks, such as maintaining posture during a balance task, suggests that the groupings of muscles that make up a synergy are intentional to compensate for the biomechanical features of the body (Ting, 2007; Safavynia and Ting, 2012; Perreault et al., 2008; Torres-Oviedo and Ting, 2007). Other research has described the flexible scaling of the spatial and temporal components of synergies, allowing for a wide variety of movements to be performed (d'Avella and Lacquaniti, 2013).

Given the discourse over the specific purpose and location of muscle synergies, it seems appropriate that they are now, perhaps, more commonly referred to as motor primitives. In this context, a motor primitive is defined as a building block of the central nervous system which can be temporally, or spatially combined with other building blocks to produce meaningful and well controlled motion (Giszter, 2015). Exchanging the term motor primitive for muscle synergy also removes the exclusivity of only including muscles into these "building blocks". Motor primitives, as defined here, can be comprised of kinematic or dynamic variables (Grinyagin et al., 2005; Forner-Cordero et al., 2005) or potentially represent whole movement parameters (Bockemuhl et al., 2010). This broadened definition allows for greater investigation into the structure and function of these components and how they relate to the activation of muscle activity and motion as a whole. Not only has the investigation of motor primitives helped improve our understanding of motor control in healthy individuals, it has also allowed for the advancement of our knowledge regarding post stroke motor impairment.

\section{Motor Primitives after Stroke}

The investigation of changes in movement capabilities after a stroke has stemmed far beyond the world of physical therapists and clinicians and is now a commonly studied topic by motor control experts. One method of studying motor control changes in stroke patients has been through the quantification and exploration of motor primitives in patient populations. Although much of this work has referred to these primitives as muscle synergies, it again seems more appropriate to use the term motor primitives in lieu of muscle synergies. To clinicians, the term synergy is often seen in a negative light, as it implies improper movement of the limb caused by a lack in independent joint control (Dipietro et al., 
2006; Krakauer, 2005; Neckel et al., 2006). This loss of coordinated limb movement and subsequent inability to perform every day living tasks is the primary focus of rehabilitation experts when working with chronic motor impaired individuals. Regardless of the terminology used, the investigation of motor primitives in stroke patients has provided some beneficial insight into the underlying changes in muscle activities, which arise as a result of a stroke.

Clinically focused studies of stroke patients have often revolved around a patient's ability to perform sets of standardized movements. Although informative regarding a patients ability to perform activities of daily living (ADL's), these studies often overlook the mechanistic breakdown in the neural control of movement that leads to long-term movement dysfunction. Furthermore, it is often difficult to gage a patient's true muscular capability purely from kinematic parameters, such as joint angles or speed of movement, as neural deficits may be disguised by compensatory mechanisms of muscle activation (Safavynia et al., 2011). To navigate these issues, neuroscientists, often in collaboration with rehabilitation experts, have taken to studying the muscle activity patterns of stroke patients in an attempt to better understand the etiology of long-term motor impairment.

Studies investigating potential alterations to motor primitives in stroke patients have identified changes across mild, moderate and severely affected stroke patients (Cheung et al., 2009; Clark et al., 2010; Cheung et al., 2012; Roh et al., 2013; Roh et al., 2015). These observed changes have ranged from altered recruitment of motor primitives in mildly affected patients (Cheung et al., 2009) to the merging and fractionation of motor primitives in more severely affected patients (Cheung et al., 2012). Other researchers have likewise found that fewer motor primitives are required to produce movement on the more impaired side of the body when compared to the less impaired limb (Clark et al., 2010). Although dynamic reaching and locomotor tasks are more commonly used to study changes to primitive structure in stroke patients, alterations of primitive structure have also been captured during isometric tasks (Roh et al., 2013; Roh et al., 2015). By using changes in motor primitives as a descriptor of post stroke movement impairment, new insight into the underlying alterations of muscle activity has been provided, offering critical information about this vulnerable patient population.

\section{Quantification of post stroke Movement Impairment}

A great deal of research regarding the care and well being of stroke patients has focused on rehabilitation schemes and methods by which to quantify the effectiveness of those schemes in treating post stroke motor impairment. Typically, quantification of post stroke motor impairment has relied on measuring a patient's ability to perform an activity of daily living (ADL). These movements, such as brushing ones teeth, combing ones hair, or drinking from a cup, are often measured in terms of accuracy or time to complete. Tests that incorporate these movements such as the Fugl Meyer, the Action Research Arm Test, or the Wolf Motor Function Test, aim to provide a score of impairment for stroke patients that could be used by clinicians and physical therapists to guide rehabilitation efforts or predict the longterm outcome of movement ability. 
Unfortunately, these tests are often unused or disregarded as important clinical practices. Several shortcomings of these tests limit the practicality of use in a clinical setting. First, these tests often require specialized equipment and trained professionals to administer and score the test successfully. Second, these tests often require significant amounts of time to complete. Physical therapy sessions for chronic motor impaired patients are often limited by insurance standards to half or one hour sessions, therefor prohibiting the implementation of a test that could easily encompass the entire appointment time. Third and perhaps most importantly, these tests provide little information regarding the underlying causes of movement impairment. Motor impaired stroke patients often learn mechanisms of compensation, creating altered muscle strategies that allow them to perform a task in a manner that is similar to their pre-stroke capabilities. This could cause a stroke patient to receive a score indicating less impairment then is actually present.

One method to combat these issues is to provide rehabilitation specialists with true quantitative measures of motor impairment. This task has been recently taken up by motor control and biomechanical researchers. As mentioned previously, motor primitives have become a commonly implemented method to study and describe post stroke motor impairment. Other research however has focused on providing descriptive analysis of post stroke motor impairment in terms of kinematic and dynamic variables of movement (Levin, 1996; Beer et al., 2000; Dewald et al., 1995). Additionally, the novel application of biomechanical recording equipment such as accelerometers (Thies et al., 2009; Knorr et al., 2005) and motion capture equipment (Subramanian et al., 2010) has also been used in an attempt to study and quantify post stroke motor impairment. Yet, a convenient overlap between a description of the underlying motor impairment and an easy to use and understand score is still lacking.

The ability for rehabilitation specialists and clinicians to accurately quantify post stroke motor impairment is critical to the improvement of post stroke care. Without these defining measures, it is difficult to gage a patient's progress or response to specific treatments. These methods must be easy to use and clinically relevant while providing an encompassing view of each patient's motor ability. The ability to provide these measures, however, also relies on our ability to better understand the intricacies involved in movement and how the muscles of the body adapt to changing dynamic loads present through a range of kinematic tasks. This knowledge can then be applied to the study of stroke patients, which will hopefully lead to a better grasp on post stroke alterations to motor control that lead to chronic motor impairment. It was therefor the goal of this thesis work to not only investigate further into the structure and function of motor primitives and the underlying cause of post stroke motor impairment but also to provide a more quantitative approach for describing the motor function of stroke patients. 


\title{
Chapter 2
}

\author{
Automated Assessment of Upper Extremity Movement Impairment \\ Due To Stroke \\ Published: \\ August $6^{\text {th }}, 2014$ \\ PLOS ONE
}

doi: 10.1371/journal.pone.0104487

Title: Automated Assessment of Upper Extremity Movement Impairment Due To Stroke

Erienne V. Olesh ${ }^{1}$, Sergiy Yakovenko ${ }^{2}$, and Valeriya Gritsenko ${ }^{1}$

Affiliations:

${ }^{1}$ Division of Physical Therapy, School of Medicine, West Virginia University, Morgantown, WV.

2 Division of Exercise Physiology, School of Medicine, West Virginia University, Morgantown, WV.

Correspondence address:

Valeriya Gritsenko

PO Box 9226,

Morgantown, WV 26506

USA

Corresponding author:

Valeriya Gritsenko

email:vgritsenko@hsc.wvu.edu

fax: 304-293-7105

phone: 304-293-7719

Key words: motor, arm, outcome measure, motion capture, kinematics

Number of words: 6,173; number of tables: 2; number of figures: 5.

\section{Authors and Contributors}

EO contributed to the design of the study, subject recruitment, data collection, data analysis, and writing of the manuscript. SY contributed to the data collection and analysis, and to writing of the manuscript. VG contributed to the design of the study, analysis of data, and writing of the manuscript. 


\begin{abstract}
Current diagnosis and treatment of movement impairment post-stroke is based on the subjective assessment of select movements by a trained clinical specialist. However, modern low-cost motion capture technology allows for the development of automated quantitative assessment of motor impairment. Such outcome measures are crucial for advancing post-stroke treatment methods. We sought to develop an automated method of measuring the quality of movement in clinically-relevant terms from low-cost motion capture. Unconstrained movements of upper extremity were performed by people with chronic hemiparesis and recorded by standard and low-cost motion capture systems. Quantitative scores derived from motion capture were compared to qualitative clinical scores produced by trained human raters. A strong linear relationship was found between qualitative scores and quantitative scores derived from both standard and low-cost motion capture. Performance of the automated scoring algorithm was matched by averaged qualitative scores of three human raters. We conclude that low-cost motion capture combined with an automated scoring algorithm is a feasible method to assess objectively upper-arm impairment post stroke. The application of this technology may not only reduce the cost of assessment of post-stroke movement impairment, but also promote the acceptance of objective impairment measures into routine medical practice.
\end{abstract}




\section{Introduction}

Fifty percent of stroke survivors suffer from an impairment of motor function that requires prolonged rehabilitation [1,2]. Because the impairment of upper limb function is a predictor of long-term participation in activities of daily life [3] and quality of life post stroke [4], reduction of arm impairment is an important aspect of rehabilitation [5-7]. Rehabilitation programs for upper extremity are designed and delivered by physical or occupational therapists, based on their assessment of movement impairment. The success of this approach depends on the amount of experience and skillfulness of the therapist, and on the duration of treatment. However, there is no standard procedure for the assessment and treatment of the impairment in arm movement. This leads to the variability in the effectiveness of therapy and to the inability to compare interventions across practitioners and clinics. Furthermore, current consensus is that physical therapy continues to be effective months and years after a neurological damage, such as stroke [8-10]. However, with the current one-on-one hospital session approach, prolonged treatment is extremely expensive and usually does not last beyond the first month following a stroke. These limitations of current medical care create a strong motivation to deliver therapy at home [11]. Multiple home-based therapy systems are currently being developed world-wide [12-21].

To enable cross-evaluation of home-based treatments and help them move out of research realm into clinical practice, it is important to develop standard quantitative outcome measures that draw on the accumulated clinical experience of impairment assessment. The current state-of-the-art in clinical assessment of movement impairment is based on the subjective scoring of select movements by a trained clinical specialist. Several standard tests exist to assess the impairment of arm function, such as Fugl-Meyer Assessment (FMA)[22] and Action Research Arm Test (ARAT)[23] to name a few. These tests have established reliability, validity, and responsiveness values [24-29]. We propose to use validated clinical tests of movement impairment to develop an automated quantitative assessment of impairment. This will allow to not only standardize clinical impairment assessment, but also include it into home-based therapies and promote their cross-validation.

Recent technological improvements have resulted in low cost 3D motion capture systems such as Kinect Sensor (Microsoft). Such technology holds the potential of significantly advancing impairment assessment by providing objective kinematic data with which to guide the development of novel therapies (for review see [30]). Recent studies have shown that Kinect Sensor can be used to quantify clinically-relevant parameters of gait $[31,32]$ and posture $[33,34]$. Kinect-based virtual stepping therapy has been shown to be effective for post-stroke rehabilitation of gait [35]. Several recent pilot studies have also demonstrated that Kinect-based motion capture helps motivate neurological patients to participate in physical therapy [36], and that such therapy is well received by both patients and therapists $[37,38]$. However, quantitative assessment of arm impairment continues to be a challenge. To meet this challenge, we have developed the algorithm of automated clinical scoring for quantifying arm impairment. In this study we have tested this algorithm in its ability to quantify post-stroke upper extremity 
impairment from low-cost motion capture, and we compared its performance to that of trained human raters.

\section{Materials and Methods}

West Virginia University Institutional Review Board approved the protocol entitled A New Quantitative Biomechanical Method for Motor Assessment of Disability number 1311129283 . Prior to experiment, participants signed informed consent approved by the Institutional Review Board.

Participants

Study participants were adults with chronic hemiparesis with the following characteristics: 4 female, 5 male, $58 \pm 21$ years old, $5 \pm 6$ years post-stroke (standard deviation, s.d., is stated after \pm here and in the rest of the manuscript). They were medically stable and could comprehend simple instructions. Infarct locations were identified from MRI scans by the participant's care providers (Table 1). One subject was excluded from data analysis, because her self-report of stroke was not confirmed by her hospital chart.

\section{Procedures}

The participants performed 10 different arm movements (Fig. 1A) that are part of FMA[22] and ARAT[23]. The participants repeated each movement between 5 and 28 times after a demonstration by the experimenter. The movements were captured simultaneously by a standard motion capture system Impulse (Phase Space), the low-cost motion capture device Kinect Sensor (Microsoft), and recorded with a high-definition video camera (Samsung) for scoring by human raters. Movement selection was based on current capabilities of Kinect Sensor to track position of large arm segments, but not individual fingers.

Data were processed in Matlab (MathWorks, Inc.). The coordinates of multiple tracked skeletal landmarks were captured at $480 \mathrm{~Hz}$ by the standard system and at $30 \mathrm{~Hz}$ by the low-cost system (Fig. 1). These data were filtered using a second order Butterworth low-pass filter (cut-off at $6 \mathrm{~Hz}$ ). Next, we calculated four joint angles (shoulder flexion/extension, shoulder abduction/adduction, elbow flexion/extension, and wrist flexion/extension; termed kinematics) from motion capture data recorded by both systems during a single repetition of each of the 10 movements performed by the non-paretic and paretic limbs. Joint angles reflect independent degrees of freedom of the arm and, thus, encompass complex information about movement limitations of people post-stroke.

The temporal alignment of the corresponding movements for paretic and non-paretic arms was accomplished in three steps. Firstly, movement start and end was manually identified in a subset of data. Secondly, kinematic data aligned on these onsets were averaged per joint angle to create a mean trace, termed wavelet, for each movement kind. Lastly, the multiple movements per trial were identified using peaks in the correlation coefficient profile for different delays between joint angles and the wavelet. The time of peaks were further used to align movement repetitions within and across trials. Manual creation of the wavelet can be omitted in a fully automated version of this analysis, if a single movement is recorded per trial. 
Estimating minimal number of movement repetitions for low-cost assessment

We have used kinematics recorded by both systems to estimate the minimal number of movement repetitions required for sufficiently precise motion capture with the low-cost system. To accomplish this estimation we bootstrapped the data in several steps to estimate errors of averaging one, two, three, etc repetitions of the same movement. The errors were absolute differences between the maximal amplitude of angular motion in a single trial and the maximal amplitude of average angular motion across all corresponding trails. The following steps were carried out to bootstrap these errors: 1) To estimated the error from 1 repetition of the same movement, single-trial errors were drawn repeatedly and randomly with replacement from the dataset for each movement type and each participant. The average squared differences between the mean error and each of the single-trial errors was the estimate of error of low-cost motion capture during a single movement. 2) To estimate the error from 2 repetitions of the same movement, two single-trial error values were drawn repeatedly and randomly with replacement from the dataset for each movement type and each participant. The average squared differences between the overall mean error and the mean of two single-trial errors was the estimate of error of low-cost motion capture after two repetitions of a movement. 3) - 20) This bootstrapping was repeated with increasing number of trials (samples drawn from the population), until the maximal number of repetitions was reached for a particular movement and participant.

Lastly, we determined the first bootstrapped error value that fell below the 95\% confidence interval of the mean error for each movement and participant. The corresponding number of trials used to calculate this value of error indicated the minimal number of repetitions of the same movement needed for accurate motion capture by the low-cost system.

\section{Principle Component Analysis (PCA) for automated scoring of impairment}

Joint angles of the non-paretic arm of each subject were averaged across repetitions of the same movement, and principal components were derived from the averaged temporal profiles across the four joint angles using eigenvalue decomposition of the covariance matrix. Then, individual temporal profiles of the joint angles of paretic arm recorded during each repetition of each movement were reconstructed with the basis of the principal components derived from the averaged profiles of non-paretic arm. The number of principal components chosen for the reconstruction were sufficient to explain $\geqq 95 \%$ of variance in the kinematics. The reconstructed joint angle profiles were compared to the original paretic profiles using coefficient of determination $\left(\mathrm{R}^{2}\right)$, which indicated how closely non-paretic principal components represent the movement of paretic arm. Thus, this measure constitutes a quantitative score of impairment (WVU (C2012). The same decomposition was done on non-paretic data from individual trials using the principal components derived from the averaged non-paretic data. This measure showed the inherent variability of scoring using this method. The resulting $\mathrm{R}^{2}$ values for both of these analyses are plotted in Figure 2. 
The principal components were extracted from the demeaned joint angle profiles. The process of demeaning the data served to improve the quality of impairment assessment by removing inaccurate biases in the low-cost motion capture. Furthermore, the reduction of data dimensionality using principal component analysis also reduced the sensitivity of impairment assessment to noise in the low-cost motion capture data.

\section{Clinical scoring of impairment}

Thirty graduate students in the last year of their Degree of Physical Therapy generated standard qualitative scores by rating 5 repetitions of each movement from video recordings of study participants. Movements were rated on the FuglMeyer scale, 0 indicating no movement at all, 1 indicating slow and/or abnormal movement, and 2 indicating normal movement [22]. Students were instructed to follow this scale to the best of their ability. Intraclass correlation coefficient for the relationship between the mean group scores and each rater's scores was used to establish inter-rater reliability [39].

The strength of the relationship between the quantitative scores derived from standard and low-cost motion capture and between the quantitative and qualitative scores was determined using linear regression. The power of the Pearson correlation coefficient $(\beta)$ was determined from a statistical table [40]. Regression was also used to define the linear decoding model. The decoding performance of this linear model was evaluated by fitting regressions into data for all but one subject and then using this regression to predict the qualitative score of the subject that was left out. This was repeated for all 8 subjects.

\section{The number of raters that match performance of automated scoring}

To estimate how many human rates it would take to match automated scoring performance, we bootstrapped the qualitative scores in several steps similarly to the procedure described above. The qualitative scores of 30 human raters and quantitative scores from low-cost motion capture were used for this analysis. The mean qualitative score averaged across all raters represents the most accurate clinical measure of a participant's impairment. The average squared differences between the mean qualitative scores and the qualitative scores of each rater was the estimate of error of individual human raters. The rest of the qualitative scores were bootstrapped using the following approach. To compare the error of 2 human raters to the automated performance, qualitative scores produced by 2 human raters were drawn repeatedly and randomly with replacement from the dataset of qualitative scores for each movement type and each participant. The drawn values were averaged, subtracted from the overall mean qualitative scores and squared. The resulting population of qualitative score errors represented the estimate of errors of 2 human raters. This bootstrapping was repeated with increasing number of raters (samples drawn from the population), until the maximal number of 30 raters was reached for a particular movement and participant.

Lastly, we determined the first bootstrapped qualitative score error value that fell below the model performance error for each movement and participant. 
The corresponding number of raters used to calculate the value of qualitative score error indicated the minimal number of human raters it would take to surpass performance of the automated scoring algorithm.

\section{Results}

Quality of movement assessment using low-cost system

The quality of unconstrained 3D movements performed by each subject with paretic and non-paretic arm was automatically scored from kinematics. There was a strong linear relationship between the quantitative scores derived from both motion capture systems $\left(\mathrm{p}<0.001 ; \mathrm{R}^{2}=0.64 ;\right.$ Fig. $\left.3 \mathrm{~A}\right)$, indicating that they are analogous.

The standard clinical tests usually evaluate performance of single repetitions of different movements. To test the feasibility of using low-cost motion capture for clinical testing, we have estimated how many repetitions of the same movements it would take to achieve accurate kinematic data from the low-cost system. The number of repetitions of the same movement needed to obtain a mean estimate that falls within the $95 \%$ confidence interval was $1.98 \pm 0.50$ trials for shoulder abduction/adduction; $1.97 \pm 0.44$ trials for shoulder flexion/extension; $1.88 \pm 0.34$ trials for elbow flexion/extension; $1.85 \pm 0.48$ trials for wrist flexion/extension. This makes it feasible to use low-cost motion capture for fast automated testing.

\section{Qualitative scores vs. quantitative scores}

To score subject movements in clinically-relevant terms, we analyzed the motion capture data by converting it into physiological joint angles and applying PCA. More than $95 \%$ of variance across joint angles during the average movement of the non-paretic arm was represented by two principal components in all but one movement. These principal components could be used to reconstruct individual movements performed by both non-paretic and paretic arms with explained variances equal to $88.24 \pm 2.60 \%$ and $78.90 \pm 5.98 \%$ respectively. The quantitative scores based on the explained variances of paretic movements were linearly related to the qualitative scores $(p=0.001 ; \beta=0.97)$ with $R^{2}=0.868$ (Fig. 3B). The decoding performance of this linear model was characterized by the mean error of predicted scores being $7.68 \pm 7.52 \%$ of the maximal score (Fig. 4A). Regression offsets ranged from -1.94 to -1.24 , slopes ranged from 3.58 to 4.46 , and $\mathrm{R}^{2}$ ranged from 0.78 to 0.93 when individual participants were taken out of the dataset (Fig. 4B). This shows that it is feasible to automatically score movement impairment using low-cost motion capture.

\section{Consistency of human raters compared to quantitative scores}

We have used the average scores of human raters as the gold standard against which to compare our automatic scoring algorithm. However, the accuracy of human raters varies due to the subjective nature of this approach. The proposed quantitative analysis offers an accurate and unsupervised alternative to the subjective and time-consuming measures. The tuned scoring model has a comparative reliability of combined scores from 30 human raters in our study (Fig. $4 \mathrm{C})$. The algorithm used in this study performs as well as $3.42 \pm 1.78$ human raters 
(s.d. is across movements; Fig. 4D). This further supports the feasibility of using motion capture for automated scoring of movement impairment.

\section{Variability of scoring across different test movements}

PCA has shown that different movements typically included in clinical tests have different inter-trial variability. This is illustrated by the changes in the explained variance of decomposition based on mean principal components between different recorded movements (Fig. 2, top plot). This variability translates into variability of the relationship between qualitative and quantitative scores for each movement (Fig. 5). This suggests that some of the movements included in clinical tests may provide less reliable information about movement impairment because of their high inter-trial variability. Nevertheless, all relationships between quantitative and qualitative scores had positive slopes. This further supports our conclusion that using low-cost motion capture for automated scoring of movement impairment is feasible.

\section{Accuracy of low-cost motion capture}

The standard motion capture system was used as the gold standard to assess the kinematic accuracy of the low-end system. To compare the two systems we calculated the root mean squared (RMS) errors between them with single trials aligned on movement onset as described above. RMS errors were averaged across the duration of each movement and across the two limbs for each of the four physiological angles. In addition to the RMS errors, we have also calculated the absolute difference between maximal joint excursions for each movement captured by each of the systems. The mean errors of joint angles recorded by the low-cost motion capture system were considerable (Table 2). These errors are primarily due to biases, i.e. consistent over- or under-estimation of joint angles by the Kinect sensor due to inaccurate identification of tracked points on the body. Despite such large errors, quantitative assessment with PCA was successful in reproducing clinical assessment as shown above. This is because PCA is less sensitive to biases and noise in the motion capture data compared to RMS or movement excursion measures for reasons described above in the Methods section.

\section{Discussion}

The study results have shown that using low-cost motion capture with an automated scoring algorithm is a feasible method to assess objectively upper-arm impairment post stroke. Several recent studies have demonstrated the usefulness of whole-body kinematics in the assessment of improvements in post-stroke locomotion [41], arm-trunk coordination [42], and reaching movements [43]. Furthermore, motion capture was used to assess upper extremity motor function after constraint-induced movement therapy and was reported to have higher interrater reliability than possible with traditional clinical measures [44,45]. However, some major limitations of using motion capture for clinical needs is the cost, complexity, and lack of portability of traditional full body motion capture systems, which require several cameras and markers placed on subject's body. With the development of low-cost markerless 3D motion capture systems, such as the Kinect 
Sensor used in this study, out-of-the-lab movement kinematics with sufficient accuracy is now available for general use. The potential cost savings for clinics using the new low-cost motion capture technology are substantial, e.g. Kinect Sensor costs about $\$ 200$, while lab-based motion capture systems cost tens of thousands of dollars. However, the complexity of kinematic data is still a barrier to the widespread acceptance of it in clinical practice. Results of the current study aim to overcome this barrier by demonstrating the effectiveness of an automated algorithm to clinically assess arm impairment from kinematics. This allows for the automation of impairment assessment, which enables the inclusion of quantitative outcome measures in routine medical practice. Clinical automated assessments are already a reality for quantitative measures of gait and balance impairment using GAITRite (CIR Systems Inc) and SMART Balance Master (NeuroCom) respectively. The current study is the first to show that clinical assessment of arm motor impairment can be automated. The application of this technology may not only reduce the cost of assessment of post-stroke movement impairment, but also promote the acceptance of objective impairment measures into routine medical practice.

Results of our study have shown that automated quantitative assessment of movement impairment was as reliable as clinical assessment by thirty senior DPT students. This is consistent with previous studies showing that using motion capture for clinical assessment results in increased inter-rater reliability $[44,45]$. While inter-rater reliability between highly experienced therapist is likely to be higher, we believe that it is valid to compare automated performance against raters with variable levels of experience. This is because including raters with variable abilities is a more accurate representation of variance in skill in clinical practice. Overall, our results shows that automated scoring of motor impairment can increase the accuracy of clinical assessment. Furthermore, using a consistent algorithm for the analysis of kinematic data can help standardize outcome measures across medical specialists and across facilities.

Traditionally, clinical tests consist of different movements that are performed once by the patient. A single repetition of each movement is done to reduce the time it takes to perform the test, and thus reduce the time spent by a medical specialist on motor assessment. We have shown that to obtain reliable kinematics from Kinect Sensor, each movement has to be repeated three times. While this increases the time it takes for the patient to perform the test, averaging across repetitions of the same movements contributes to the increased reliability of motor assessment. Furthermore, the medical specialist will not need to be present during the test administration, thus his/her time spent on the assessment will be reduced. Therefore, we believe that it is feasible to implement the automated motor assessment in a clinical setting.

A limitation of the current study is that we employed a very coarse, although robust, 3-point clinical scale for the assessment of movement quality. Such scale has the resolution of $1 / 3$ or $33 \%$ of maximal range of motion. Therefore our data show that while the low-cost motion capture system is less accurate than the laboratory standard, it is more accurate than the 3-point clinical scale (Table 2). Future studies are needed to test the effectiveness of the PCA-based quantitative assessment in 
presence of biases and noise in the low-cost motion capture for scales with higher resolution and for more complex movements involving the hand.

Assessment of motor impairment using the FMA is useful for understanding the limitations in motion of individual joints and basic synergy patterns. However, to evaluate the effectiveness of rehabilitation in enabling people to return to their normal lives different kinds of movements prove more useful. For example, clinical tests of functional abilities such as Wolf Motor Function Test [46], rely on movements that mimic goal-directed tasks of daily living, e.g. picking up or manipulating household objects. Therefore, the next logical step for the development of quantitative assessment based on low-cost motion capture is to evaluate its effectiveness to extract information about the individual's function from such goal-directed movement.

\section{Figures}

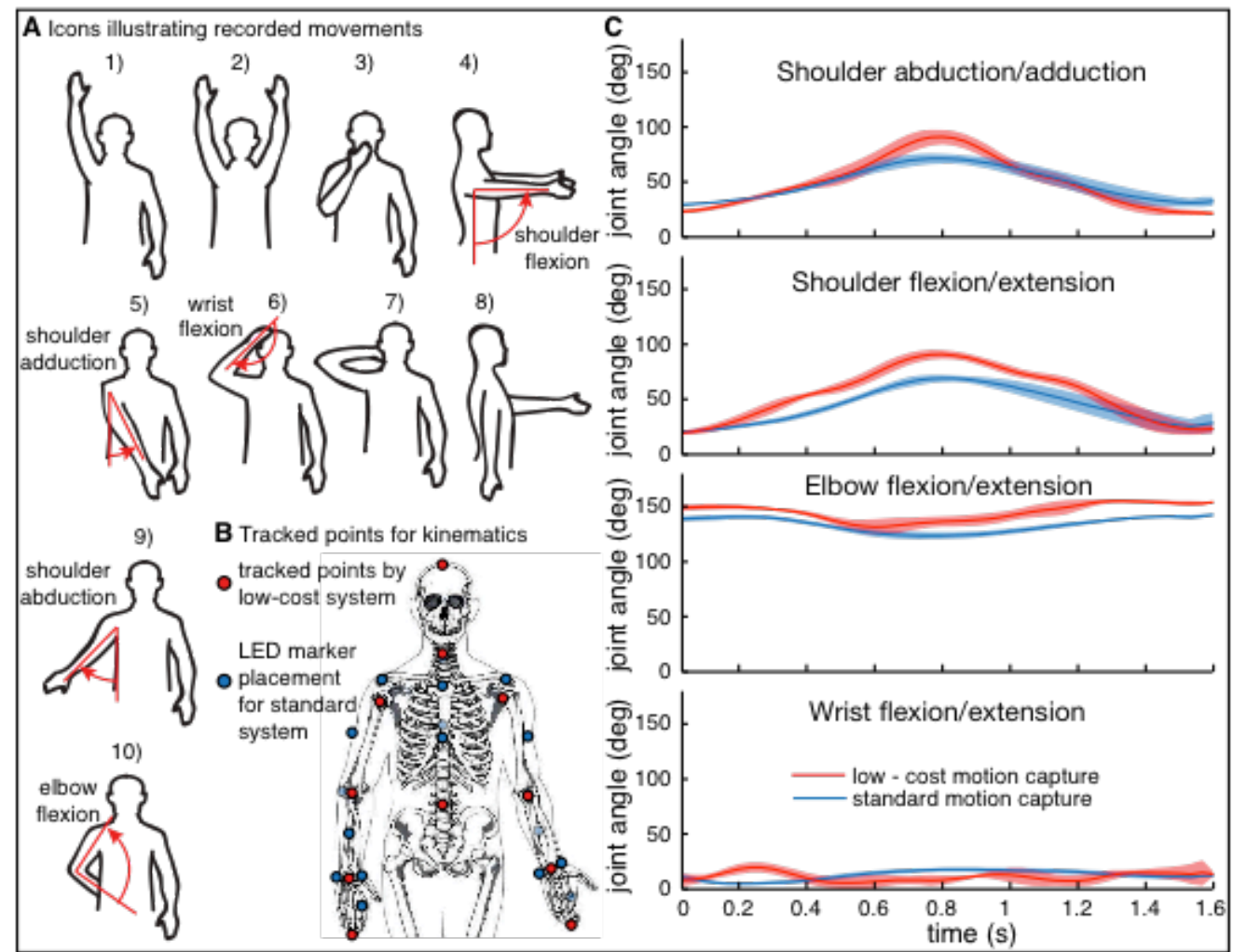

Figure 1. Study methodology. A, illustration of recorded movements. Red lines indicate the direction of motion for joint angles included in the kinematic analysis. B, tracked points used to calculate joint angles. C, Average joint angles of the left arm calculated from the data captured by the two motion capture systems during 10 repetitions of the movement 8 ) shown in A. 


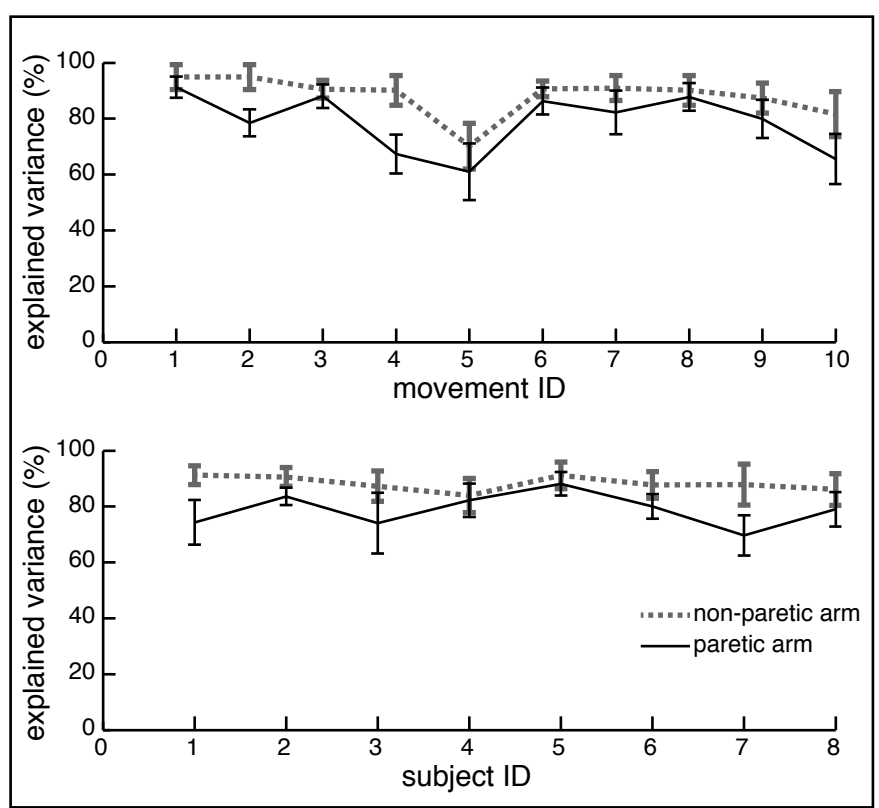

Figure 2. Results of principle component analysis. Cumulative explained variance and the number of principal components are shown for each movement type across participants (top plot) and for each participant across movement types (bottom plot). Grey dotted lines show results of decomposition of movement of the non-paretic arm, while black solid lines shows results of decomposition of movement of the paretic arm. The principal components were derived from mean data and used to reconstruct data from individual movements. 


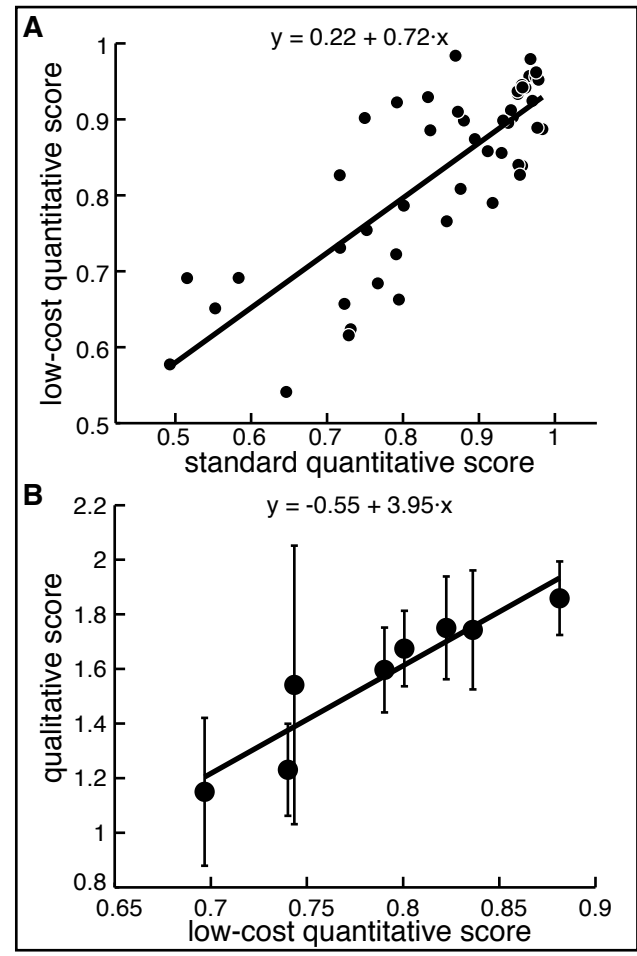

Figure 3. The comparison between quantitative scores from standard and low-cost motion capture and qualitative scores. A, Dots show mean scores for each movement and each subject; thick line shows a regression fit. B, Symbols show mean scores for each subject; error bars show s.d. across 10 movements; thick line shows a regression fit. 


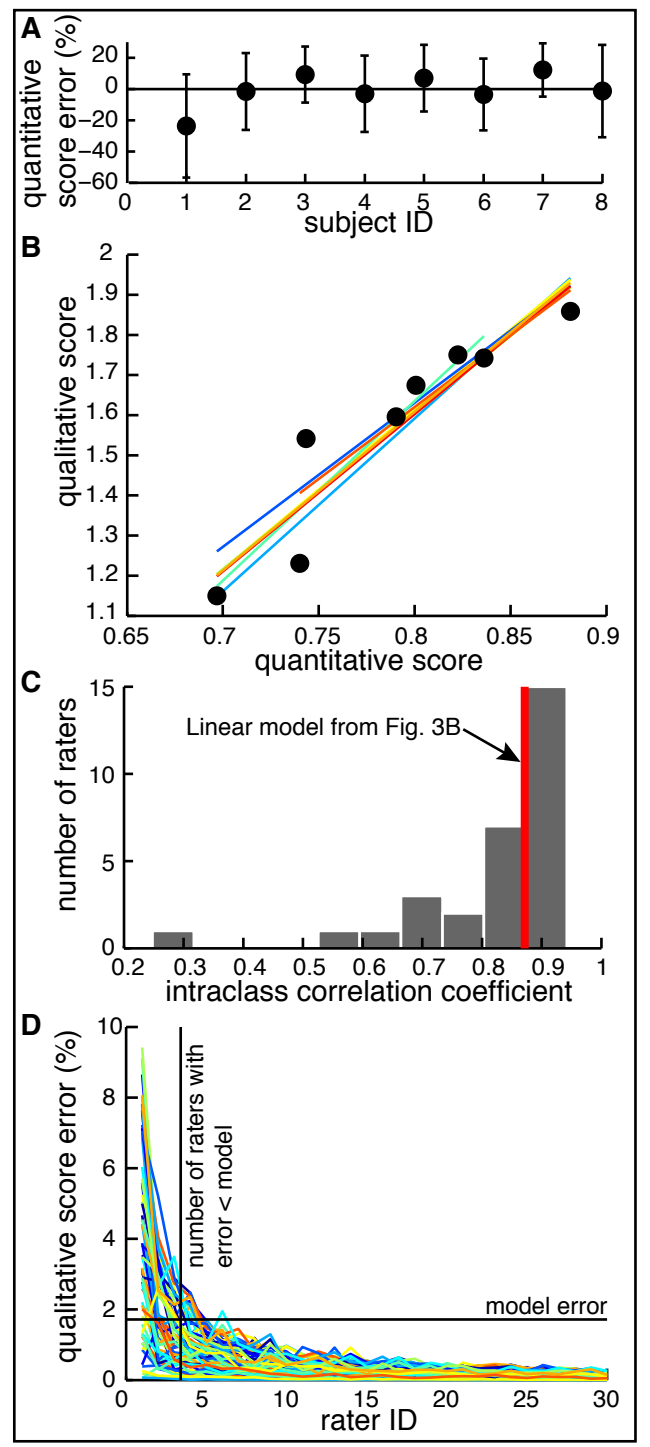

Figure 4. Decoding performance. A, Error in predicting each subject's qualitative score from regressions fitted to the rest of the participants. Mean errors are expressed as $\%$ of the correct score; error bars show s.d. across 10 movements. B, Symbols show the same data as in Fig. 3B; lines show regressions for datasets with one subject's data point removed. C, Histogram of intraclass correlation coefficients for relationships between individual human raters and the mean qualitative score. D, Colored lines show reducing errors as more raters score movements of the same participants per movement type, limb, and participant. 

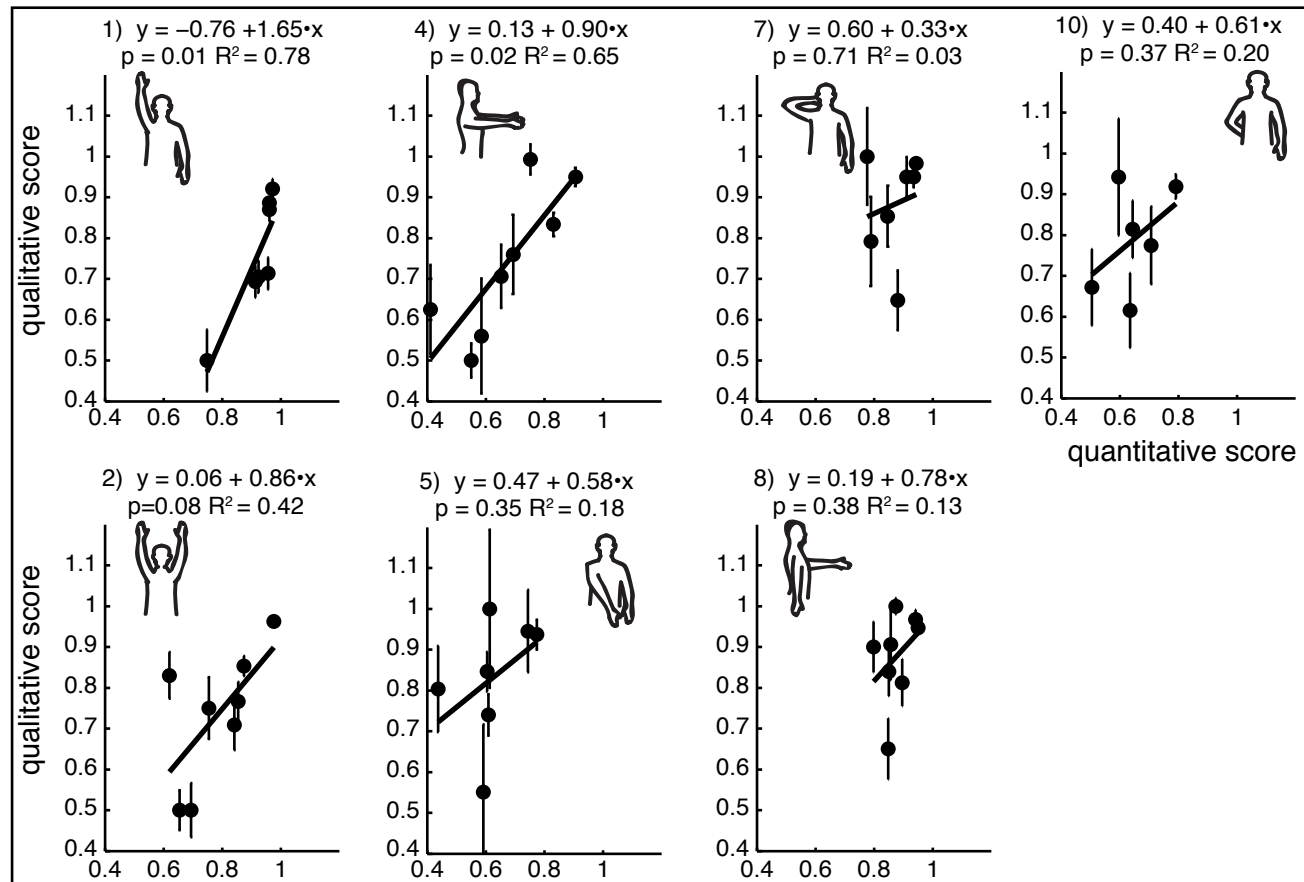

5) $y=0.47+0.58 \cdot x$

8) $y=0.19+0.78 \cdot x$
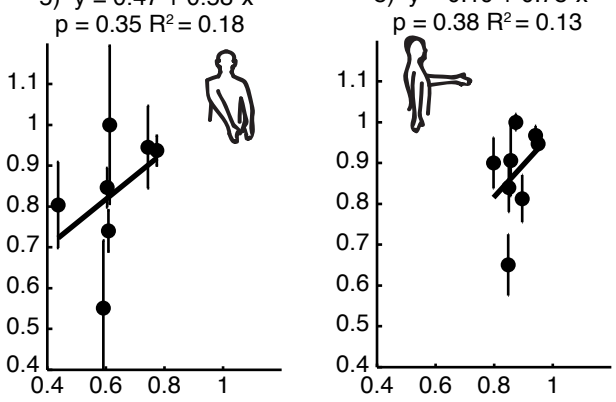
3) $\begin{aligned} y & =-1.47+2.62 \cdot x \\ p & =0.00 R^{2}=0.98\end{aligned}$

6) $y=-0.35+1.39 \cdot x$

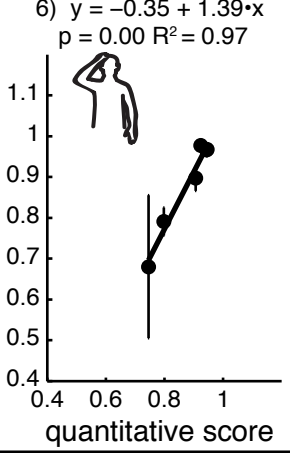

9) $\begin{gathered}y=-0.19+1.12 \cdot x \\ p=0.10 R^{2}=0.45\end{gathered}$

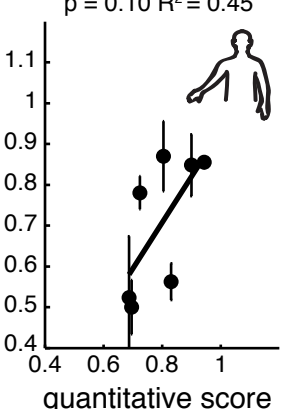

Figure 5. Relationships between qualitative and quantitative scores for each movement type. Dots show mean scores across participants, thick lines show linear regressions with their equations and fit statistics above each plot. 


\section{Tables}

Table 1: Summary of participant characteristics.

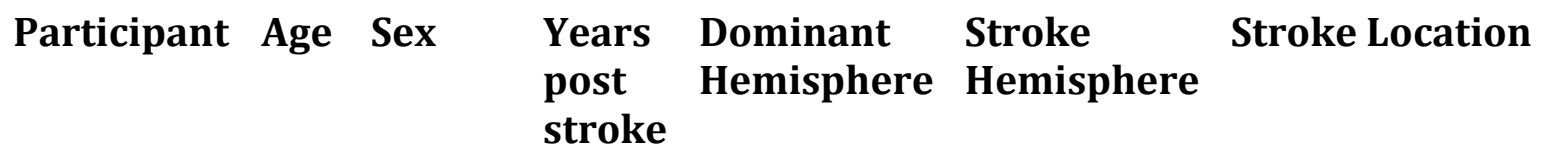

\begin{tabular}{|c|c|c|c|c|c|c|}
\hline 1 & 50 & Male & 5 & Right & Right & Caudal medulla \\
\hline 2 & 76 & Male & 2 & Left & Right & $\begin{array}{l}\text { Posterior globus } \\
\text { pallidus and internal } \\
\text { capsule }\end{array}$ \\
\hline 3 & 20 & Female & 20 & Right & Right & $\begin{array}{l}\text { Middle Cerebral } \\
\text { Artery distribution } \\
\text { involving portions of } \\
\text { frontal and temporal } \\
\text { lobes }\end{array}$ \\
\hline 4 & 80 & Female & 1 & Right & Left & $\begin{array}{l}\text { Posterior Limb of } \\
\text { Internal Capsule }\end{array}$ \\
\hline 5 & 62 & Male & 2 & Right & Right & $\begin{array}{l}\text { Frontal } \\
\text { intraparenchymal } \\
\text { hemorrhage }\end{array}$ \\
\hline 6 & 39 & Female & 1 & Right & Right & $\begin{array}{l}\text { Middle Cerebral } \\
\text { Artery distribution } \\
\text { involving portions of } \\
\text { frontal and parietal } \\
\text { lobes, putamen, and } \\
\text { globus pallidus }\end{array}$ \\
\hline 7 & 76 & Male & 4 & Right & Left & $\begin{array}{l}\text { Anterior temporal } \\
\text { lobe and posterior } \\
\text { left putamen }\end{array}$ \\
\hline 8 & 64 & Male & 4 & Right & Left & $\begin{array}{l}\text { Middle Cerebral } \\
\text { Artery distribution } \\
\text { involving portions of } \\
\text { frontal lobe }\end{array}$ \\
\hline
\end{tabular}


Table 2. Angular errors of low-cost motion capture relative to the standard system.

\begin{tabular}{|c|c|c|c|c|}
\hline & $\begin{array}{l}\text { shoulder } \\
\text { abduction/ } \\
\text { adduction angle }\end{array}$ & $\begin{array}{l}\text { shoulder } \\
\text { flexion/ } \\
\text { extension angle }\end{array}$ & $\begin{array}{l}\text { elbow } \\
\text { flexion/ } \\
\text { extension angle }\end{array}$ & $\begin{array}{l}\text { wrist } \\
\text { flexion/ } \\
\text { extension angle }\end{array}$ \\
\hline $\begin{array}{l}\text { Mean RMS } \\
\text { errors, } \\
\text { degrees }\end{array}$ & $22.03 \pm 9.55$ & $25.81 \pm 10.57$ & $22.88 \pm 8.15$ & $15.99 \pm 7.41$ \\
\hline $\begin{array}{l}\text { Mean RMS } \\
\text { errors, \% of } \\
\text { max. }\end{array}$ & $12 \pm 5$ & $14 \pm 6$ & $14 \pm 5$ & $18 \pm 8$ \\
\hline $\begin{array}{l}\text { Mean } \\
\text { maximal } \\
\text { joint } \\
\text { excursion } \\
\text { error, } \\
\text { degrees }\end{array}$ & $22.08 \pm 23.65$ & $26.31 \pm 14.54$ & $3.76 \pm 16.05$ & $6.27 \pm 14.77$ \\
\hline $\begin{array}{l}\text { Mean } \\
\text { maximal } \\
\text { joint } \\
\text { excursion } \\
\text { error, \% of } \\
\text { max. }\end{array}$ & $12 \pm 13$ & $14 \pm 8$ & $2 \pm 10$ & $7 \pm 16$ \\
\hline
\end{tabular}

Table contains mean values \pm standard deviations across participants and movements. Max. stands for maximal range of motion. 


\section{Acknowledgments}

The authors would like to thank Dr. Laurie Gutmann, Martha Power, and Dr. Christopher Cummings for their help in participant recruitment and Christopher Bauer and Aric Logsdon for their help with data collection.

\section{References}

1. Kelly-Hayes M, Beiser A, Kase CS, Scaramucci A, D'Agostino RB, et al. (2003) The influence of gender and age on disability following ischemic stroke: the Framingham study. J Stroke Cerebrovasc Dis 12: 119-126. doi:10.1016/S10523057(03)00042-9.

2. Go AS, Roger VL, Benjamin EJ, Berry JD, Borden WB, et al. (2013) Heart disease and stroke statistics - 2013 update: a report from the American Heart Association. Circulation. 240 pp. doi:10.1161/CIR.0b013e3182009701.

3. Desrosiers J, Noreau L, Rochette A, Bourbonnais D, Bravo G, et al. (2006) Predictors of long-term participation after stroke. Disabil Rehabil 28: 221-230. doi:10.1080/09638280500158372.

4. Morris JH, van Wijck F, Joice S, Donaghy M (2013) Predicting health related quality of life 6 months after stroke: the role of anxiety and upper limb dysfunction. Disabil Rehabil 35: 291-299. doi:10.3109/09638288.2012.691942.

5. Volpe BT, Lynch D, Rykman-Berland A, Ferraro M, Galgano M, et al. (2008) Intensive sensorimotor arm training mediated by therapist or robot improves hemiparesis in patients with chronic stroke. Neurorehab Neural Re 22: 305-310. doi:10.1177/1545968307311102.

6. Taub E, Wolf S (1997) Constraint induction techniques to facilitate upper extremity use in stroke patients. Top Stroke Rehabil 3: 38-61.

7. Duncan P, Studenski S, Richards L, Gollub S, Lai S, et al. (2003) Randomized clinical trial of therapeutic exercise in subacute stroke. Stroke 34: 2173-2180.

8. Wolf S, Lecraw D, Barton L, Jann B (1989) Forced use of hemiplegic upper extremities to reverse the effect of learned nonuse among chronic stroke and headinjures patients. Exp Neurol 104: 125-132.

9. Taub E, Miller N, Novack T, Cook E, Fleming W, et al. (1993) Technique to improve chronic motor deficit after stroke. Arch Phys Med Rehabil 74: 347-354.

10. Gritsenko V, Prochazka A (2004) A functional electric stimulation-assisted exercise therapy system for hemiplegic hand function. Arch Phys Med Rehabil 85: 881-885. doi:10.1016/j.apmr.2003.08.094.

11. Rubin MN, Wellik KE, Channer DD, Demaerschalk BM (2013) Systematic review of telestroke for post-stroke care and rehabilitation. Curr Atheroscler Rep 15: 343. doi:10.1007/s11883-013-0343-7.

12. Perry JC, Ruiz-Ruano JA, Keller T (2011) Telerehabilitation: toward a costefficient platform for post-stroke neurorehabilitation. IEEE Int Conf Rehabil Robot 2011: 5975413. doi:10.1109/ICORR.2011.5975413.

13. Huijgen BC, Vollenbroek-Hutten MM, Zampolini M, Opisso E, Bernabeu M, et al. (2008) Feasibility of a home-based telerehabilitation system compared to usual 
care: arm/hand function in patients with stroke, traumatic brain injury and multiple sclerosis. Journal of Telemedicine and Telecare 14: 249-256.

doi:10.1258/jtt.2008.080104.

14. Lai JCK, Woo J, Hui E, Chan WM (2004) Telerehabilitation - a new model for community-based stroke rehabilitation. Journal of Telemedicine and Telecare 10: 199-205. doi:10.1258/1357633041424340.

15. Rodriguez-de-Pablo C, Perry JC, Cavallaro FI, Zabaleta H, Keller T (2012) Development of computer games for assessment and training in post-stroke arm telerehabilitation. Conference proceedings : Annual International Conference of the IEEE Engineering in Medicine and Biology Society IEEE Engineering in Medicine and Biology Society Conference 2012: 4571-4574. doi:10.1109/EMBC.2012.6346984.

16. Durfee W, Carey J, Nuckley D, Deng J (2009) Design and implementation of a home stroke telerehabilitation system. Conference proceedings : Annual

International Conference of the IEEE Engineering in Medicine and Biology Society IEEE Engineering in Medicine and Biology Society Conference 2009: 2422-2425. doi:10.1109/IEMBS.2009.5334951.

17. Brennan DM, Lum PS, Uswatte G, Taub E, Gilmore BM, et al. (2011) A telerehabilitation platform for home-based automated therapy of arm function. Conference proceedings : Annual International Conference of the IEEE Engineering in Medicine and Biology Society IEEE Engineering in Medicine and Biology Society Conference 2011: 1819-1822. doi:10.1109/IEMBS.2011.6090518.

18. Broeren J, Dixon M, Sunnerhagen KS, Rydmark M (2006) Rehabilitation after stroke using virtual reality, haptics (force feedback) and telemedicine. Stud Health Technol Inform 124: 51-56.

19. Langan J, Delave K, Phillips L, Pangilinan P, Brown SH (2013) Home-based telerehabilitation shows improved upper limb function in adults with chronic stroke: a pilot study. J Rehabil Med 45: 217-220. doi:10.2340/16501977-1115.

20. Johnson MJ, Shakya Y, Strachota E, Ahamed SI (2011) Low-cost monitoring of patients during unsupervised robot/computer assisted motivating stroke rehabilitation. Biomed Tech (Berl) 56: 5-9. doi:10.1515/BMT.2010.050.

21. Chumbler NR, Quigley P, Li X, Morey M, Rose D, et al. (2012) Effects of telerehabilitation on physical function and disability for stroke patients: a randomized, controlled trial. Stroke 43: 2168-2174.

doi:10.1161/STROKEAHA.111.646943.

22. Fugl-Meyer AR, Jaasko L, Leyman I, Olsson S, Steglind S (1975) The poststroke hemiplegic patient: a method for evaluation of physical performance. Scand J Rehabil Med 7: 13-31.

23. Lyle RC (1981) A performance test for assessment of upper limb function in physical rehabilitation treatment and research. Int J Rehabil Res 4: 483-492.

24. Duncan P, Propst M, Nelson S (1983) Reliability of the Fugl-Meyer Assessment of sensorimotor recovery following cerebrovascular accident. Phys Ther 63: 1606-1610.

25. van der Lee J, Beckerman H, Lankhorst G, Bouter L (2001) The responsiveness of the Action Research Arm test and the Fugl-Meyer Assessment scale in chronic stroke patients. J Rehabil Med 33: 110-113. 
26. Gladstone DJ, Danells CJ, Black SE (2002) The fugl-meyer assessment of motor recovery after stroke: a critical review of its measurement properties. Neurorehab Neural Re 16: 232-240.

27. Berglund K, Fugl-Meyer AR (1986) Upper extremity function in hemiplegia. A cross-validation study of two assessment methods. Scand J Rehabil Med 18: 155157.

28. De Weerdt W (1985) Measuring recovery of arm-hand function in stroke patients: a comparison of the Brunnstrom-Fugl-Meyer test and the Action Research Arm test. Physiotherapy Canada 37: 65-70.

29. Hsieh Y-W, Wu C-Y, Lin K-C, Chang Y-F, Chen C-L, et al. (2009)

Responsiveness and validity of three outcome measures of motor function after stroke rehabilitation. Stroke 40: 1386-1391. doi:10.1161/STROKEAHA.108.530584. 30. Zheng H, Black ND, Harris ND (2005) Position-sensing technologies for movement analysis in stroke rehabilitation. Med Biol Eng Comput 43: 413-420.

31. Stone EE, Skubic M (2011) Passive in-home measurement of stride-to-stride gait variability comparing vision and Kinect sensing. pp. 6491-6494. doi:10.1109/IEMBS.2011.6091602.

32. Clark RA, Bower KJ, Mentiplay BF, Paterson K, Pua Y-H (2013) Concurrent validity of the Microsoft Kinect for assessment of spatiotemporal gait variables. J Biomech. doi:10.1016/j.jbiomech.2013.08.011.

33. Clark RA, Pua Y-H, Fortin K, Ritchie C, Webster KE, et al. (2012) Validity of the Microsoft Kinect for assessment of postural control. Gait Posture 36: 372-377. doi:10.1016/j.gaitpost.2012.03.033.

34. Clark RA, Pua Y-H, Bryant AL, Hunt MA (2013) Validity of the Microsoft Kinect for providing lateral trunk lean feedback during gait retraining. Gait Posture. doi:10.1016/j.gaitpost.2013.03.029.

35. Lloréns R, Alcañiz M, Colomer C, Navarro MD (2012) Balance recovery through virtual stepping exercises using Kinect skeleton tracking: a follow-up study with chronic stroke patients. Stud Health Technol Inform 181: 108-112.

36. Chang Y-J, Chen S-F, Huang J-D (2011) A Kinect-based system for physical rehabilitation: A pilot study for young adults with motor disabilities. Research in Developmental Disabilities 32: 2566-2570. doi:10.1016/j.ridd.2011.07.002.

37. Lange B, Chang C-Y, Suma E, Newman B, Rizzo AS, et al. (2011) Development and evaluation of low cost game-based balance rehabilitation tool using the Microsoft Kinect sensor. pp. 1831-1834. doi:10.1109/IEMBS.2011.6090521.

38. Pastor I, Hayes HA, Bamberg SJM (2012) A feasibility study of an upper limb rehabilitation system using Kinect and computer games. Conference proceedings : Annual International Conference of the IEEE Engineering in Medicine and Biology Society IEEE Engineering in Medicine and Biology Society Conference 2012: 12861289. doi:10.1109/EMBC.2012.6346173.

39. Fisher RA (1925) Statistical Methods For Research Workers. Genesis Publishing Pvt Ltd. pp. 1-362

40. Gatsonis C, Sampson AR (1989) Multiple correlation: exact power and sample size calculations. Psychol Bull 106: 516-524. 
41. Maclellan MJ, Richards CL, Fung J, McFadyen BJ (2013) Use of segmental coordination analysis of non-paretic and paretic limbs during obstacle clearance in community dwelling persons post stroke. PM R. doi:10.1016/j.pmrj.2013.02.003. 42. van Kordelaar J, van Wegen EEH, Kwakkel G (2012) Unraveling the interaction between pathological upper limb synergies and compensatory trunk movements during reach-to-grasp after stroke: a cross-sectional study. Experimental Brain Research 221: 251-262. doi:10.1007/s00221-012-3169-6. 43. Duff M, Chen Y, Cheng L, Liu SM, Blake P, et al. (2012) Adaptive Mixed Reality Rehabilitation Improves Quality of Reaching Movements More Than Traditional Reaching Therapy Following Stroke. Neurorehab Neural Re.

doi:10.1177/1545968312465195

44. Caimmi M, Carda S, Giovanzana C, Maini ES, Sabatini AM, et al. (2008) Using kinematic analysis to evaluate constraint-induced movement therapy in chronic stroke patients. Neurorehab Neural Re 22: 31-39.

doi:10.1177/1545968307302923.

45. Lin K-C, Wu C-Y, Wei T-H, Lee C-Y, Liu J-S (2007) Effects of modified constraint-induced movement therapy on reach-to-grasp movements and functional performance after chronic stroke: a randomized controlled study. Clin Rehabil 21: 1075-1086. doi:10.1177/0269215507079843.

46. Kunkel A, Kopp B, Muller G, Villringer K, Villringer A, Taub E, Flor H. (1999) Constraint-induced movement therapy for motor recovery in chronic stroke patients. Arch Phys Med Rehabil 80: 624-628. 


\title{
Chapter 3
}

\section{Automated Assessment of the Wolf Motor Function Test}

\author{
Authors and Contributors \\ Erienne Olesh contributed to the design of the study, subject recruitment, data \\ collection, data analysis, and writing of the manuscript. Valeriya Gritsenko \\ contributed to the data analysis, and to writing of the manuscript.
}




\section{Introduction}

Annually, 800,000 individuals in the United States will suffer from a stroke. Of those, nearly half will have long-term motor impairment, causing stroke to be the leading contributor to adult disability in the U.S. and unfortunately, this number is projected to rise (American Heart Association, 2016). Recent advances in the acute treatment of stroke, such as clot dissolving drugs and mechanical removal procedures, have fortunately increased the survival rate from stroke. However, only a small percentage of patients are able to reach a medical facility within the 4-6 hour time window after symptom onset that would allow for these treatments to be beneficial (American heart Association, 2007; Wahl and Schwab, 2014). Once outside of this brief window, the only therapy that can alter the long-term outcome of the patient is rehabilitation.

Spontaneous recovery of motor function in stroke patients is heterogeneous, and is likely impacted by the size and location of the infarct (Dobkin, 2005). Roughly 80 percent of stroke patients will see the majority of recovery within their first 30 days after a stroke (Nakayama et al., 1994). During this time period, the tissue surrounding the stroke core, also referred to as the penumbra, can increase or decrease in size, affecting which tissue is converted to core infarct area, and which tissue will be salvaged (Stinear et al., 2013; Krakauer et al., 2012). Studies by Duncan and colleagues have shown that a patient's recovery at 6 months can be predicted from their functional ability at 30 days post stroke. Additionally, the 30 day prediction was shown to be a better correlate of long term movement recovery then a 5 day post stroke comparison (Duncan et al., 1992). Rehabilitation studies have also shown that aggressive therapy during the acute phase after a stroke can provide the best long-term outcomes for a patient (Feys et al., 1998). Given this, it is therefor likely that the initial month post stroke is the best window for the most aggressive forms of rehabilitation to take place. However, to determine which type of rehabilitation is providing the most benefit to the patient during this narrow window, there must be a reliable and descriptive way to assess a patient's motor impairment.

Physicians often gage a patient's motor ability on the individual's ability to perform activities of daily living (ADL). This categorization of motor function however, can be a misleading representation of a patient's ability as compensatory mechanisms are often quickly learned, allowing them to still function in their day to day life. For instance, a patient may re-learn to brush their teeth despite partial arm paresis by developing alternative movement strategies that accomplish the same task but in a manner that does not match typical movement. A physician may see the ability of their patient to brush their teeth and assign them a higher score on an ADL test, despite the fact that they are clearly impaired. To combat this, physical therapists and researchers have devised tests that measure overall movement impairment of a patient, rather than their ability to complete a given task. These tests, such as the Fugl-Meyer (Fugl-Meyer, 1975) and the Wolf Motor Function test (Wolf et al., 2001) provide a better picture of a patient's overall functional ability, rather than their ability to perform discrete tasks. Despite this improvement in post stroke movement assessment, these tests are timely to complete, require specialized 
equipment and must be administered by specifically trained individuals. Due to these constraints, such assessments are not often used in clinical practice.

A solution to this lack in quantitative measures could be the incorporation of motion capture into clinical assessments of movement impairment. By using motion capture in the clinical evaluation of a patient, information such as maximum range of motion, speed of movement, reachable workspace volume and other direct metrics of movement ability could be captured. This information would provide a more complete picture of movement capability and therefor could be used to track a patient's response to a given therapy program.

Improvements in motion capture technology are making the incorporation of motion capture technology into clinical practice a reality. Until recent years, the use of motion capture for clinical purposes was prohibitively expensive and required a skilled technician trained in motion capture equipment and analysis. However, new video game technology, such as the Microsoft Kinect, has alleviated the burden of costly motion capture by providing a low cost alternative. This technology has the ability to bring full body three dimensional motion capture into the clinical setting, allowing clinicians to truly measure their patients' motor function. The Kinect system is inexpensive, easy to use, and can be set up in a clinic, hospital, or home. Already, these systems have demonstrated usefulness in the assessment of balance deficits, the ability to automatically assess different parameters of gait, and have been successfully incorporated into multiple types of rehabilitation programs (Lange et al., 2011; Stone et al., 2011; Chang et al., 2011;).

To further test the capabilities of low cost motion capture and to address the need for a quantitative measure of post stroke movement ability, we proposed the development of a quantitative assessment of motor impairment that combines a low cost motion capture system and a computer algorithm. Together, this system would record a patient's movement, quantify their impairment and provide clinicians with a measure of motor function.

Previous research from our lab has demonstrated the feasibility of this system by comparing the quality of motion capture from a low cost system (the Microsoft Kinect) to an expensive state of the art system (PhaseSpace). We demonstrated that not only was the quality of the low-cost system comparable to the high-cost system, but that the assessment of a stroke patients movement was better than the reliability of several Doctoral of Physical Therapy students. During this initial experiment, stroke patients were asked to perform a range of motion tasks that mimicked clinical tests of movement impairment including the Fugl-Myer Assessment (FMA) and the Action Research Arm Test (ARAT). While patients performed these movements, simultaneously recordings from the PhaseSpace and Kinect systems tracked the each patient's movement (for further details see chapter 2, Automated Assessment of Movement Impairment Due to Stroke).

To elaborate on these initial findings, we choose to investigate whether the same automated system as tested in our previous experiment could accurately assess a stroke patient performing the Wolf Motor Function Test (WMFT). This test includes both complex and fine motor tasks, such as moving a basket, picking up paper clips, and lifting a can. These movements were more complex then the tasks previously studied and also involved patient interaction with objects. It was therefor 
our intent to determine how well the Microsoft Kinect could capture the complexity of movement and the interaction between patients and objects encompassed in the WMFT. Additionally, we aimed to compare the motion-capture based scores to scores assigned to movement by physical therapy doctoral students.

\section{Methods}

Eight chronic motor impaired stroke patients (defined as having movement impairment 3 months or more post stroke) were recruited from Ruby Memorial Hospital. Subjects were included in the study if they had suffered from an ischemic stroke and could produce voluntary movement of their more impaired limb. Patients were excluded if they suffered from any additional diseases or injuries that would affect movement ability (including but not limited to Multiple Sclerosis, Cerebral Palsy, shoulder repair or replacement surgery). Additionally, only patients that could independently sign informed consent were included. Stroke location was determined by MRI scan, read and confirmed by a clinical neurologist from Ruby Memorial Hospital.

To begin the test, each patient watched a pre-recorded video demonstrating one movement from the WMFT. The patient was then instructed to repeat the movement with either their unimpaired or impaired limb. This was repeated for all fifteen movements that make up the WMFT and limb order was randomized. Each task was performed only once as instructed by the WMFT. The Kinect sensor was placed two meters directly in front of the subject to optimize viewing capabilities. Video recordings of each movement were de-identified and given to four Physical Therapy Doctoral students at West Virginia University to score on the WMFT scale of $0-5(0=$ no movement, $5=$ perfect movement $)$.

Motion capture recorded by the Kinect consisted of three-dimensional coordinate data from ten tracked points on the upper-extremities and trunk. These data were imported into Matlab (MathWorks) and used to calculate the following joint angles: shoulder flexion/extension, shoulder abduction/adduction, shoulder pronation/supination, elbow flexion/ extension, wrist flexion/extension, and wrist pronation/supination. From those joint angles, quantitative scores were calculated using the same method as described in Olesh et al., 2013. In brief, Principal Component Analysis (PCA) was applied to the data set recorded for each movement performed by the less impaired limb of each patient. The extracted components were then used to reconstruct data for both the less and more impaired limb. Movements reconstructed for the more impaired limb were then compared to the original data from the more impaired limb using the square of the Pearson's Correlation Coefficient. This calculated $\mathrm{R}^{2}$ value was deemed as the quantitative score, assigned by the algorithm. By comparing the original data from the more impaired limb to the reconstructed data we were able to quantify the difference in movement capabilities of the two limbs.

\section{Results and Conclusions}

Mean quantitative scores, for each subject obtained from the algorithm, were compared to the mean qualitative scores assigned by the human raters (Fig. 1). 
Despite our previous success with this algorithm, results from this study did not reach statistical significance.

We have speculated several reasons for why the automated scoring system did not perform to an adequate standard in this experiment. First, the movements that make up the WMFT include several fine motor tasks (flipping cards, stacking checkers, picking up a paper clip, etc.) which are likely difficult for the Kinect to track. The version of the Kinect sensor used during this project has only a single point to represent the orientation of the hand. For proper biomechanical reconstruction of movement, three points on a plane are needed.

Second, the WMFT requires patients to be seated at a table for almost all of the movements, which restricts the Kinect sensors viewing angle. This could have also contributed to poor kinematic data quality as the Kinect sensor was initially designed to recognize standing, not seated, postures. Although the Kinect sensor and automated scoring method did not produce reliable results in this experiment, it should not be disregarded as a possible rehabilitation tool. A new version of the Kinect sensor was released in 2014 that demonstrates improved kinematics as well as increased detail of hand posture. This constantly developing technology will likely provide the much needed improvements to standard clinical rehabilitation in the future.

Figure 1. Comparison of quantitative to qualitative scores. Movements were scored on a 0 to 5 scale, as directed by the WMFT. Qualitative scores were calculated as the average score assigned by the eight physical therapy students. Quantitative scores were calculated from the PCA based scoring algorithm (see methods). Scores were compared using regression analysis. 


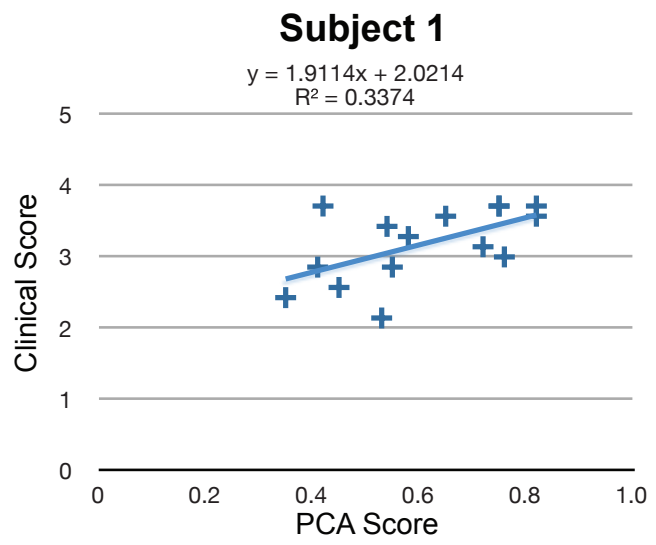

Subject 3

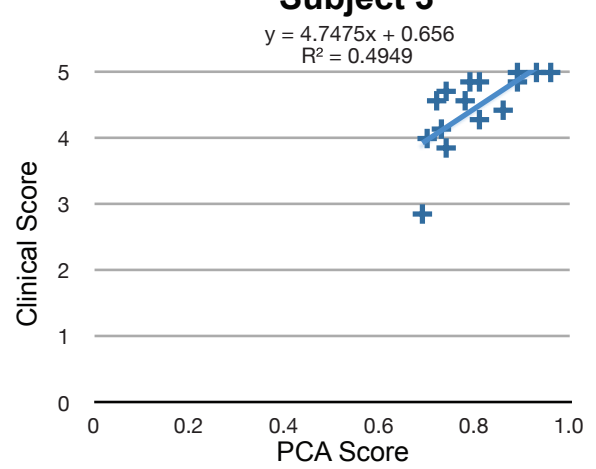

Subject 5

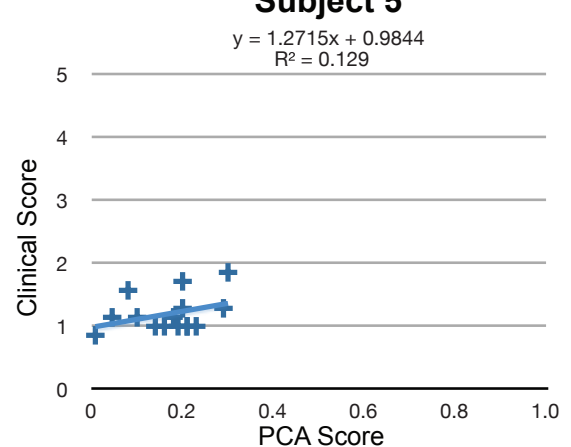

Subject 7

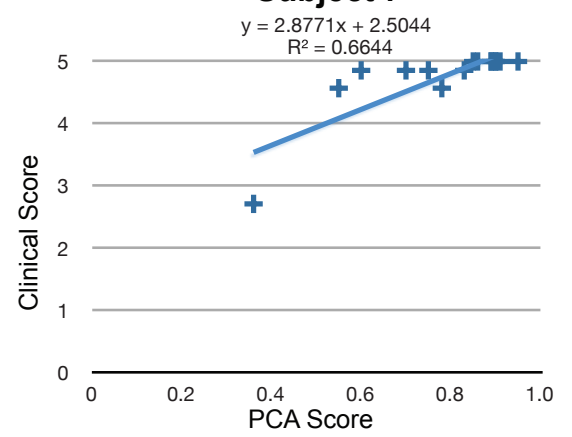

Subject 2

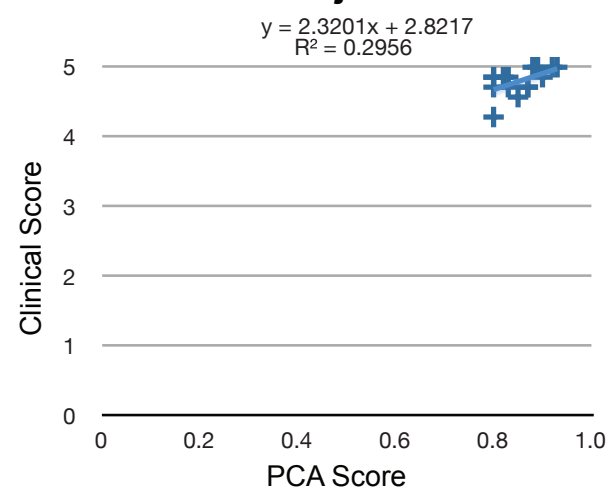

Subject 4

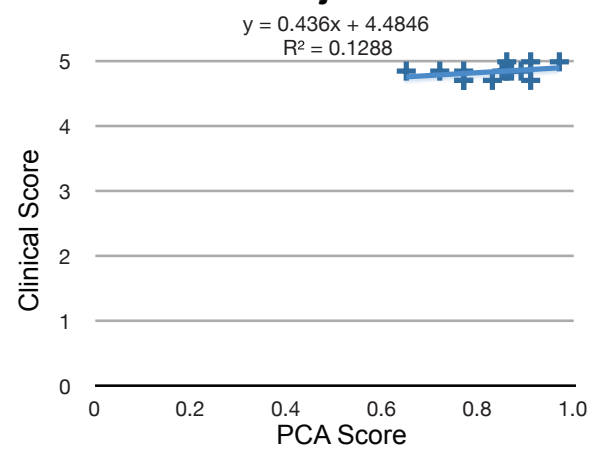

Subject 6

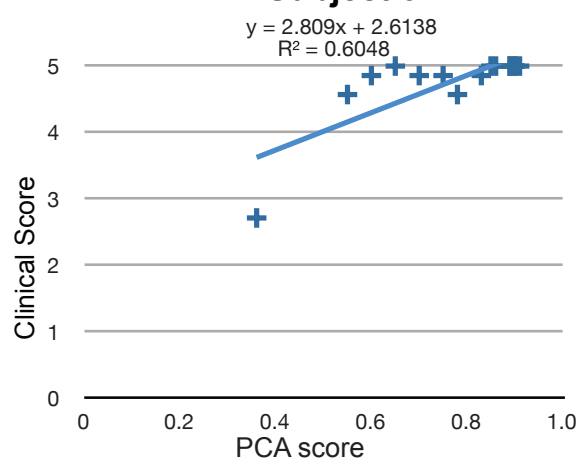

Subject 8

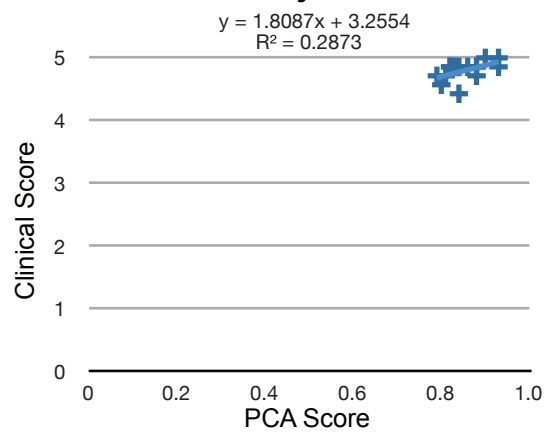




\section{Chapter 5}

Gravitational signals underlie tonic muscle activity during goal-directed reaching

Abbreviated title: gravitational motor primitives

\section{Authors and affiliations:}

Erienne Olesh ${ }^{1,3}$, Bradley Pollard1,3, and Valeriya Gritsenko1,2,3,*

${ }^{1}$ Department of Human Performance, School of Medicine, West Virginia University, Morgantown, West Virginia, United States of America

2 Department of Mechanical and Aerospace Engineering, Benjamin M. Statler College of Engineering and Mineral Resources, West Virginia University, Morgantown, West Virginia, United States of America

${ }^{3}$ Blanchette Rockefeller Neurosciences Institute, West Virginia University School of Medicine, Morgantown, West Virginia, United States of America

\section{Correspondence:}

Valeriya Gritsenko

vgritsenko@hsc.wvu.edu 


\section{Abstract}

Human reaching movements require complex muscle activations to produce the forces necessary to move the limb in a controlled manner. How the complex kinetic properties of the limb and gravity contribute to the generation of the muscle activation pattern by the central nervous system (CNS) is a long-standing question in neuroscience. One common theory is that the CNS reduces the redundancies and complexities of the musculoskeletal system using motor primitives. These primitives are often obtained using decomposition methods based on shared variance across multiple signals. A critique of this technique is that the dependencies that exist due to the causal relationship between muscle activations and the resulting movement are difficult to disambiguate from neural primitives inherent in control signals. In the present study addressed this critique by examining the relationships between motor primitives extracted from muscle activity, muscle torques, and other motion signals. We hypothesized that the primitives obtained from muscle activity are more similar to kinetic primitives obtained from joint torques, than kinematic primitives obtained from joint angles and angular velocity signals. Eight healthy subjects pointed in virtual reality to visual targets arranged to create a standard center-out reaching task in three dimensions. Muscle activity and motion capture data were synchronously collected during the movements. Non-negative matrix factorization was then applied to muscle activity, muscle torques, and other motion signals (joint angles, angular velocities, gravitational torques, and other inertial torques) separately to reduce the dimensionality of data. Results show that the activation profiles of all NMF components were organized sequentially and correlated highly. The scaling of NMF components obtained from EMG and kinetic and kinematic signals correlated across multiple signal types. We found closer correspondence between NMF components obtained from EMG and gravitational torques, than those obtained from other torque signals or kinematic signals. Altogether, these results reject our hypothesis, suggesting that motor primitives do not consist of signals of a single modality. Our results also identify the kinetic signals for gravity compensation as the potential contributor to neural motor primitives that may be responsible for controlled transitions between arm postures during movement.

\section{Introduction}

The musculoskeletal anatomy of the body constitutes a complex dynamical system that is a challenge to control for the central nervous system (CNS). Some of the complexity is due to muscle redundancy that allows humans to perform complex tasks. Additional complexity is due to the forces associated with the inertia of the multi-joint limb, termed limb dynamics, which must also be accounted for by the CNS. Limb dynamics is commonly investigated through joint torques, or rotational forces, that arise during motion of the limb (Sainburg et al., 1995; 1999; Shabbott and Sainburg, 2008). This motion, commonly expressed as angular kinematics (position and velocity), can be used to derive joint torques for each independent direction of motion termed degree of freedom (DOF) using equations of motion. The goal is to derive the active torques that are generated because of muscle 
contractions in the presence of passive forces, such as those due to gravity or the interaction between connected segments (Dounskaia and Wang, 2014; Gentili et al., 2007; Le Seac'h and McIntyre, 2007; Papaxanthis et al., 2005). The latter passive interaction torques comprise a sizable amount of the overall torques experienced during arm movement (Hollerbach and Flash, 1982). The compensation for interaction torques appears to be an important factor for the neural control of goaldirected movement (Debicki and Gribble, 2005; Gribble and Ostry, 1999; Gritsenko et al., 2009; 2011; Pigeon et al., 2003). Other passive torques arise due to gravity; these gravitational torques depend on the orientation of limb segments in space (Bastian et al., 1996). The compensation for gravitational torques is also important for motor control, as evidenced by altered patterns of movement errors and muscle activity of people moving in micro-gravity environments (Fisk et al., 1993;

Papaxanthis et al., 1998; 2005; Pozzo et al., 1998). Altogether, the action of the CNS to control these passive torques can be observed at least partially through analysis of active muscle torques, which are the summed result of muscle contraction. Thus, muscle torques are a window into the interaction between the CNS and the musculoskeletal anatomy of the limb.

A prevalent explanation of how the CNS resolves the complexity of limb motor control is based on the idea of motor primitives, i.e. groups of muscles sharing the same common source of neural activation (Mussa Ivaldi, 1999; Mussa Ivaldi and Bizzi, 2000). These are usually extracted using decomposition methods, such as principle component analysis (PCA), or non-negative matrix factorization (NMF) (Ting, 2007; Tresch et al., 2006). These motor primitives have been shown to be most active for movements and in response to perturbations in specific directions, i.e. directionally tuned (d'Avella et al., 2006; Torres-Oviedo and Ting, 2007). They can also be scaled in both time and amplitude to adjust for changing speeds and distances during movement (d'Avella et al., 2008). It has also been suggested that motor primitives may be structured in such a way as to compensate for task-specific limb dynamics (Chvatal et al., 2011). Central to this concept is the idea that motor primitives can reduce the complexity of neural control signals by enabling the production of any movement from a smaller selection of control actions (Bizzi et al., 1991; Giszter et al., 1993) for a review see (d'Avella and Lacquaniti, 2013).

However, the method of obtaining motor primitives using decomposition analyses has recently come under increased scrutiny due to the indivisible interaction and mutual dependencies between the neural control of muscular activations and the biomechanics of the resulting movement (Santello et al., 2013; Tresch and Jarc, 2009). For example, multiple independent DOFs of hand joints are known to be mechanically coupled through tendons that span several joints of a finger and the wrist. This reduces the overall range of possible motions to a much smaller subset of kinematic primitives, which could contribute to the motor primitives identified in muscle activations using decomposition methods (Valero-Cuevas et al., 2015). Furthermore, primitives obtained from joint kinematics and muscle activations were found to be mutually dependent (Kutch and Valero-Cuevas, 2012; Tagliabue et al., 2015). The latter study also shows that kinematic constraints can create the appearance of muscle primitives in simulated data, in which no neural primitives are expected a-priori. 
In the current study, we further examine the transformation from muscle activation to movement to establish the role that motion kinematics and limb dynamics play in the generation of neural motor commands to muscles. The rationale for our approach is based on the examination of shared variance with NMF decomposition method across different types of motion-related signals. Among kinematic and kinetic signals, muscle activations are most closely related to kinetic signals such as active muscle torques described above. The muscle torques can in turn be split into two components, 1) gravitational torques that only arise in the presence of gravity and 2) other inertial torques, including interaction torques, that are responsible for inter-joint coordination. Each of these components can be calculated for each joint DOF. However, across multiple joint DOFs, signals for each of these components are coupled through the kinematic chain of the limb. This coupling can be quantified using NMF to obtain kinetic primitives, which can then be compared to the primitives obtained from muscle activations. We hypothesize that primitives obtained from muscle activations are more similar to kinetic primitives obtained from torque components, than kinematic primitives obtained from joint angles and angular velocity signals. Support for this hypothesis would suggest that kinetic signals for gravity compensation and inter-joint coordination may comprise neural motor primitives.

\section{Material and Methods}

Eight healthy individuals ( 5 males, 3 females) with an average age of $24.8 \pm 0.71$ years old were recruited to perform a reaching "center-out" task. This study was carried out in accordance with the recommendations the Institutional Review Board of West Virginia University with written informed consent from all subjects. All subjects gave written informed consent in accordance with the Declaration of Helsinki. The protocol was approved by the Institutional Review Board of West Virginia University (Protocol \# 1311129283). All subjects were right-hand dominant and reported no movement disorders and no major injuries to their right arm. Height, weight, and arm segment lengths were measured for each subject and used to adjust model parameters to create subject-specific dynamic models (see below).

Movements were instrumented using a virtual reality software (Vizard by Wolrdviz) and head set (Oculus Rift), which displayed 14 targets arranged in two perpendicular planes: the horizontal transverse plane and the vertical coronal plane (Fig. 1A). To reduce inter-subject variability in kinematic data, the target locations were adjusted for each subject based on the lengths of their arm segments, which ensured the same initial and final joint angles across all movement directions. The center target was placed so that initial arm posture was at 0 -degree shoulder flexion, 90-degree elbow flexion, and a 0-degree wrist flexion. The distance from the center target to the peripheral targets was scaled to 30 percent of each subject's total arm length (from anterior acromial point to the distal end of the index finger). Each movement began with the subject starting at the center target and then moving to another visible target cued by target color change. Subjects were instructed to keep their wrist pronated and straight and move as quickly and 
accurately as possible. Movements to each target location were repeated 15 times and performed in a randomized order.

Fig. 1 near here

Arm and trunk movements were recorded with an active motion capture system (PhaseSpace, Impulse) at 480 frames per second. The light emitting diodes of the motion capture system were placed on anatomical landmarks according to best practice guidelines (Robertson et al., 2004). Electromyography (EMG) was recorded from twelve arm muscles at a rate of $2000 \mathrm{~Hz}$ (MA400-28 MotionLab Systems). Muscles recorded during the experiment included the pectoralis major (Pec), teres major (TrM), anterior deltoid (AD), posterior deltoid (PD), long and short heads of the biceps (BiL and BiS respectively), lateral and long heads of the triceps (TrLa and TrLo respectively), brachioradialis (Br), flexor carpi ulnaris (FCU), flexor carpi radialis (FCR), and extensor carpi radialis (ECR). Motion capture and electromyography were synchronized using a custom circuit and triggering mechanism (Talkington et al., 2015). Motion capture and EMG data were imported into Matlab and processed as follows using custom scripts.

EMG data were high pass filtered at $40 \mathrm{~Hz}$, bandpass filtered between 59 and $61 \mathrm{~Hz}$ to remove electrical background noise, rectified, and low pass filtered at $20 \mathrm{~Hz}$. Motion capture data were low pass filtered at $10 \mathrm{~Hz}$ and interpolated with a cubicspline. The maximum interpolated gap was 0.2 seconds. The onset and offset of movement was found based on the velocity of three hand LEDs changing by five percent of the maximum velocity for a given movement. Arm kinematics were obtained from motion capture by calculating Euler angles and angular velocity for five joint DOFs including shoulder (flexion/extension, abduction/adduction, pronation/supination), elbow (flexion/extension), and wrist (flexion/extension).

\subsection{Limb dynamics}

To calculate joint torques, an inverse dynamic model of the subject's arm was constructed in Simulink (MathWorks). The model comprised 5 DOFs as described above and three segments approximating inertial properties of the arm, forearm, and hand. Inertia of the segments was approximated with a cylinder of the length equal to that of the corresponding segment and a $3-\mathrm{cm}$ radius. The masses and centers of mass for each segment were determined by their anthropometric ratios to the subjects' segment lengths and weight (Winter, 2009).

Angular kinematics averaged per movement direction and per subject was used in the subject-specific inverse model to calculate joint torques (Fig. 1B). These computed torques are proportional to the sum of all moments generated by muscles spanning the joints, so these torques are referred to as muscle torques in the rest of the manuscript. The numerical quality of inverse dynamic simulations was checked by running the same model in forward dynamics mode using the calculated torques as inputs and simulated angular kinematics as outputs. The simulated and experimental joint kinematics was compared, and the mean \pm standard deviation of 
the root-mean-squared differences between them was $0.05 \pm 0.02$ radians across all DOFs.

To test the main hypothesis, the muscle torques obtained using the inverse model were separated into two components. To estimate the component of muscle torques responsible for inter-joint coordination without gravity, the inverse model was run without simulating external gravitational force. This resulted in muscle torques that would produce the same motion without gravity as that recorded in presence of gravity. Example of such torques would be the sum of muscle moments produced during motion in microgravity environment. Then the component of muscle torque that is needed to compensate for gravity was estimated as the difference between muscle torques with and without gravity as follows:

$\tau_{M G}=\tau_{M}-\tau_{M G 0}$

where $\tau_{\mathrm{G}}$ is a vector of torques that only arise in the presence of gravity; $\tau_{\mathrm{m}}$ is a vector of muscle torques around each DOF during simulations with gravity; $\tau_{\text {мсо }}$ is a vector of computed torques around each DOF during simulations without gravity. If we assume that the torques produced without gravity are equal to the component of the motor command that is responsible for inter-joint coordination only, without gravity, then formula (1) can be rearranged as follows:

$\tau_{M N}=\tau_{M}-\tau_{M G}$, or

$\tau_{M}=\tau_{M N}+\tau_{M G}$

where $\tau_{\mathrm{m}}$ is the component of muscle torque responsible for inter-joint coordination, i.e. interaction torques and other inertial torques excluding gravity, and $\tau_{\mathrm{MG}}$ is the component of muscle torque responsible for the compensation for all torques due to gravity. Below, the former is referred to as MN torque, while the latter is referred to MG torque for simplicity.

\subsection{Motor Primitive Decomposition}

Motor primitives were extracted for each subject from EMG, kinematic and dynamic data separately using NMF (Berniker et al., 2009; Torres-Oviedo et al., 2006). To extract EMG primitives (NMF1 in Fig. 1B), rectified EMG signals were normalized to movement duration, averaged per movement direction, and low pass filtered at 10 Hz. To ensure muscle activations were unitless, maximum contraction values were calculated for each muscle across all movement directions and used to divide mean EMG for each movement direction. The resulting data matrix was comprised of 336 columns (12 EMG signals for 14 movements toward each virtual target and 14 return movements). Using the NMF algorithm described in Tresch et al. (Tresch et al., 1999), EMG primitives were extracted for each subject.

$m(t)=\sum_{i=1}^{N} C_{i} W_{i}(t)$ 
where $m(t)$ is the EMG matrix of average activity of all muscles during all movements at time $t ; N$ is the number of primitives; $C_{i}$ is the array of weights for primitive $i$ for each muscle and movement; and $W_{i}(t)$ is the activation of primitive $i$ at time $t$ (Fig. 2). The number of EMG primitives was increased until the variance accounted for (VAF) in EMG reached 95\%.

Fig. 2 near here

To extract muscle-torque primitives, a data matrix was constructed for each subject that included muscle torques for each DOF and each movement direction (NMF2 in Fig. 1B). The signals were rectified then normalized to the largest value of the signal for each DOF across all movement directions. The resulting data matrix was comprised of 120 columns ( 5 muscle torque signals for the 28 movement directions). NMF was applied to this data with the same criteria described above.

$\tau_{M}(t)=\sum_{i=1}^{N} A_{i} Y_{i}(t)$

where $\tau_{M}(t)$ is the muscle torque matrix for all signals per DOF per movement direction; $N$ is the number of primitives; $A_{i}$ is the weight matrix for primitive $i$ for each DOF and movement; and $Y_{i}(t)$ is the activation profile of the corresponding primitive $i$.

To extract mechanical primitives, a data matrix was constructed that included joint angles, angular velocity, MG torque and MN torque for each DOF (NMF3 in Fig. 1B). The signals were averaged across the fifteen repetitions of each movement direction. The same rectification, and normalization procedures were applied to the signals as described above for muscle torques. This ensured the same scale and unit independence across all signals. The data matrix comprised 560 columns $(20$ kinematic and kinetic signals for the 28 movement directions). NMF was applied to this data with the same criteria described above.

$d(t)=\sum_{i=1}^{N} B_{i} X_{i}(t)$

where $d(t)$ is the matrix of average profiles for all signals; $N$ is the number of primitives; $B_{i}$ is the weight matrix for primitive $i$ for each signal; and $X_{i}(t)$ is the activation profile of the corresponding primitive $i$.

The rectification procedure changed the profiles of the muscle and inertial torques, which could affect the comparison between motor primitives based on these signals and EMG. Rectification of inertial torques poses less of a problem, because these signals contain two readily identifiable phases of acceleration and deceleration that match the actions of individual muscles. Rectification of these signals results in profiles with two burst-like shapes that correspond to the timing of the two phases. However, rectification of muscle torques results in more variable changes in their profiles that are not easily linked to specific movement phases. To address this concern, we tested the validity of NMF on the rectified torque signals to accurately capture the relationship between signals. For this we substituted equations (4) and 
(5) into equation (2). Given that our analysis, described below, found highly correlated NMF temporal profiles across signals (Fig. 4), the resulting equation can be reduced as follows:

$A_{i}=B_{i 1}+B_{i 2}$

where for primitive $i$, NMF weight $A$ for each muscle torque signal is the sum of NMF weights $B_{1}$ and $B_{2}$ for $\mathrm{MN}$ and $\mathrm{MG}$ torques respectively for corresponding DOFs. The NMF weights calculated from rectified muscle torques differed from those calculated using equation (6) between $1 \pm 4 \%$ and $3 \pm 6 \%$ across subjects. The threshold for VAF was 95\%, which puts the differences in weights between the two methods below this threshold. This shows that NMF on rectified muscle torque signals captures accurately the linear relationships between those and other signals. Resulting activations $W_{i}(t), X_{i}(t)$, and, $Y_{i}(t)$ were normalized from zero to one for each subject. Normalization values were obtained by calculating the peak value from each subject's activation. For comparisons across signal types and across subjects, activations $W_{i}(t), X_{i}(t)$, and, $Y_{i}(t)$ were sorted per the relative timing of their maximal peak and assigned a letter in alphabetical order (Fig. 2A).

To maintain the relative relationship between the weights and activations, NMF weights, $C_{i}, A_{i}$, and $B_{i}$ were multiplied by the normalization value of the corresponding activation. This ensured that the variance for each movement direction was now captured by the NMF weights, not activations. Thus, NMF weights were then used to compare directional tuning between EMG, muscle-torque, and mechanical primitives. Coefficient of determination $\left(\mathrm{r}^{2}\right)$ was used as measures of similarity between the NMF weights obtained from different signals, NMF1, NMF2, and NMF3. Note that signals used for all NMF analyses were unitless. Correlation matrices were calculated between NMF weights $C_{i}$ and $A_{i}$, and between NMF weights $C_{i}$ and $B_{i}$ across corresponding directions of movement for first and last primitive only.

\subsection{Statistical analysis}

The statistical analysis of $r^{2}$ values was done using repeated measures analysis of variance (rANOVA) in MATLAB. Separate rANOVA tests were applied to $r^{2}$ values calculated between EMG and muscle-torque primitives (rANOVA1: weights from NMF1 vs. NMF2) and between EMG and mechanical primitives (rANOVA2: weights from NMF1 vs. NMF3). rANOVA1 included 2 factors, Joint and Primitive factor. The Joint factor grouped $\mathrm{r}^{2}$ values based on the joint the signals spanned, comprising 3 levels for shoulder, elbow, and wrist. The Primitive factor grouped $\mathrm{r}^{2}$ values based on the timing of the activation profile of each primitive, comprising 2 levels for the first primitive $A$ and the last primitive $\mathrm{C}$ or E (Fig. 4). rANOVA2 included 3 factors, Joint and Primitive factors as in rANOVA1 and a Signal factor. The Signal factor grouped $r^{2}$ values based on the types of signals used in NMF3, comprising 4 levels for joint angles, angular velocity, MG torque, and MN torque. Post-hoc multiple comparisons were used to further examine significant interactions. 
Cross-correlation analysis was used to compare activations $W_{i}(t), X_{i}(t)$, and, $Y_{i}(t)$. The temporal shifts that produced the highest correlations between each pair of activations were converted into the time domain from the normalized number of samples by using the mean duration of movement per subject. Positive lag times indicate that the second signal follows the first in each pair. A negative lag time indicates that the second signal precedes the first.

The relationships between peaks of activation for subsequent primitives obtained with NMF1, and between peaks of activation for corresponding primitives obtained with NMF1 vs. NMF2 and NMF3 were quantified using a linear regression. The same peaks of each activation used for the classification of the primitives (Fig. 2A) were used in this regression analysis.

\section{Results}

The angular kinematics of pointing to targets in virtual reality was highly consistent, as demonstrated by the low standard deviations of angular kinematics across the fifteen repetitions of each movement (Fig. 3A). The peak velocity of these movements ranged from 1.2 to 3.3 meters per second, which illustrates subjects' preferred speeds in response to instructions to point as quickly and accurately as possible (Fig. 3B). The consistent kinematics are attributed to the very consistent muscle torques, whose temporal profiles varied little across subjects (Fig. 3C). However, muscle activity was highly consistent within subjects (Fig. 3F), but varied between subjects.

Fig. 3 near here.

Muscle torques were divided into gravitational and other inertial torques (termed MG and MN torques) as described above. Gravitational torques were consistent within and across subjects and showed similar temporal profiles to joint angles (Fig. 3D). Inertial torques were also consistent within and across subjects. MN torques had activation profiles that were distinct from MG torque profiles (Fig. 3E) and similar to acceleration profiles derived from angular kinematics. In a given movement, MG torques tended to vary in a single direction increasing or decreasing in amplitude, while the MN torques usually comprised acceleration and deceleration phases characteristic of a bell-shaped velocity profile. For multiple movements, torques of the same type were coupled across DOFs (Figs 3D and 3E).

NMF1 on EMGs showed that between 2 and 5 EMG primitives were necessary to reach a $\mathrm{VAF}=96.6 \pm 0.9 \%$. The primitives were labeled A through $\mathrm{E}$, corresponding to the order in which the peak of the activation profile occurred. The activation profile of primitive A peaked at $1.0 \pm 2.1 \%$ of the average movement duration, followed by primitive B at $16.7 \pm 14.0 \%$, primitive $\mathrm{C}$ at $18.5 \pm 19.8 \%$, primitive $\mathrm{D}$ at $28.6 \pm 13.6 \%$, and primitive $\mathrm{E}$ at $89.0 \pm 11.0 \%$ (Fig. 4, red lines).

Fig. 4 near here 
NMF2 on muscle torques showed that, unlike NMF1, only 3 muscle-torque primitives were required to reach a VAF $=96.4 \pm 0.0 \%$ across subjects. Primitives were labeled as $\mathrm{A}$ through $\mathrm{C}$ based on their temporal sequence as described above. Activation profiles of primitive A peaked at $2.1 \pm 4.5 \%$ of the average movement duration, followed by primitive B at $35.7 \pm 15.7 \%$, and primitive $\mathrm{C}$ at $99.7 \pm 1.0 \%$ (Fig. 4, blue lines).

NMF3 on mechanical signals showed that 3 mechanical primitives were required to reach a VAF $=96.8 \pm 0.3 \%$ across subjects, same as the number derived by NMF2. Mechanical primitives were labeled as described above; the activation of primitive $A$ peaked at $0.3 \pm 0.0 \%$ of the average movement duration, followed by primitive $\mathrm{B}$ at $42.2 \pm 17.3 \%$, and primitive $\mathrm{C}$ at $98.5 \pm 3.3 \%$ (Fig. 4 , black lines).

The activation profiles obtained with NFM represent the amount of recruitment of a given primitive and may be more closely related to the temporal evolution of neural commands. The activation profiles were very similar not only across subjects, but also across signal types. The activation of sequential EMG primitives was highly correlated with peak correlation coefficients across subjects ranging from $0.84 \pm$ 0.07 between the first and second EMG primitive to $0.56 \pm 0.11$ between the first and last EMG primitive. These peak correlations occurred at lag times that were increasing on average at $170 \mathrm{~ms}$ intervals (Fig. 5A). The activation profiles of EMG primitives peaked at times that were linearly increasing $\left(r^{2}=0.94 ;\right.$ Fig. 5B). Furthermore, the activations of overlapping EMG and muscle-torque primitives were highly correlated with peak correlation coefficients across subjects ranging from $0.94 \pm 0.03$ between the first EMG and first muscle-torque primitive to $0.53 \pm$ 0.05 between the first EMG and last muscle-torque primitive. These peak correlations occurred at incremental lag times (Fig. 5C). The activation profiles of EMG primitives peaked at times that were linearly related to the peaks in the corresponding activations of muscle-torque primitives $\left(r^{2}=0.97\right.$; Fig. 5D). Similar relationships were observed between the activations of EMG and mechanical primitives (Figs $5 \mathrm{E}$ and $5 \mathrm{~F} ; \mathrm{r}^{2}=0.96$ ). This shows that the activation profiles of all primitives were organized sequentially and correlated highly.

Fig. 5 near here

\subsection{The overlap in directional tuning between different types of primitives}

To determine if EMG primitives have the same directional tuning as muscle-torque primitives, shared variance $\left(\mathrm{r}^{2}\right)$ between weights from NMF1 and NMF2 across all movement directions was calculated for the first and last primitive for each subject. Separate comparison for each primitive ensured that the temporal distribution of the primitives obtained from EMG and muscle-torques were matched (Fig. 4). The $\mathrm{r}^{2}$ values between NMF weights were small for most signal combinations, which indicates little overlap between directional tunings of EMG primitives and muscletorque primitives (Fig. 6). There were some differences in $r^{2}$ values of both primitives for signals originating from the different joints, but post-hoc comparisons revealed no consistent effects (Table 1). 
Fig. 6 and Table 2 near here

To determine if EMG primitives have the same directional tuning as mechanical primitives, shared variance $\left(\mathrm{r}^{2}\right)$ between weights from NMF1 and NMF3 across all movement directions was calculated for the first and last primitive for each subject. Separate comparison for each primitive ensured that the temporal distribution of the primitives obtained from EMG and kinematic and kinetic signals are matched (Fig. 4). The $r^{2}$ values between NMF weights were larger than in previous analysis for many signal combinations (Fig. 7). In the beginning of movement, the mean $\mathrm{r}^{2}$ for MG and MN torque signals was larger than the mean $r^{2}$ for velocity and angle signals for corresponding DOFs in 9 (out of 12) muscles, while the opposite was true for 1 muscle (TrLa) and no change was observed in 2 muscles (BiS and TrLo; Fig. 7, Primitive A). At the end of movement, the mean $\mathrm{r}^{2}$ for $\mathrm{MG}$ and $\mathrm{MN}$ torque signals was larger than the mean $r^{2}$ for velocity and angle signals for corresponding DOFs in only 5 muscles (FCU, BiL, BiS, TriLA, and AD), while the opposite was true in 5 other muscles (FCR, ECR, Br, TrLo, and Pec) and no change was observed in 2 muscles (TrM and PD; Fig. 7, Primitive C/E). Larger $r^{2}$ values were observed between NMF weights from EMG signals and MG torques compared to those from EMG signals and MN torques and from EMG signals and joint velocity, but only for the last primitive (Fig. 7A, Table 2).

Fig. 7 and Table 2 near here

Some of the observed differences between the directional tuning of muscle-torque, mechanical, and EMG primitives may be due to the different number of primitives obtained from noisier EMG signals. To address this issue, we have compared NMF weights from subjects with the number of EMG primitives matching the number of muscle-torque and mechanical primitives, to the NMF weights from the rest of the subjects with unmatched number of EMG primitives. The mean difference \pm confidence interval between the NMF weights from 3 subjects with three EMG primitives and 5 subjects with other numbers of EMG primitives $(2,4$, and 5$)$ was $0.005 \pm 0.025$, which was not significant $(p=0.65)$. This shows that the difference between muscle-torque, mechanical, and EMG primitives is not due to the larger noise in EMG signals.

\section{Discussion}

The hypothesis of our study was that primitives obtained from muscle contractions are more similar to kinetic primitives obtained from joint torques, than kinematic primitives obtained from joint angles and angular velocity signals. We found that when controlling for temporal evolution of primitives, the spatial distribution of EMG and MG primitives overlapped more than that of EMG and joint velocity primitives. However, no other statistical differences between the spatial distributions of kinetic and kinematic signals were observed. These results led us to reject the main hypothesis. Instead, our results suggest that motor primitives do not consist of signals of a single modality, but rather combine both kinetic and kinematic signals. 
The transformation from muscle activation to motion is non-linear and includes second order differential dynamics. Here, we have defined motor primitives as functional components of joint torques that are related to either gravity or interjoint coordination. Deriving primitives from dynamical signals like joint torques should result in a more linear relationship with neural control signals, if those primitives capture accurately the dynamics of neural signals. Indeed, we have found more shared variance between mechanical primitives and EMG primitives than between muscle-torque and EMG primitives. In particular, the primitive at the end of movement derived from the gravity torque component shared the most variance with the corresponding EMG primitive. In contrast, the primitive derived from joint velocity shared the least variance with the corresponding EMG primitive. This provides further supporting evidence for the dynamical, rather than kinematic, nature of neural motor commands (Caminiti et al., 1990; 1991; Scott, 1997; Scott and Kalaska, 1997). Neural commands may comprise both phasic and tonic components, similar to those identified in EMG during 3D pointing movements (d'Avella et al., 2008; Flanders, 1991). The required scaling of a hypothetical tonic command for different movement directions could reflect the corresponding changes in gravitational load on the arm, i.e. as predicted by the MG torque components in our study. Thus, the hypothetical tonic command compensating for gravity may constitute an anticipatory postural adjustment that accompanies movement (Massion, 1992). Alternatively, the hypothetical tonic command may be a spinal feedback response to changing gravitational load signaled by proprioceptors. Our results support the latter by indicating more robust differences in the shared variance at the end of movement (Fig. 7 and Table 2). The mechanism responsible for a feedback compensation for gravity may be akin to positive force feedback during locomotion based on afferent feedback from Golgi tendon organs to maintain load bearing (Pearson and Collins, 1993; Prochazka et al., 1997). Deriving motor primitives with decomposition methods is confounded by the indivisible interactions between muscle activations and the resulting movement (Santello et al., 2013; Tresch and Jarc, 2009). In other words, it is difficult to determine whether correlations between muscle activations cause correlations between motion-related signals, or it is the other way around. We too have observed the high degree of shared variance within all motion-related signals as evidenced by only three primitives present in both the kinematic and dynamic signals, consistent with results reported previously for reaching movements (Chiovetto et al., 2013). However, we have also shown that NMF weights, while being directionally tuned as reported previously (Chiovetto et al., 2010; 2013; d'Avella et al., 2006; TorresOviedo and Ting, 2007), were tuned very differently when obtained from kinematic, or kinetic, or EMG signals (Fig. 3). We observed a rather small subset of pairs of signals with more than $10 \%$ shared variance, many of which did not come from the same joint (Fig. 7). This suggests that the method of examining the overlap in directional tuning while controlling for common temporal dynamics may offer a way to identify the modality of control signals and to disambiguate it from inherent relationships across multiple types of motion-related signals.

The CNS is a hierarchal dynamical system with recurrent feedback loops (Prochazka and Yakovenko, 2007; Shenoy et al., 2013). The output of this system has been 
observed in electrophysiological studies in animals as sequential neural primitives that are recruited during a reaching motion and correspond to the different phases of movement (Yakovenko et al., 2011). Similarly, we have observed highly correlated activations of sequential EMG primitives (Fig. 4). These activations were shifted in time at physiological delays (Fig. 5), which are consistent with transcortical feedback loops (Lee et al., 1983). These results suggest that the timing of the activations of the NMF primitives may indicate the timing of feedback processing within the motor system. The predictable sequence of peaks in the activation of primitives may be a useful tool for diagnosing neural dysfunction (Olesh and Gritsenko, 2017).

Our results are also consistent with the idea that there are no "true" muscle primitives. This argument is illustrated by a recent study, which found that muscles are recruited flexibly without a consistent pattern of groupings across movements (Kutch et al., 2008). In our results these flexible muscle groupings may appear as highly variable NMF weights, which vary in directional tuning between movement planes and forward and backward directions. Such flexible recruitment may be enabled by the dynamical nature of the nervous system, which could fully imbed the complex limb dynamics and its interaction with the external world in dedicated neural modules called internal models (Gomi and Kawato, 1997; Lackner and Dizio, 1994; Sabes, 2000; Shadmehr and Mussa Ivaldi, 1994; Wolpert and Kawato, 1998). These internal models or embodied neural dynamical systems would calculate the required muscle activation patterns specifically for a given class of similar movements, which may represent a learned task or a unit of motor memory (Haruno et al., 2001; Wolpert and Kawato, 1998).

\subsection{Conclusions}

In conclusion, our results have shown that when controlling for the temporal evolution of primitives, the primitives obtained from muscle activity and gravitational torques shared the most variance. This suggests that motor primitives do not consist of signals of a single modality, but rather combine both kinetic and kinematic signals.

\section{Conflict of Interest Statement}

The authors declare no conflicts of interest.

\section{Authors and Contributors}

EO contributed to the design of the study, subject recruitment, data collection, data analysis, and writing of the manuscript. BP contributed to the data collection and analysis, and to writing of the manuscript. VG contributed to the design of the study, analysis of data, and writing of the manuscript. 


\section{Funding}

This research was sponsored by NIH/NIGMS U54GM104942 (EO) providing student fellowship, NIH P20GM109098 providing salary support (VG, BP) and supplies, NIH P30GM103503 providing equipment support. The content is solely the responsibility of the authors and does not necessarily represent the official views of the NIH.

\section{Acknowledgments}

The authors wish to thank Dr. Sergiy Yakovenko for his contribution to the discussion of analysis in this study and Dr. Robert L. Goodman and Dr. Amy J. Bastian for their critical review of this manuscript.

\section{References}

Bastian, A. J., Martin, T. A., Keating, J. G., and Thach, W. T. (1996). Cerebellar ataxia: abnormal control of interaction torques across multiple joints. J Neurophysiol 76, 492-509.

Berniker, M., Jarc, A., Bizzi, E., and Tresch, M. C. (2009). Simplified and effective motor control based on muscle synergies to exploit musculoskeletal dynamics. PNAS 106, 7601-7606. doi:10.1073/pnas.0901512106.

Bizzi, E., Mussa Ivaldi, F., and Giszter, S. (1991). Computations underlying the execution of movement: a biological perspective. Science 253, 287-291.

Caminiti, R., Johnson, P. B., and Urbano, A. (1990). Making arm movements within different parts of space: dynamic aspects in the primate motor cortex. $J$ Neurosci 10, 2039-2058.

Caminiti, R., Johnson, P., Galli, C., Ferraina, S., and Burnod, Y. (1991). Making arm movements within different parts of space: the premotor and motor cortical representation of a coordinate system for reaching to visual targets. J Neurosci 11, 1182-1197.

Chiovetto, E., Berret, B., and Pozzo, T. (2010). Tri-dimensional and triphasic muscle organization of whole-body pointing movements. Neurosci 170, 1223-1238. doi:10.1016/j.neuroscience.2010.07.006.

Chiovetto, E., Berret, B., Delis, I., Panzeri, S., and Pozzo, T. (2013). Investigating reduction of dimensionality during single-joint elbow movements: a case study on muscle synergies. Front Comput Neurosci 7, 1:12.

doi:10.3389/fncom.2013.00011.

Chvatal, S. A., Torres-Oviedo, G., Safavynia, S. A., and Ting, L. H. (2011). Common muscle synergies for control of center of mass and force in nonstepping and 
stepping postural behaviors. J Neurophysiol 106, 999-1015.

doi:10.1152/jn.00549.2010.

d'Avella, A., and Lacquaniti, F. (2013). Control of reaching movements by muscle synergy combinations. Front Comput Neurosci 7, 42.

doi:10.3389/fncom.2013.00042.

d'Avella, A., Fernandez, L., Portone, A., and Lacquaniti, F. (2008). Modulation of phasic and tonic muscle synergies with reaching direction and speed. $J$ Neurophysiol 100, 1433-1454. doi:10.1152/jn.01377.2007.

d'Avella, A., Portone, A., Fernandez, L., and Lacquaniti, F. (2006). Control of fastreaching movements by muscle synergy combinations. Journal of Neuroscience 26, 7791-7810. doi:10.1523/JNEUROSCI.0830-06.2006.

Debicki, D. B., and Gribble, P. L. (2005). Persistence of inter-joint coupling during single-joint elbow flexions after shoulder fixation. Experimental brain research 163, 252-257.

Dounskaia, N. V., and Wang, W. (2014). A preferred pattern of joint coordination during arm movements with redundant degrees of freedom.J Neurophysiol 112, 1040-1053. doi:10.1152/jn.00082.2014.

Fisk, J., Lackner, J. R., and Dizio, P. (1993). Gravitoinertial force level influences arm movement control. J Neurophysiol 69, 504-511.

Flanders, M. (1991). Temporal patterns of muscle activation for arm movements in three-dimensional space. J Neurosci 11, 2680-2693.

Gentili, R., Cahouet, V., and Papaxanthis, C. (2007). Motor planning of arm movements is direction-dependent in the gravity field. Neurosci 145, 20-32. doi:10.1016/j.neuroscience.2006.11.035.

Giszter, S. F., Mussa-Ivaldi, F. A., and Bizzi, E. (1993). Convergent force fields organized in the frog's spinal cord. J Neurosci 13, 467-491.

Gomi, H., and Kawato, M. (1997). Human arm stiffness and equilibrium-point trajectory during multi-joint movement. Biol Cybern 76, 163-171.

Gribble, P. L., and Ostry, D. J. (1999). Compensation for Interaction Torques During Single- and Multijoint Limb Movement. J Neurophysiol 82, 2310-2326.

Gritsenko, V., Kalaska, J. F., and Cisek, P. (2011). Descending corticospinal control of intersegmental dynamics. J Neurosci 31, 11968-11979.

doi:10.1523/JNEUROSCI.0132-11.2011. 
Gritsenko, V., Yakovenko, S., and Kalaska, J. F. (2009). Integration of predictive feedforward and sensory feedback signals for online control of visually guided movement. J Neurophysiol 102, 914-930. doi:10.1152/jn.91324.2008.

Haruno, M., Wolpert, D. M., and Kawato, M. (2001). Mosaic model for sensorimotor learning and control. Neural Comput 13, 2201-2220. doi:10.1162/089976601750541778.

Hollerbach, J. M., and Flash, T. (1982). Dynamic interactions between limb segments during planar arm movement. Biol Cybern 44, 67-77.

Kutch, J. J., and Valero-Cuevas, F. J. (2012). Challenges and new approaches to proving the existence of muscle synergies of neural origin. PLoS Comput. Biol. 8, e1002434. doi:10.1371/journal.pcbi.1002434.

Kutch, J. J., Kuo, A. D., Bloch, A. M., and Rymer, W. Z. (2008). Endpoint force fluctuations reveal flexible rather than synergistic patterns of muscle cooperation. J Neurophysiol 100, 2455-2471. doi:10.1152/jn.90274.2008.

Lackner, J. R., and Dizio, P. (1994). Rapid adaptation to Coriolis force perturbations of arm trajectory.J Neurophysiol 72, 299-313.

Le Seac'h, A. B., and McIntyre, J. (2007). Multimodal reference frame for the planning of vertical arms movements. Neurosci Lett 423, 211-215. doi:10.1016/j.neulet.2007.07.034.

Lee, R. G., Murphy, J. T., and Tatton, W. G. (1983). Long-latency myotatic reflexes in man: mechanisms, functional significance, and changes in patients with Parkinson's disease or hemiplegia. Adv Neurol 39, 489-508.

Massion, J. (1992). Movement, posture and equilibrium: interaction and coordination. Prog Neurobiol 38, 35-56.

Mussa Ivaldi, F. (1999). Modular features of motor control and learning. Curr Opin Neurobiol 9, 713-717.

Mussa Ivaldi, F., and Bizzi, E. (2000). Motor learning through the combination of primitives. Philos Trans R Soc Lond B 355, 1755-1769.

Olesh, E. V., and Gritsenko, V. (2017). Linking post-stroke movement impairment to mechanistic changes in the neural control of movement. In: Combined Sections Meeting; 2017 Feb 15-18; San Antonio, TX. p. 1280.

Papaxanthis, C., Pozzo, T., and McIntyre, J. (2005). Kinematic and dynamic processes for the control of pointing movements in humans revealed by short-term exposure to microgravity. Neurosci 135, 371-383. doi:10.1016/j.neuroscience.2005.06.063. 
Papaxanthis, C., Pozzo, T., Popov, K. E., and McIntyre, J. (1998). Hand trajectories of vertical arm movements in one-G and zero-G environments. Evidence for a central representation of gravitational force. Experimental brain research 120, 496-502.

Pearson, K. G., and Collins, D. F. (1993). Reversal of the influence of group Ib afferents from plantaris on activity in medial gastrocnemius muscle during locomotor activity. J Neurophysiol 70, 1009-1017.

Pigeon, P., Bortolami, S., Dizio, P., and Lackner, J. R. (2003). Coordinated turn-andreach movements. I. Anticipatory compensation for self-generated Coriolis and interaction torques. J Neurophysiol 89, 276-289.

doi:10.1152/jn.00159.2001.

Pozzo, T., Papaxanthis, C., Stapley, P., and Berthoz, A. (1998). The sensorimotor and cognitive integration of gravity. Brain Res Brain Res Rev 28, 92-101.

Prochazka, A., and Yakovenko, S. (2007). The neuromechanical tuning hypothesis. Prog Brain Res 165, 255-265. doi:10.1016/S0079-6123(06)65016-4.

Prochazka, A., Gillard, D. M., and Bennett, D. J. (1997). Positive force feedback control of muscles. J Neurophysiol 77, 3226-3236.

Sabes, P. N. (2000). The planning and control of reaching movements. Curr Opin Neurobiol 10, 740-746.

Sainburg, R. L., Ghez, C., and Kalakanis, D. (1999). Intersegmental Dynamics Are Controlled by Sequential Anticipatory, Error Correction, and Postural Mechanisms. J Neurophysiol 81, 1045-1056.

Sainburg, R. L., Ghilardi, M., Poizner, H., and Ghez, C. (1995). Control of limb dynamics in normal subjects and patients without proprioception. $J$ Neurophysiol 73, 820-835.

Santello, M., Baud-Bovy, G., and Jörntell, H. (2013). Neural bases of hand synergies. Front Comput Neurosci 7, 23. doi:10.3389/fncom.2013.00023.

Scott, S. H. (1997). Comparison of onset time and magnitude of activity for proximal arm muscles and motor cortical cells before reaching movements. $J$ Neurophysiol 77, 1016-1022.

Scott, S. H., and Kalaska, J. F. (1997). Reaching movements with similar hand paths but different arm orientations. I. Activity of individual cells in motor cortex. $J$ Neurophysiol 77, 826-852. 
Shabbott, B. A., and Sainburg, R. L. (2008). Differentiating between two models of motor lateralization. J Neurophysiol 100, 565-575.

doi:10.1152/jn.90349.2008.

Shadmehr, R., and Mussa Ivaldi, F. (1994). Adaptive representation of dynamics during learning of a motor task. J Neurosci 14, 3208-3224.

Shenoy, K. V., Sahani, M., and Churchland, M. M. (2013). Cortical control of arm movements: a dynamical systems perspective. Annu Rev Neurosci 36, 337359. doi:10.1146/annurev-neuro-062111-150509.

Tagliabue, M., Ciancio, A. L., Brochier, T., Eskiizmirliler, S., and Maier, M. A. (2015). Differences between kinematic synergies and muscle synergies during twodigit grasping. Front Hum Neurosci 9, 1-17. doi:10.3389/fnhum.2015.00165.

Talkington, W. J., Pollard, B. S., Olesh, E. V., and Gritsenko, V. (2015). Multifunctional Setup for Studying Human Motor Control Using Transcranial Magnetic Stimulation, Electromyography, Motion Capture, and Virtual Reality. J Vis Exp, e52906-e52906. doi:10.3791/52906.

Ting, L. H. (2007). Dimensional reduction in sensorimotor systems: a framework for understanding muscle coordination of posture. Prog Brain Res 165, 299-321. doi:10.1016/S0079-6123(06)65019-X.

Torres-Oviedo, G., and Ting, L. H. (2007). Muscle synergies characterizing human postural responses. J Neurophysiol 98, 2144-2156. doi:10.1152/jn.01360.2006.

Torres-Oviedo, G., Macpherson, J. M., and Ting, L. H. (2006). Muscle synergy organization is robust across a variety of postural perturbations. $J$ Neurophysiol 96, 1530-1546. doi:10.1152/jn.00810.2005.

Tresch, M. C., and Jarc, A. (2009). The case for and against muscle synergies. Curr Opin Neurobiol 19, 601-607. doi:10.1016/j.conb.2009.09.002.

Tresch, M. C., Cheung, V. C. K., and d'Avella, A. (2006). Matrix factorization algorithms for the identification of muscle synergies: evaluation on simulated and experimental data sets. J Neurophysiol 95, 2199-2212. doi:10.1152/jn.00222.2005.

Tresch, M. C., Saltiel, P., and Bizzi, E. (1999). The construction of movement by the spinal cord. Nat Neurosci 2, 162-167. doi:10.1038/5721.

Valero-Cuevas, F. J., Cohn, B. A., Yngvason, H. F., and Lawrence, E. L. (2015). Exploring the high-dimensional structure of muscle redundancy via subjectspecific and generic musculoskeletal models. J Biomech 48, 2887-2896. doi:10.1016/j.jbiomech.2015.04.026. 
Winter, D. A. (2009). Biomechanics and Motor Control of Human Movement. 4 ed. Hoboken, New Jersey: John Wiley \& Sons.

Wolpert, D. M., and Kawato, M. (1998). Multiple paired forward and inverse models for motor control. Neural Netw 11, 1317-1329.

Yakovenko, S., Krouchev, N. I., and Drew, T. (2011). Sequential activation of motor cortical neurons contributes to intralimb coordination during reaching in the cat by modulating muscle synergies. J Neurophysiol 105, 388-409. doi:10.1152/jn.00469.2010.

\section{Figure and Table Legends}

Figure 1. Experimental setup and analysis flow. (A) Illustration showing the locations of reaching targets, arranged in a semi-spherical pattern in VR, relative to the physical location of the subject. The central target is shown in red and one of the goal targets is shown in green. (B) Schematic representation of analysis flow. IDM stands for inverse dynamic model. NMF1, NMF2, and MNF3 indicate separate analyses applied to EMG, muscle torque, and other kinetic and kinematic data respectively. Mathematical symbols match those used in formulae in Methods.

Figure 2. Examples of NMF analysis of EMG. (A) Average muscle activation (solid lines) from two muscles during one movement for one subject (plotted with standard deviation from 15 repetitions of the same movement). The subject shown here required 4 primitives for EMG VAF > 95\%. The activation profiles from these primitives were categorized as A, B, D and E based on the order of their peaks (see Methods). (B) Illustration of weights across movements for the two EMG signals. The NMF weights for each primitive and each movement direction is the distance in the polar coordinates, the angle is based on the direction of motion in one plane, or the reaching target location relative to the starting target.

\section{Figure 3. Example motion signals, muscle activity, and corresponding NMF} weights for a single subject. The central polar plots show normalized weights for the first NMF primitive plotted as in Fig. 2B. The temporal profiles of signals from which the NMF weights were obtained are shown around the polar plots in matching colors. All signals are normalized in time and amplitude as described in Methods for NMF analysis. (A) Joint angles in time and corresponding weights from NMF3. Averages (solid lines) and standard deviations (shaded areas) are across movement repetitions. (B) Angular velocity in time and corresponding weights from NMF3, formatted as in (A). (C) Muscle torques in time and corresponding weights from NMF2, formatted as in (A). (D) MG torque in time and corresponding weights from NMF3, formatted as in (A). (E) MN torque in time and corresponding weights from NMF3, formatted as in (A). (F) EMG signals in time and corresponding weights from NMF1. Averages (solid lines) and standard errors of the mean (shaded areas) are across movement repetitions. Muscle abbreviations are as described in Methods. 
Figure 4. Temporal activation profiles of EMG, muscle-torque, and mechanical primitives. Average normalized activation profiles (solid lines) and standard deviations (shaded area) across all eight subjects are plotted for each activation. The activation profiles were arranged based on the occurrence of the first peak and labeled A through $\mathrm{C}$ for muscle-torque (blue) and mechanical (black) primitives. EMG activation profiles were labeled A through C (red). Titles show the labels for activation profiles from each type of primitive that are plotted on the same plot. Both temporal and amplitude values for all activation profiles were normalized as described in Methods.

Figure 5. Cross-correlation lag times and peaks of NMF activation profiles. (A) Lag times that correspond to maximal correlations between activation profiles of EMG primitives. Red lines indicate the median value across subjects with the 25 th and 75th percentiles marked by the edges of each box. (B) Peak times of each activation profile of EMG primitive per subject. (C) Lag times that correspond to maximal correlations between activation profiles of EMG and muscle-torque primitives. Plot is formatted as in A. (D) Peak times of activation profiles of EMG primitives and the corresponding muscle-torque primitives per subject. (E) Lag times that correspond to maximal correlations between activation profiles of EMG primitives and mechanical primitives. Plot is formatted as in A. (F) Peak times of activation profiles of EMG primitives and the corresponding mechanical primitives per subject.

Figure 6. Shared variance between weights from NMF1 on EMG and NMF2 on muscle torques. The colors of circles indicate $r 2$ values between weights for corresponding signals across all movements averaged across subjects. Muscle abbreviations are as described in Methods. F/E stands for flexion/extension; Ab/Ad stands for abduction/adduction; Pro/Sup stands for pronation/supination.

Figure 7. Shared variance between weights from NMF1 on EMG and NMF3 on kinematic and kinetic signals. The colors of circles indicate $r 2$ values between weights for corresponding signals across all movements averaged across subjects. Muscle abbreviations are as described in Methods. F/E stands for flexion/extension; $\mathrm{Ab} /$ Ad stands for abduction/adduction; Pro/Sup stands for pronation/supination.

\section{Tables}

Table 1. rANOVA1 on shared variance between weights of NMF 1 on EMG and weights of NMF2 on muscle torques.

\begin{tabular}{|l|l|l|l|}
\hline rANOVA & Degrees of Freedom & $\boldsymbol{F}$ & $\boldsymbol{p}$ \\
\hline Factors & 119 & 3.58 & $\mathbf{0 . 0 1}$ \\
\hline Gender x Factors & 119 & 0.84 & 0.53 \\
\hline
\end{tabular}




\begin{tabular}{|l|l|l|l|}
\hline rANOVA & Degrees of Freedom & $\boldsymbol{F}$ & $\boldsymbol{p}$ \\
\hline Multiple comparisons & & Difference & $\boldsymbol{p}$ \\
\hline Factor Primitive & A - C/E & -0.02 & 0.09 \\
\hline Factor Joint & across - within & 0.002 & 0.65 \\
\hline
\end{tabular}

Table 2. rANOVA2 on shared variance between weights of NMF 1 on EMG and weights of NMF3 on kinematic and kinetic signals.

\begin{tabular}{|l|l|l|l|}
\hline rANOVA & Degrees of Freedom & $\boldsymbol{F}$ & $\boldsymbol{p}$ \\
\hline Factors & 479 & 3.32 & $\mathbf{0 . 0 2}$ \\
\hline Gender x Factors & 479 & 0.88 & 0.51 \\
\hline Multiple comparisons & & Difference & $\boldsymbol{p}$ \\
\hline Primitive A & velocity - angle & -0.023 & 0.15 \\
\hline Primitive A & velocity - MG torque & -0.003 & 0.93 \\
\hline Primitive A & velocity - MN torque & 0.013 & 0.64 \\
\hline Primitive A & angle - MG torque & 0.019 & 0.07 \\
\hline Primitive A & angle - MN torque & 0.036 & 0.18 \\
\hline Primitive A & MG torque - MN torque & 0.017 & 0.45 \\
\hline Primitive C/E & velocity - angle & -0.012 & 0.47 \\
\hline Primitive C/E & velocity - MG torque & -0.033 & $\mathbf{0 . 0 1}$ \\
\hline Primitive C/E & velocity - MN torque & 0.024 & 0.1 \\
\hline Primitive C/E & angle - MG torque & -0.021 & 0.16 \\
\hline Primitive C/E & angle - MN torque & 0.036 & 0.05 \\
\hline Primitive C/E & MG torque - MN torque & 0.056 & $\mathbf{0 . 0 2}$ \\
\hline
\end{tabular}


Figure 1
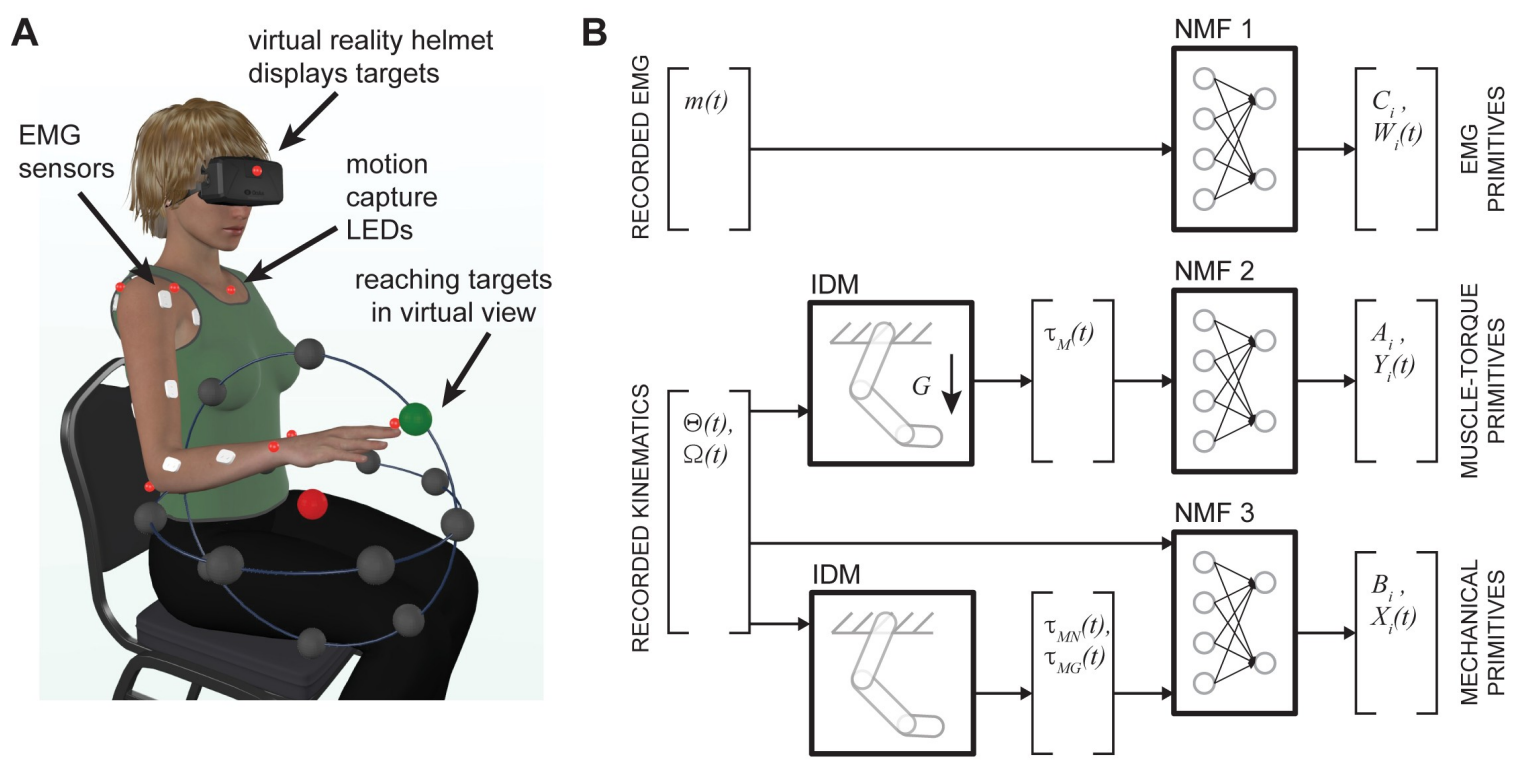
Figure 2

A
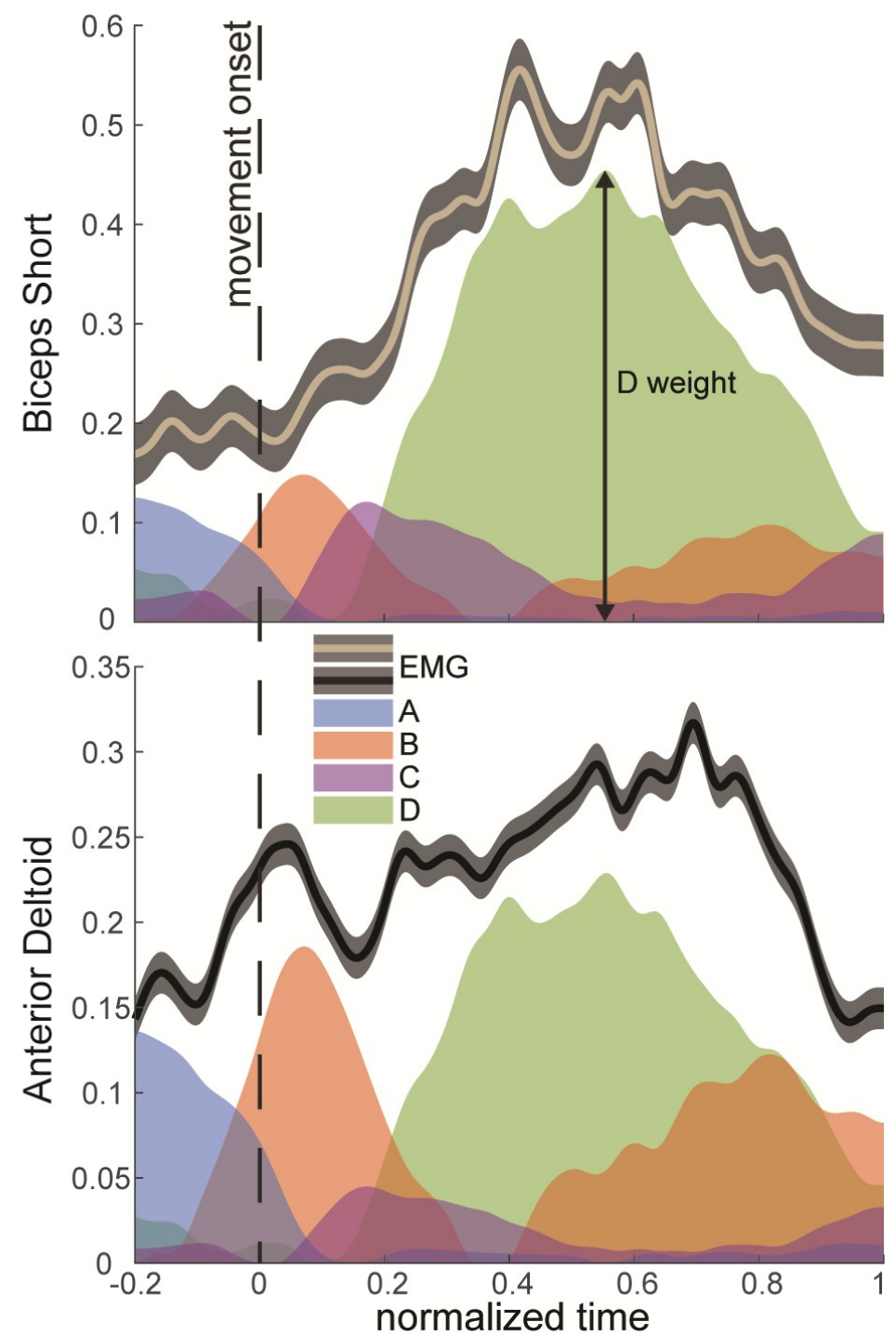

B

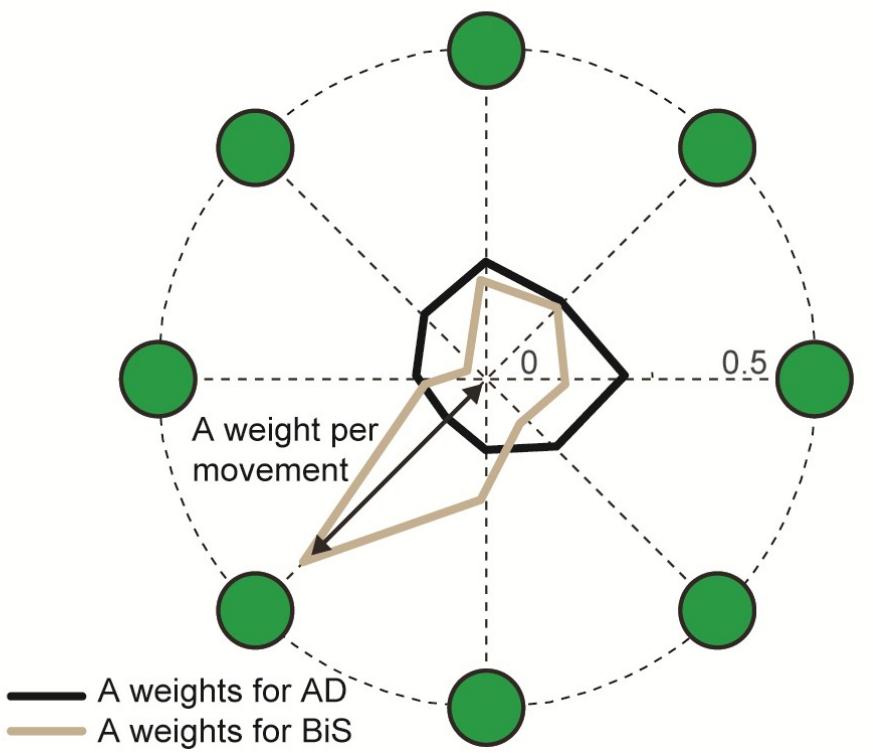


Figure 3

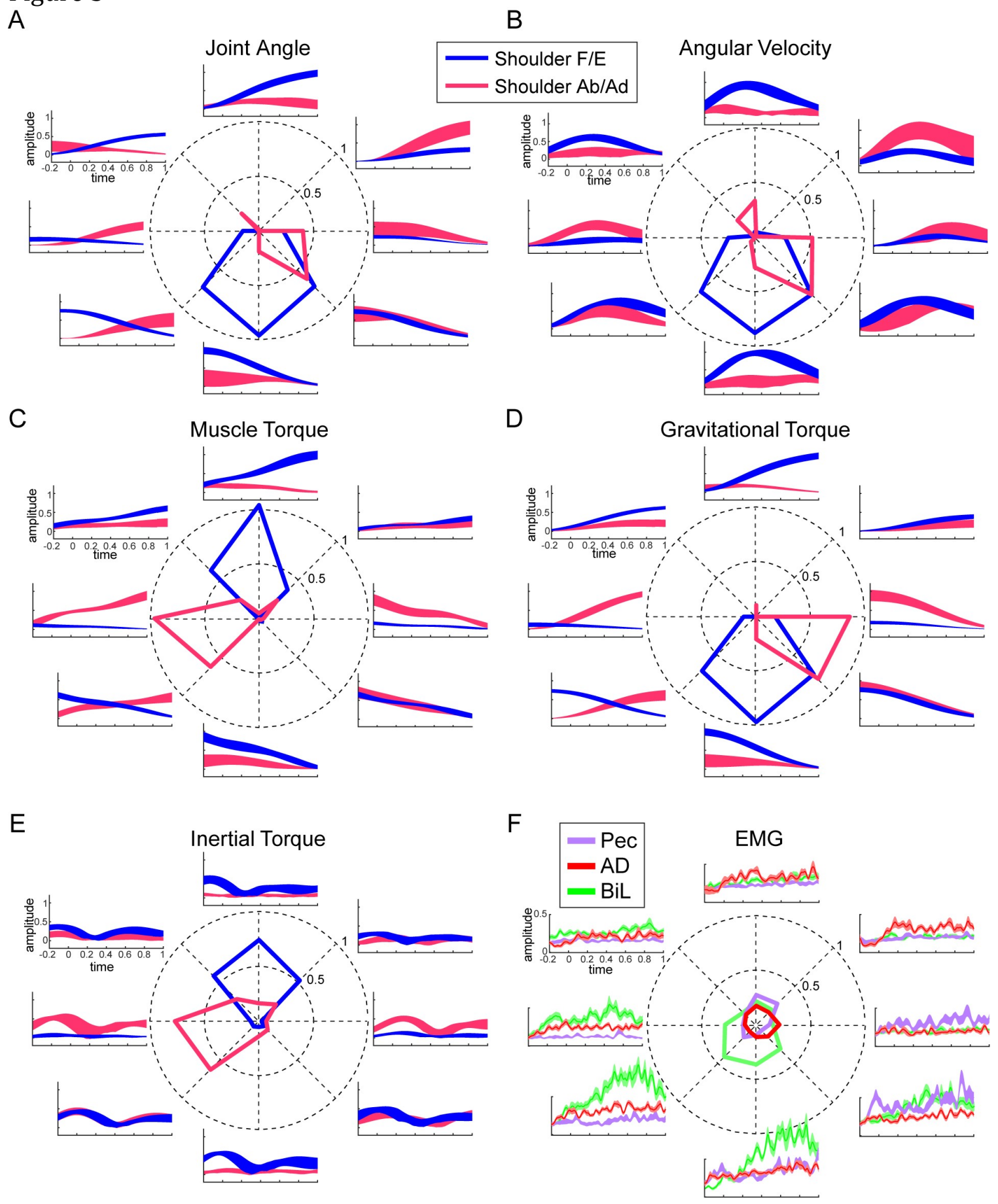


Figure 4
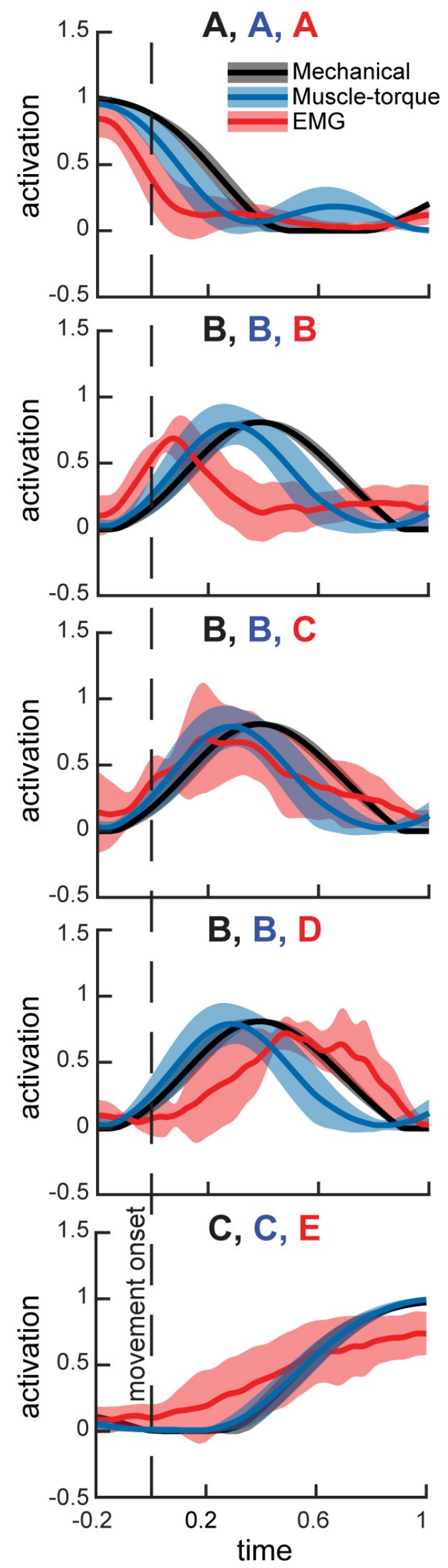
Figure 5
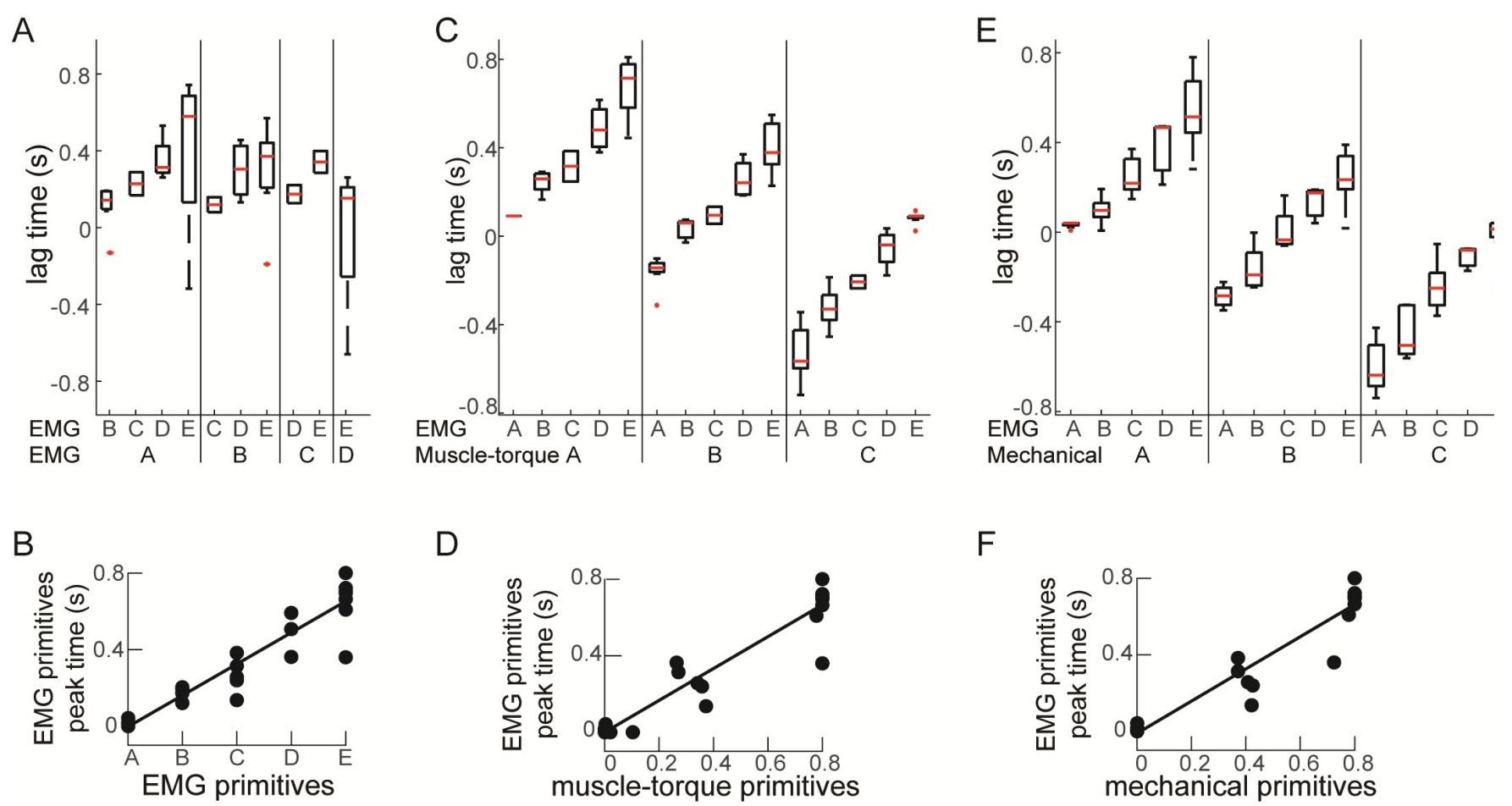

D

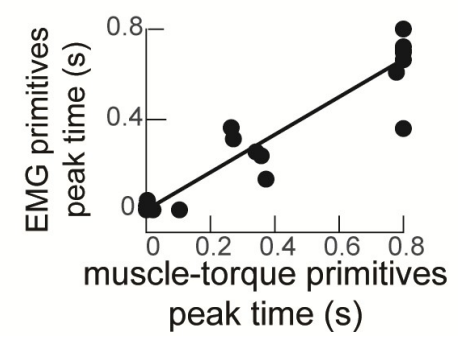

F

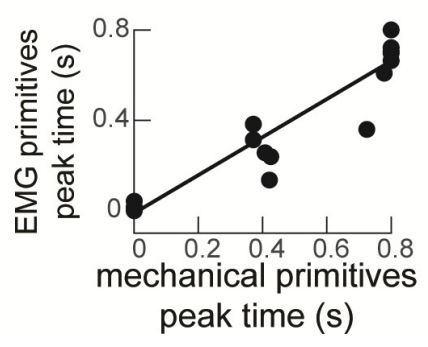


Figure 6
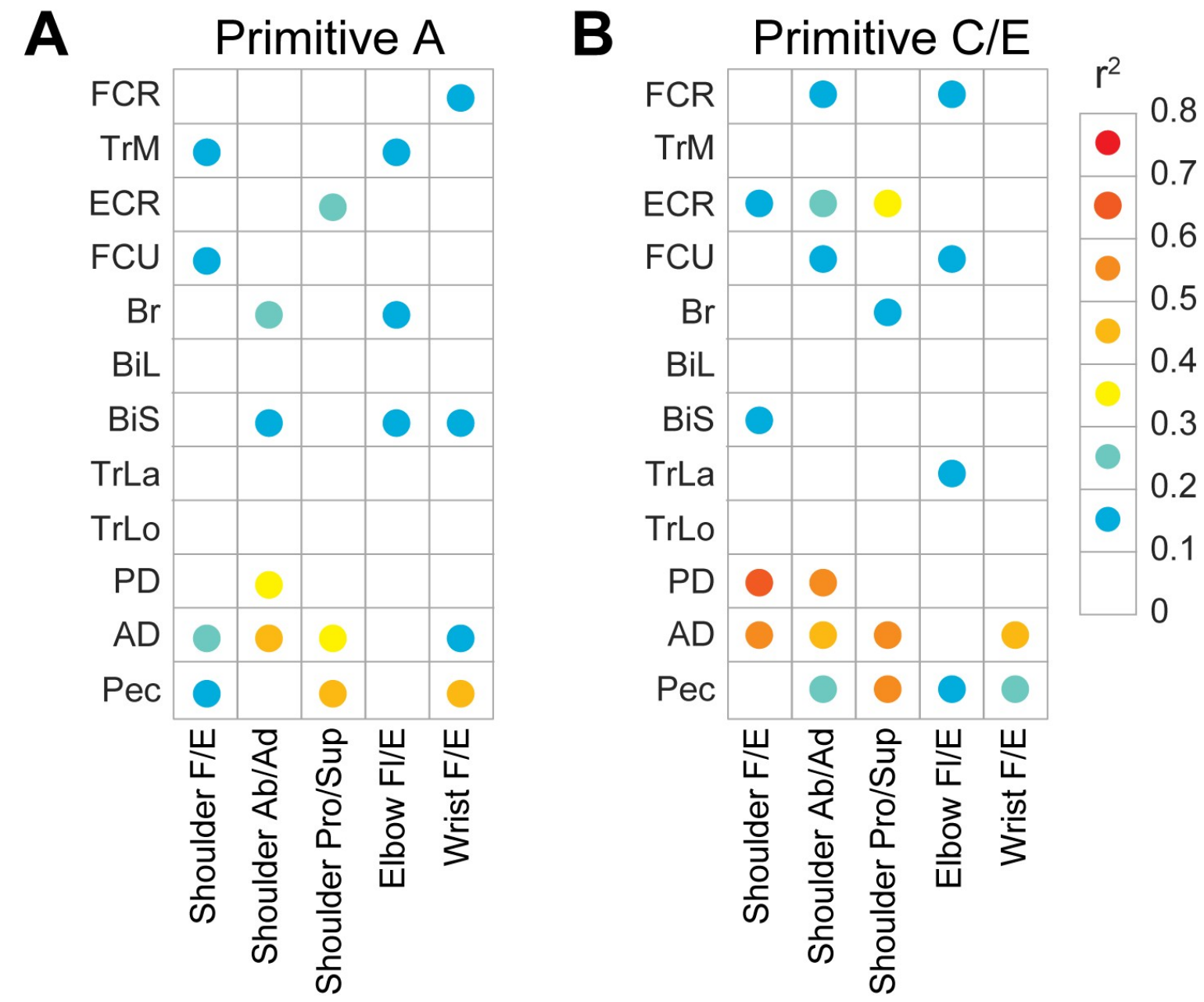
Figure 7

A

Primitive A

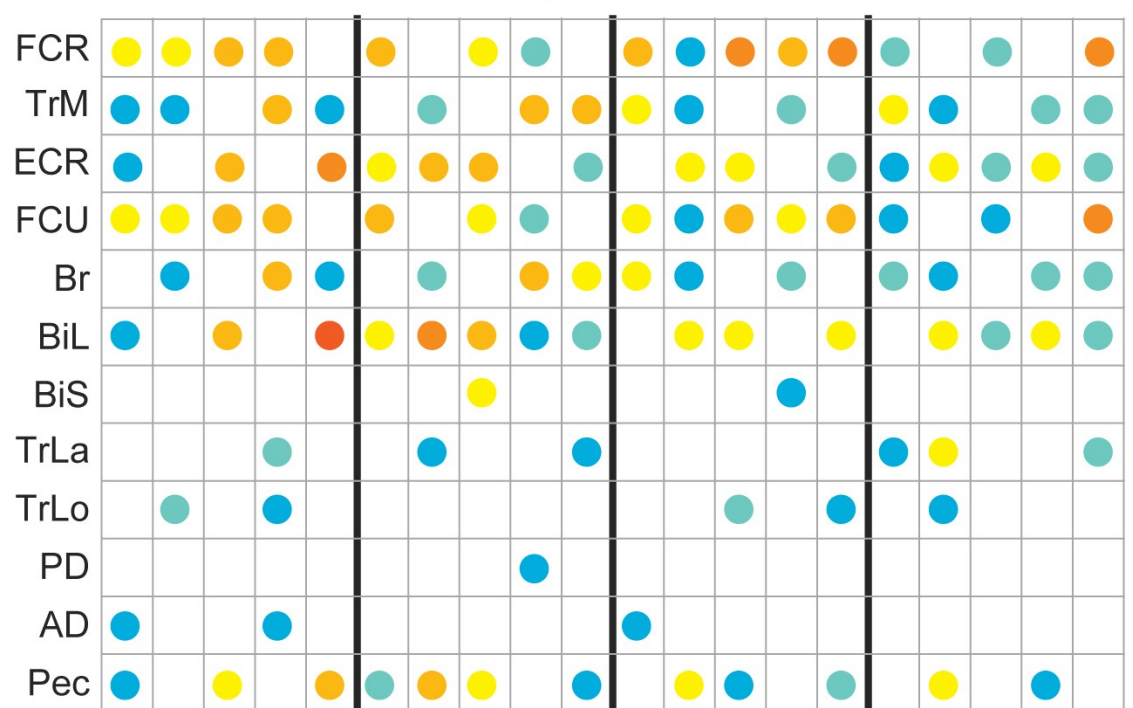

山웅의

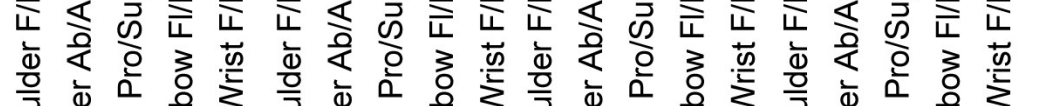

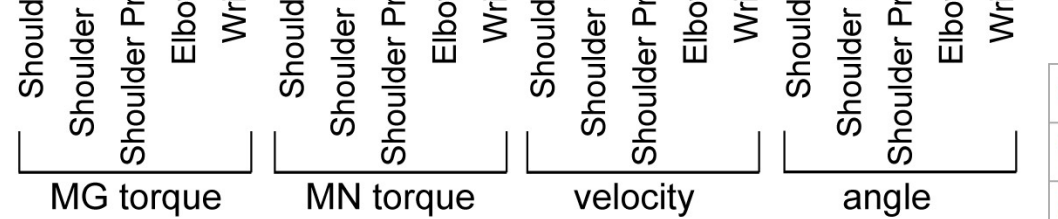

B

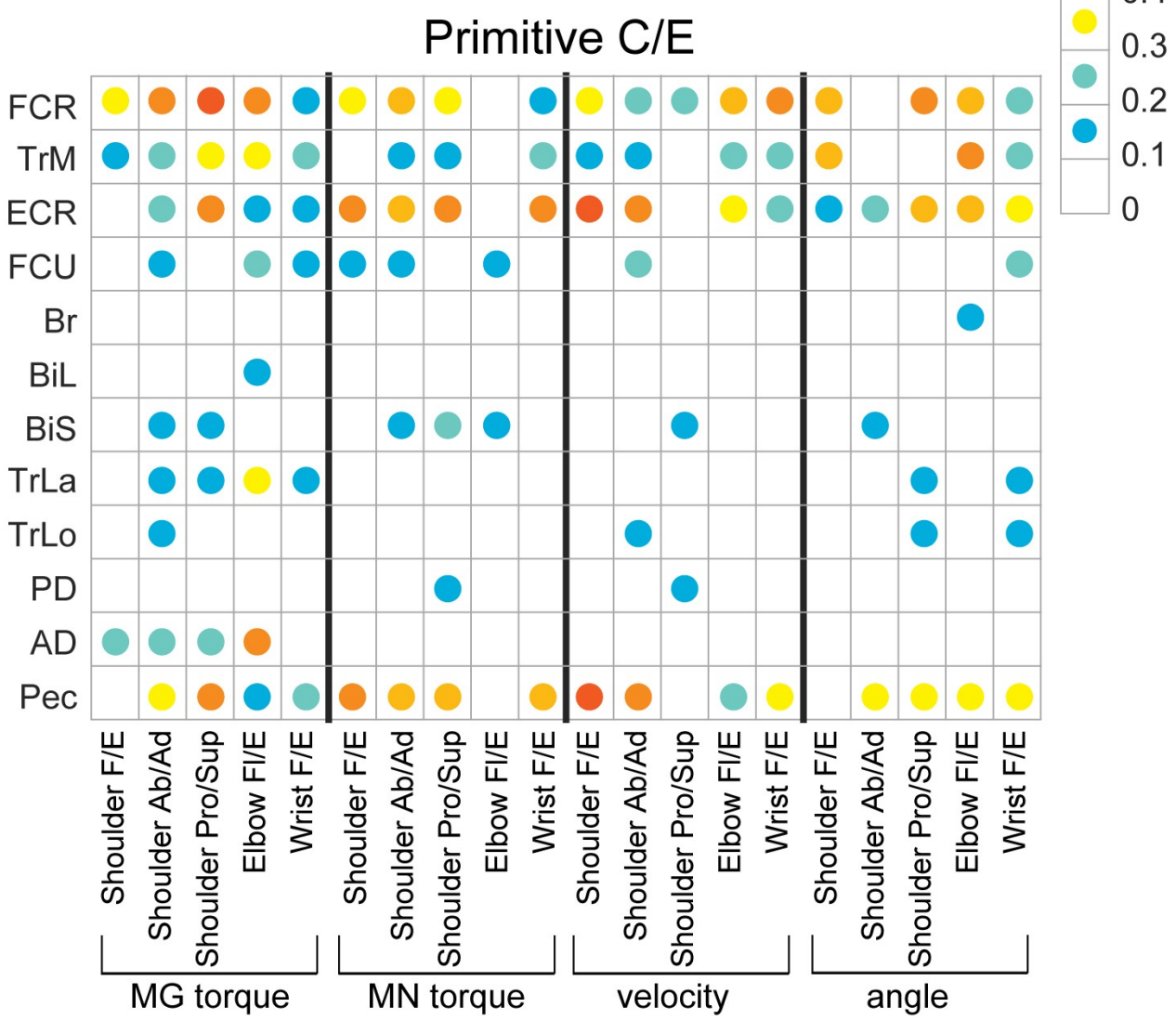




\section{Chapter 5}

Linking post-stroke movement impairment to mechanistic changes in the neural control of movement

\section{Authors and Contributors}

Erienne Olesh contributed to the design of the study, subject recruitment, data collection, data analysis, and writing of the manuscript. Brad Pollard contributed to the data collection. Valeriya Gritsenko contributed to the design of the study, analysis of data, and writing of the manuscript. 


\section{Introduction}

The effect a stroke can have on an individual is widely variable, ranging from verbal and visual deficits, to cognitive and motor impairment. Perhaps the most noted, and often-studied impairment resulting from a stroke is movement dysfunction (Wade, 1992). Eighty percent of stroke survivors are diagnosed with motor impairment after a stroke and of those, over fifty percent will retain some form of long-term motor impairment (Langhorne et al., 2009; Wahl and Schwab, 2014).

Motor impairment, in the context of a stroke, is a consequence of damage caused by infarct to the motor or premotor cortex, motor tracts, or pathways associated with movement production or regulation. The ensuing impairment can be defined as the loss or limitation of muscle control, movement, or mobility and most often affects the face, arm, leg, or any combination of these, on the contralateral side of the body from the stroke (Warlow et al, 2008). These deficits can greatly decrease an individual's ability to care for their self, leading to the need for long term assistance. Even for individuals who require little assistance after a stroke, the disruption of normal movement can be frustrating and limit their involvement in social activities they previously enjoyed.

Numerous rehabilitation methods have been proposed and studied for their efficacy in ameliorating post stroke motor deficits. However, a great number of these efforts have focused on the training of compensatory strategies. Although this strategy allows a patient to potentially regain some independence, it does not aim to improve the underlying muscle impairment. This can have adverse effects and limit the long-term rehabilitation potential of those individuals by creating aberrant patterns of muscle activity (Takeuchi and Izumi, 2012; Lough et al., 1984). In an attempt to improve rehabilitation strategies and gain a better understanding of the changes that occur to the control of movement in stroke patients, neuroscience and motor control research has expanded to investigate the underlying causes that may lead to chronic motor impairment.

Recent studies of stroke patients have suggested that long-term motor impairment may result from altered motor primitive composition (Cheung et al., 2012; Roh et al., 2015; Clark et al., 2010; Cruz and Dhaher, 2008). Motor primitives, sometimes also referred to as muscle synergies, can be described as a group of muscles or movement parameter with shared variance that co-activate in a manner capable of producing coordinated movement. These primitives have been suggested as a method by which the central nervous system reduces the complexity of the musculoskeletal system (Bizzi et al., 1991; Tresch et al., 2002; Ting, 2007). Results from Cheung and colleagues describe two general patterns of primitive reorganization they observed in motor impaired stroke patients. These patterns included 1.) fractionation, where one primitive is split into several new primitives, and 2.) merging, where several primitives blend into one large, less specific primitive (Cheung et al., 2012). It has also been demonstrated that intensive rehabilitation programs can alter the structure of motor primitives in stroke patients and that these changes correlate with improved performance on motor tasks (Tropea et al., 2013). 
Although potentially insightful, studying a stroke patient's recovery in regards to motor primitives has pitfalls. The theory of motor primitives has become less favorable as a mechanism of linking motion to muscle activity due to the inherent limitations of the methods used in identifying primitives. Previously, our lab has tested non-negative matrix factorization (NMF), one of the most common methods used to extract motor primitives to determine its usefulness in comparing muscle activity to resulting motion (see chapter 4 for more details). In this experiment, primitives extracted from muscle, motion and dynamic data were compared, and no consistent overlap in the directional tuning between these signals was found. These results suggest that although decomposition methods, such as $\mathrm{NMF}$, may group signals with similar shared variance from one data type (EMG for instance) into primitives, that this relationship is not necessarily maintained across other biomechanical signals. It is therefor problematic to infer levels of movement impairment based on the composition of motor primitives, as this may not be truly reflective of an individual's capability.

Other research into the changes that arise in the neural control of movement post stroke have suggested that movement dysfunction is a result of anatomical changes. Alterations to descending motor pathways and increased ipsilateral projections have been suggested to increase improper muscle co-contractions and joint stiffness leading to improper movement production (Levin, 1996; Gowland et al., 1992; Trombly, 1992). Yet other researchers have suggested that the anatomical changes may be more linked to an increase in devoted cortex space to the less impaired limb. Work by Kim and colleagues demonstrated that post stroke rats that were trained to have movement preference with their less impaired limb had an increase in cortical area devoted to that limb (Kim et al., 2015). Although these results are not necessarily surprising, this study highlights the importance of stroke patients becoming reliant on their less impaired limb and the negative consequences this can have on the individual's long-term rehabilitation outcome.

It remains critical to the progression towards better rehabilitation programs that the link between underlying muscle deficits and the resulting movement is understood (Lough et al., 1984). It is particularly important to improve our understanding of the altered patterns of muscle activity that arise in response to movements with varying levels of forces and torques. As our limbs move through space, they encounter different forces and torques, which create specific needs that must be compensated for by changes in muscle activity. The lack of ability to compensate for these forces may play a role in the long-term motor impairment we see in a significant number of stroke survivors. It was therefor the aim of this project to investigate the relationship between muscle activity of motor impaired stroke patients and the dynamic needs of different movement types. It is our hope that this work will allow for better-informed rehabilitation services that focus on retraining the underlying muscular changes that lead to post stroke motor impairment. 


\section{Methods \\ Experimental Design}

To better understand the connection between muscle activity and the resulting motion in stroke patients, we recruited eight chronically motor impaired stroke survivors to perform fourteen center out reaching task. Patients were included if they had suffered from one unilateral stroke and had no other confounding injuries or diseases that would impact movement performance. Stroke location was confirmed by either magnetic resonance imagining or computerized tomography as read and assessed by a physician. Patients were excluded if they could not produce visible movement with their shoulder and elbow, or if they were unable to provide written consent to participate. Age match control subjects were also recruited from West Virginia University and the surrounding area. Control subjects were excluded if they had any significant health conditions, had ever suffered from a stroke, or had any sever injuries to their upper extremities that would affect their movement capability.

Figure 1: Experimental Set up Electromyography was recorded from twelve muscles of the arm and shoulder. Motion capture data was recorded using the PhaseSpace motion capture system. Light emitting diodes were placed on anatomical landmarks of the arm, shoulder, and trunk. The Oculus Rift virtual reality head set was used to project targets to the patient in a randomized order. Targets were arranged in a semi-spherical pattern around a central starting target. Targets were illuminated in green to indicate movement to that target.

Reaching tasks were directed through the use of a virtual reality system that randomly displayed a single target at a time (Oculus Rift head set and Vizard virtual environment). Targets were arranged in a semi-spherical pattern around a central starting location that was adjusted to each individual such that the initial posture of the arm was in the following confirmation: zero degrees shoulder flexion, ninety degrees elbow flexion, zero degrees flexion of the wrist (Fig. 1). The distance between the central target 
and all exterior targets was scaled to be equal to thirty percent of each patients total arm length as measured from the anterior acromial point of the shoulder to the distal end of the index finger.

Patients were instructed to reach to the peripheral target when the target appeared green and to return to the central target once the peripheral target turned red, indicating a successful movement. Patients were instructed to move as quickly but as accurately as possible. Fifteen repetitions of each movement were performed in a randomized order. Movements were performed with both the more and less impaired limb for comparison. Motion of the trunk, arm, forearm, wrist and index fingertip were recorded using a three dimensional active marker motion capture system which recorded movement at a rate of 480 frames per second (Impulse by Phasespace). Motion capture markers were placed on anatomical landmarks of the shoulder, elbow, and wrist, in a manner that allowed for proper post experimentation calculation of joint angles (Robertson et al., 2004).

Muscle activity (EMG) was recorded by a MA-300 EMG system (Motion Lab Systems) using surface electrodes placed over the following twelve muscles: lateral and medial heads of the Triceps Brachii (shoulder extension/ scapular depression), long and short heads of the Biceps Brachii (shoulder flexion/ scapular elevation, elbow flexion, supination), Anterior and Posterior Deltoid (shoulder flexion, extension and abduction), Pectoralis major (shoulder flexion, extension, adduction and medial rotation), Teres Major (shoulder internal rotation), Brachioradialis (elbow flexion), Flexor Carpi Radialis (elbow and wrist flexion), Extensor Carpi Ulnaris (wrist extension), and the Flexor Carpi Ulnaris (wrist flexion)(Winters, 1990). Motion capture and EMG data were recorded simultaneously using a customized circuit and triggering system (Talkington et al., 2015).

\section{Data Analysis}

Recorded data was imported into Matlab and analyzed using custom scripts. Movement onset and offset was identified using a semi-automated approach. The criteria used to determine movement start and stop was an increase or decrease in velocity of greater than five percent of the maximum velocity for that movement. Any errors in the criteria due to inadvertent movement by patients were corrected by a researcher. Joint angles and angular velocity were calculated from the motion capture data for five degrees of freedom including shoulder flexion/extension, shoulder abduction/adduction, shoulder pronation/supination, elbow flexion/extension, and wrist flexion/extension. This kinematic data was then used to calculate limb dynamics using a Simulink model (MathWorks) run in an inverse dynamic mode (for additional description see "Limb Dynamics", chapter 4).

Muscle activity was high pass filtered $(40 \mathrm{~Hz})$, bandpass filtered to remove electrical background noise (59 to $61 \mathrm{~Hz}$ ), rectified, and low pass filtered $(20 \mathrm{~Hz}$ ). Average EMG was then obtained by calculating the mean activity for each muscle across the fifteen repetitions of each movement. Muscle activity was normalized per muscle to the maximum average contraction observed across movement types.

Correlation Analysis 
To examine changes in the muscle activity of chronically impaired stroke patients, we compared muscle activity recorded from the more impaired limb of stroke patients to the average muscle activity recorded from age match controlled subjects. For this comparison, average muscle activity was obtained from the age match control subjects for each muscle for both the right and left limbs. This data was then compared to muscle activity from both the more and less impaired limb of each stroke patient using a regression analysis (Fig. 2). Resulting Pearson's correlation coefficient values ( $\mathrm{R}$-values) from the correlation between the right and left limbs of the control subjects were compared to R-values obtained from the correlation between the more impaired limb of each stroke patient and the corresponding limb of the age match control patients. This analysis was repeated for each movement type and each stroke patient.

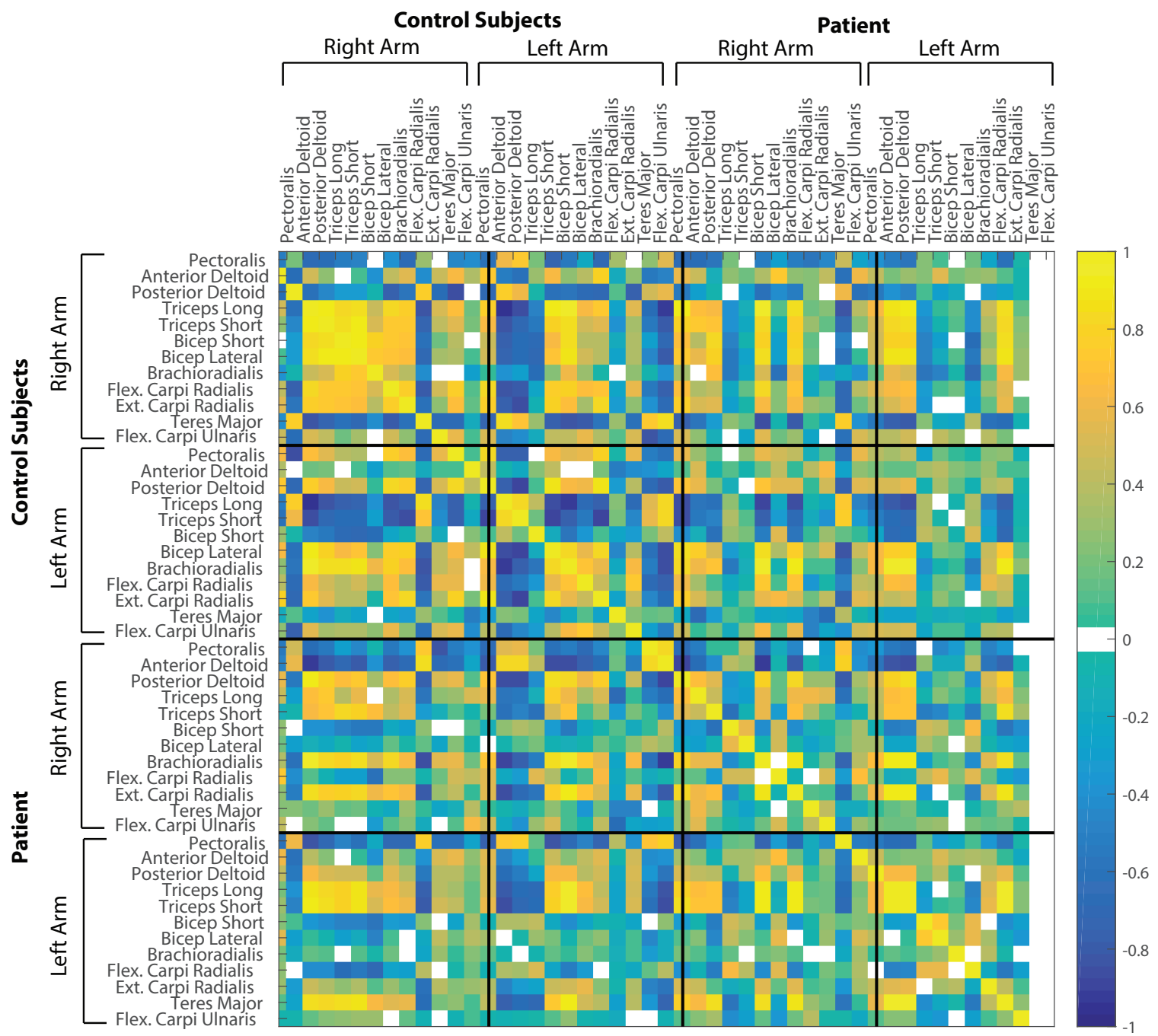

Figure 2: Correlation coefficient plot

Pearson's correlation coefficient was used to compare muscle activity data from the right and left limb of one example subject to average muscle activity from the right and left limb of the age match control subjects for one movement. Darker colors indicate negative correlations, where as Eyellow colors indicate a greater positive correlation. 


\section{Results}

Kinematic and Dynamic signals

Small standard deviations of kinematic signal types were observed across the age match control subjects, suggesting strong similarities in movement (Fig. $3 \mathrm{~A}$ third column, B third column). Movements made by the less impaired limb of stroke patients also showed similar kinematics compared to averages from the age match control group (Fig. 3 A first column, B first column). However, movements made by the more impaired limb of the stroke patients showed significant differences in joint angles and angular velocity when compared to the age match control subjects and the less impaired limb (Fig. 3 A second column, B second column).

Muscle activity across the age match control subjects was also similar, shown by small standard error values (Fig. $3 \mathrm{C}$ third column). Patterns of muscle activation were also similar between the age match control subjects and the less impaired limb of the stroke patients (Fig. $3 \mathrm{C}$ first column). Similar to the kinematic signals, EMG from the more impaired limb of the stroke patients showed significant differences when compared to the less impaired limb and the age matched control subjects (Fig. $3 \mathrm{C}$ second column). As a result of the difference in muscle activity, differences in muscle torques from the more impaired limb of the stroke patients (Fig. $3 \mathrm{D}$ second column) were also observed compared to the age matched controls and the less impaired limb of the stroke patients who had muscle torques similar to each other (Fig. 3 D first and third columns). 
A

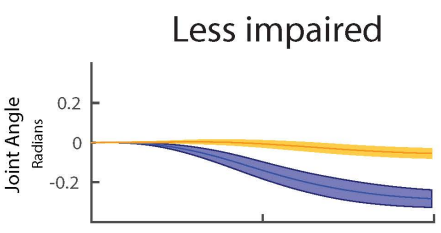

B

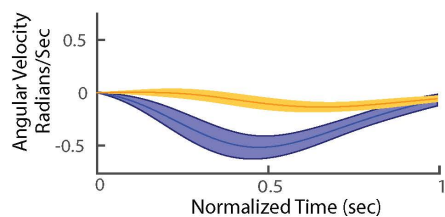

C

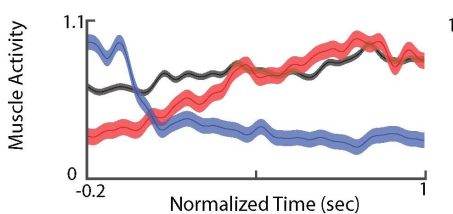

D

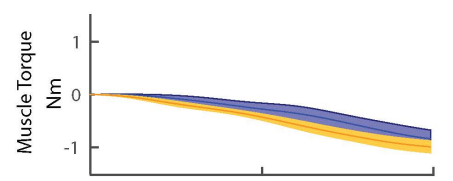

$\mathbf{E}$
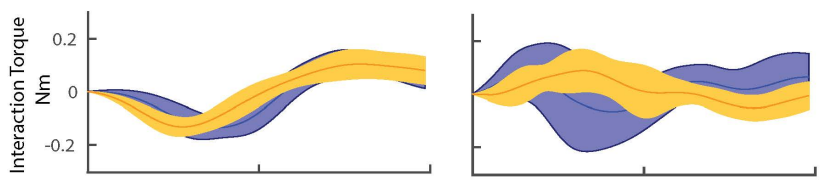

$\mathbf{F}$

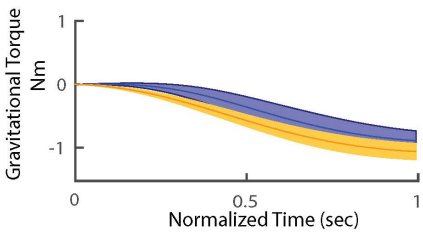

More impaired
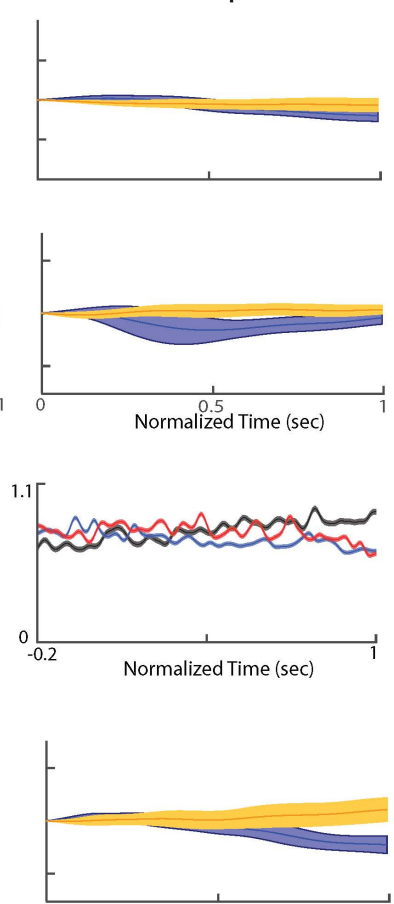

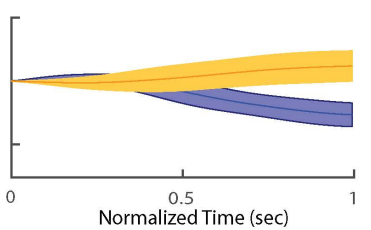

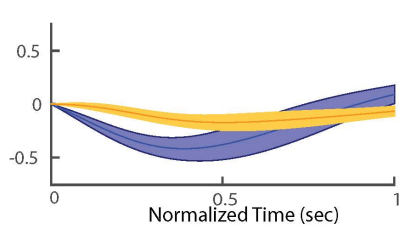
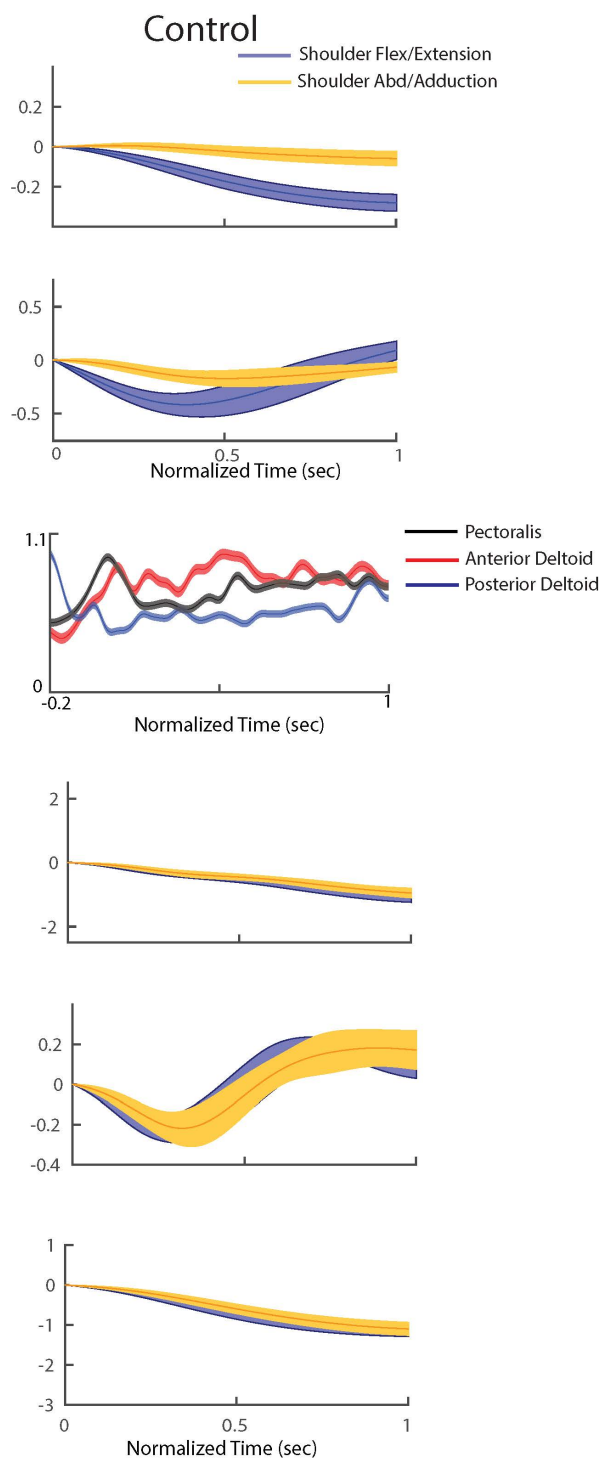

Figure 3: Example kinematic, dynamic, and muscle activity recordings from stroke patients and controls

Data from the more and less impaired limb of one example patient and average data from the age match control subjects for a single movement. (A) Joint angles, and (B) angular velocity, for shoulder abduction/ adduction and flexion/extension. (C) muscle activity recorded from the pectoralis, anterior deltoid, and posterior deltoid. (D) calculated muscle torques, (E) interaction torques, and (F) gravitational torques from custom dynamic model (see methods for more details).

\section{Regression analysis}

Regression analysis comparing the right and left limb of the age match controls across movement types showed high numbers of small correlation values, with decreasing occurrence as correlation values increased (Fig. 4 gray bars). When compared to the more impaired limb of stroke patients, two patients showed less agreement between their more impaired limb and the matching limb from the age 
match control group (Fig. 4, patients 6,7 ). This resulted in a shift of $\mathrm{R}^{2}$ values that clustered closer to 0 , compared to the spread of $\mathrm{R}^{2}$ values seen in the other subjects.
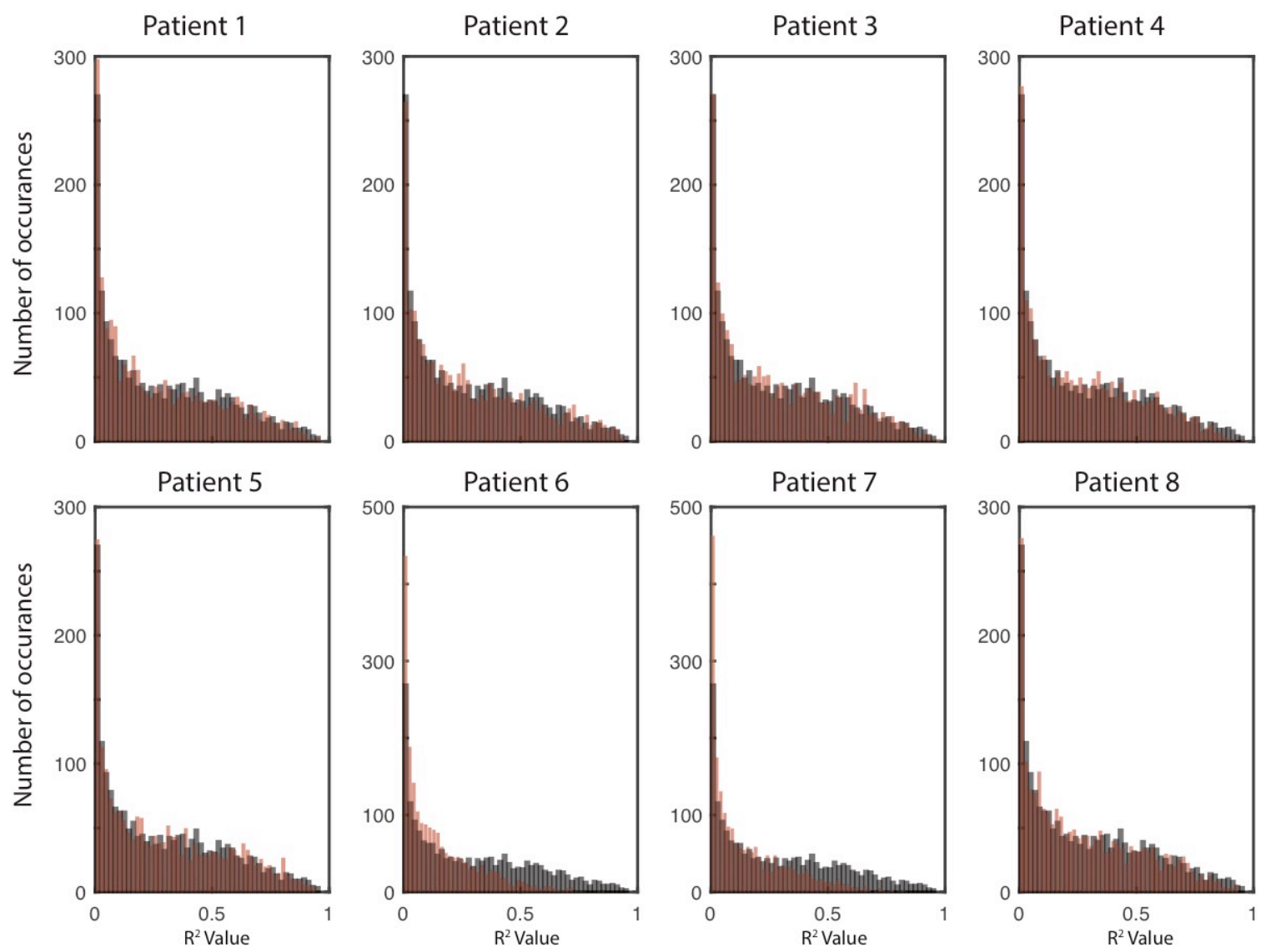

Figure 4: Comparison of $\mathrm{R}^{2}$ values between patients and age match control subjects. Results from the comparison of R-values across all movements for each muscle between patients and age match control averages. Red bars indicate the correlation between the more impaired limb of each stroke patient and the corresponding limb from the age match control subjects. Gray bars indicate the correlation values calculated from the comparison between the right and left limb of the age match controls.

Higher levels of agreement in muscle activity between the limbs of the control subjects were seen for several movement types when compared to the more impaired limb of the patients (Fig. $5 \mathrm{~A}$ ). For six out of the eight patients (patients 1 , $2,4,6,7,8)$ movement type one, had a higher average $\mathrm{R}^{2}$ value for the comparison between the right and left limb of control subjects than the average $\mathrm{R}^{2}$ value for the comparison between the more impaired limb of a stroke patient and the corresponding limb of the control subjects. Movement type three showed average lower $\mathrm{R}^{2}$ values for all eight patients when compared to the correlation between the right and left limbs of the control subjects. For five out of the eight subjects (patients 1, 2, 4, 6, 7), average $\mathrm{R}^{2}$ values from the patient to control comparison were lower for movement four, compared to the inter limb comparison of control subjects. Movement six also had lower average $\mathrm{R}^{2}$ values from the patient to control 
comparison (patients 1, 2, 3, 5, 6, 7); as did movement thirteen (patients 2, 4, 6, 7, 8).
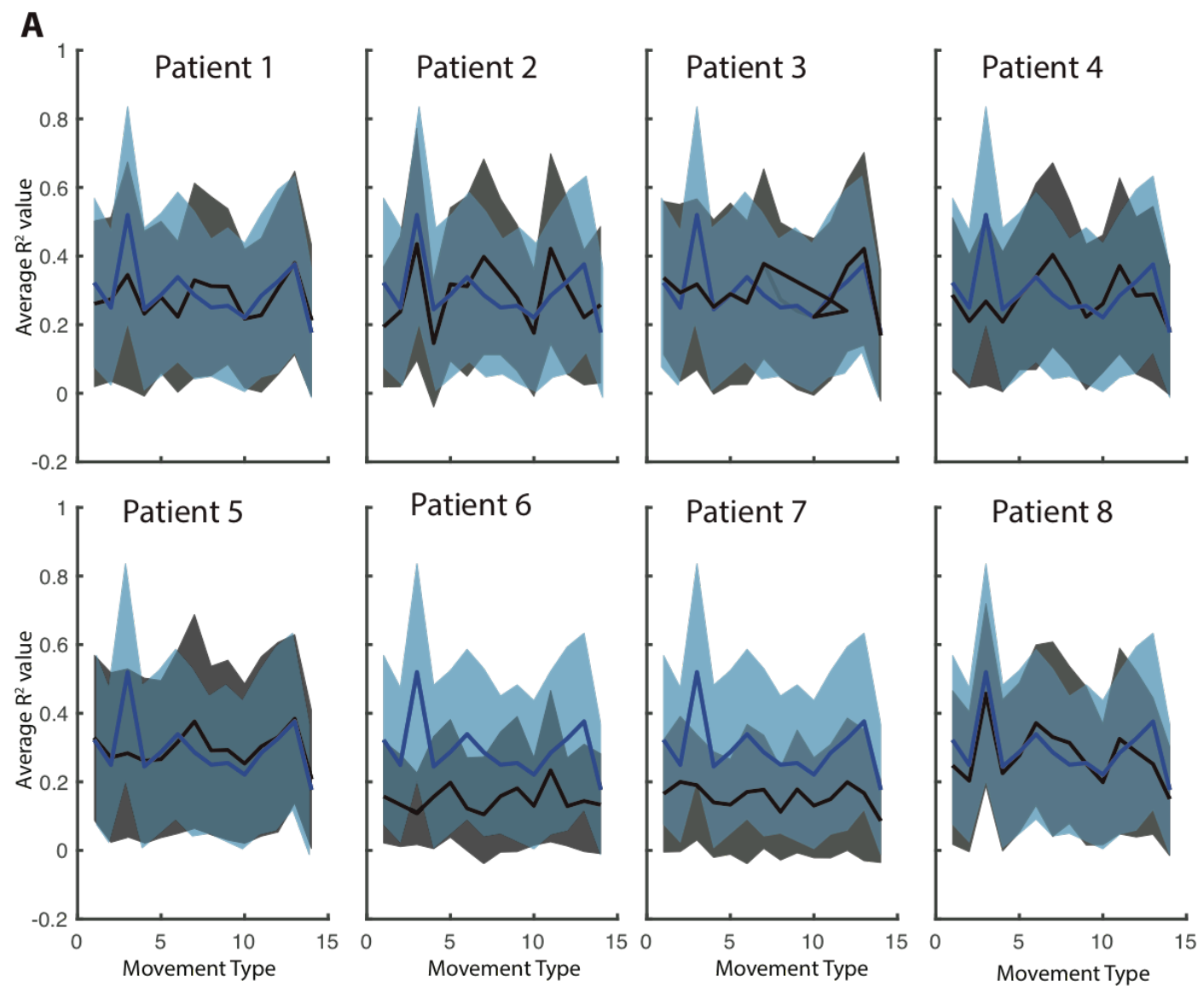

B

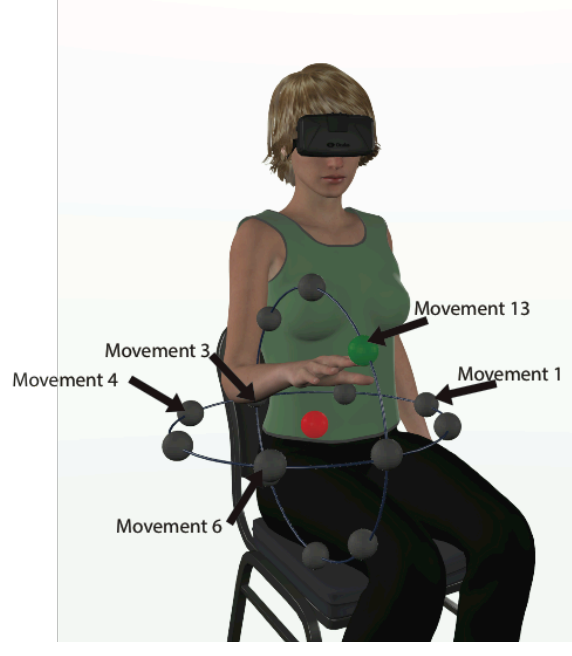

Figure 5: Average $\mathbf{R}^{2}$ values across movement types

(A) Average $\mathrm{R}^{2}$ values for each movement type were calculated from the Pearson's correlation coefficients. Average values (solid lines) for the comparison between the more affected limb of each stroke patient and corresponding limb of the age match controls are displayed in blue with standard deviation (shaded area). Average squared correlation values (solid lines) for the comparison between the right and left limb in the age match control groups are indicated in gray with the shaded area indicating standard deviations. (B) Arrows indicate the movement directions that showed greater differences in muscle activity between the more impaired limb of stroke patients and the corresponding limb of control subjects. 


\section{Discussion}

Here we aimed to examine the relationship between muscle activity and resulting motion in stroke patients who suffer from chronic motor impairment. To closely explore this relationship we had eight stroke survivors perform center out reaching tasks while their motion and muscle activity was recorded. The functional ability of both limbs of the stroke patients was examined and compared to the right and left limb of age match control subjects. This allowed us to examine potential changes or compensatory strategies to the neural control of movement in both the more and less affected limb of the patients.

As in our previous work, we observed strong similarities in joint angles and angular velocity amongst our control subject group. This consistency in the control subjects was also present in muscle activity and dynamic signals. Movement recorded from the less impaired limb of stroke patients also resembled that of the control subjects. This suggests that despite changes to the control of movement for the more impaired limb, normal, or nearly normal, motor control may be preserved for the less impaired limb.

Stark differences were however observed in the more affected limb for several stroke patients. In two out of the eight patients (patients 6 and 7) there was a noticeable decrease in the correlation between muscles of the more affected limb and average muscle activity from the corresponding limb of the control subjects. Interestingly, only one of these patients, patient 6 , showed a significantly slower average movement time on their impaired limb (4.4 seconds, compared to 1.7 seconds on the less impaired limb), where as patient 7 had movement times that were roughly equivalent for both limbs (1.8 and 1.7 seconds) (Table 1 ). This finding suggests that speed of movement, which is often used as a measure of motor impairment in post stroke assessments, may not be a good predictor for the quality of underlying muscle activity. Additionally, patient 8 showed significantly different speeds of movement ( 9.8 seconds for the more impaired limb and 3.8 seconds for the less impaired limb) but had muscle activity that was similar to that of the age matched control subjects.

We also observed several movement types that had lower average $\mathrm{R}^{2}$ values across the patient to subject comparison. Four of these movements were performed in the horizontal plane and one in the vertical plane (see figure $5 \mathrm{~B}$ ). Movements three and four require both shoulder extension and elbow flexion. Movements in this direction create assistive interaction torques at the elbow, which cause passive flexion of the elbow. In this regard, there should be less required muscle activity at the elbow to achieve the desired movement. Movements six and thirteen also create passive interaction torques at the elbow with the extension of the shoulder assisting in the flexion of the elbow.

One could speculate that movements with assistive interaction torques should be easier to complete by individuals with motor impairment due to the passive assistance and thus less required muscle activation. However, it appears that these movements showed the greatest difference in muscle activity between the age match controls and stroke patients.

Previous research into the physiological changes that lead to chronic motor impairment have suggested that ipsilateral motor projections to the paretic side 
may increase during the recovery period of a stroke patient (Turton et al., 1996). These anatomical changes, which arise from plasticity in the central nervous system, have been show to increase abnormal inter joint movement and may be detrimental to movement abilities (Werhahn et al., 2003; Sukal, 2007). This has also been demonstrated as an increase in co-contractions between muscles, causing an increased stiffness to stabilize the limb (Schwerin et al., 2008;). It is possible that those movements, which showed the greatest difference in muscle activity between the age match controls and the stroke patients, may be highlighting the underlying physiological changes that have arisen in the time since the stroke. It is also of interest to note that two out of the eight patients we examined were less than oneyear post stroke (patient 3 who was 6 months post stroke and patient 5 who was 3 months post stroke). Those two patients only showed lower average correlation values for the patient to control comparison for one movement type (movement type 6). It is possible that these two patients are still within an early enough time window since their stroke that the aberrant inter-joint coupling has yet to establish.

Although early, the initial results from this experiment suggest that it may be possible to identify specific movements that are capable of highlighting the altered patterns of muscle activity present in stroke patients. If true, this information would be valuable in helping develop better rehabilitation strategies that focus on the underlying causes of long term motor impairment.

\section{Future directions}

In order to further investigate the changes in motor control that arise after a stroke, several other aspects could be examined within the context of this study. First, it would be of interest to develop a torque metric that could be used to classify movements based on the overall torque load placed on the limb during a movement. This would allow for the comparison between differences in muscle activity to varying levels of overall limb torque. Results from this would provide insight as to how well joint torques are compensated for in stroke patients or if there is a correlation between severity in movement impairment and ability to compensate for different levels of torque.

Another aspect that could provide more information regarding the origin of movement impairment in stroke patients is that of muscle coupling. Particularly, it would be interesting to look at specific muscle couplings that arise during different movements and determine to what extent this coupling is altered in stroke patients. As mentioned before, stroke patients often exhibit higher levels of inter-joint coupling and increased co-contractions. It would be notable to examine this effect in relation to different movement types. If specific movements were identified that showed higher levels of improper muscle coupling then that information would be relevant to developing rehabilitation strategies focused on breaking those deviant co-activations.

The information gained from studies, such as this one, is critical to the progression of scientifically based rehabilitation practices. With added knowledge regarding the changes that arise in muscle activation and the dynamic control of movement in stroke patients, rehabilitation experts can devise better strategies that 
focus on improving the muscle control required to produce correctly executed movements. It is important that researchers and clinicians continue to work towards improving our state of knowledge so that we can provide the best possible long-term outcome for stroke survivors.

\section{Tables}

\begin{tabular}{|l|l|l|l|l|l|}
\hline $\begin{array}{l}\text { Subject } \\
\text { ID }\end{array}$ & Gender & $\begin{array}{l}\text { Infarct } \\
\text { Hemisphere }\end{array}$ & Infarct description and location & $\begin{array}{l}\text { Years post } \\
\text { Stroke }\end{array}$ & $\begin{array}{l}\text { Average movement } \\
\text { time in seconds } \\
\text { (R arm/L arm) }\end{array}$ \\
\hline 1 & M & Right & $\begin{array}{l}\text { Right lateral medullary infarction } \\
\text { with occluded right vertebral artery }\end{array}$ & 8 & $2.4 / 4.1$ \\
\hline 2 & M & Left & $\begin{array}{l}\text { Left caudate lenticular nuclei and } \\
\text { external horn of the left ventricle }\end{array}$ & 5 & $1.9 / 1.5$ \\
\hline 3 & M & Right & $\begin{array}{l}\text { Right dorsal pontine-medullary } \\
\text { lacunar infarction }\end{array}$ & 6 months & $2.4 / 2.4$ \\
\hline 4 & F & Left & $\begin{array}{l}\text { Left middle cerebral artery } \\
\text { Right }\end{array}$ & $\begin{array}{l}\text { Lacunar infarct involving } \\
\text { posterior right putamen and } \\
\text { border of right internal capsule }\end{array}$ & 3 \\
\hline 6 & M & Right & Right middle cerebral artery & 11 & $1.9 / 1.8$ \\
\hline 7 & M & Right & Right middle cerebral artery & 6 & $1.5 / 1.5$ \\
\hline 8 & M & Right & $\begin{array}{l}\text { Right middle cerebral artery, } \\
\text { extending posteriorly. }\end{array}$ & 7 & $3.8 / 2.5$ \\
\hline
\end{tabular}

Table 1: Stroke patient demographics and information

Eight chronically motor impaired stroke patients were recruited from Ruby Memorial Hospital. Stroke location was identified from imaging reports (MRI or CT) and confirmed by the treating physician. Average movement time was calculated from kinematics recorded during the fifteen repetitions of the fourteen movements. 


\section{Chapter 6}

Summary and recommendations for future research 
Despite its necessity to everyday life, there remains much unknown regarding the control of movement. This gap in knowledge is continually studied in the ever-expanding field of motor control research, leading to the development of new methods and theories that aim to explain the control of our vastly complex musculoskeletal system. The application of this growing body of knowledge ranges from the improved understanding of neural networks to the development of new therapies for motor impaired individuals. And yet, despite our vast improvement in what is known about the motor control of humans, there still remain many unanswered questions and areas of contention.

A reasonable answer to why so many different theories of motor control are still hotly debated lies within the complexity of the system being studied. Human bodies are intricately developed systems, with dependencies between the bones, which form our main structure, and the muscles that drive the motion of those bones. Because of these mutually contingent parts, it becomes difficult to separate out what aspects of movement are planned verses which aspects of movement occur as a result from our biomechanical framework. This embedded artifact of human motion creates a difficult system to study. Adding to this difficulty is the wide variety of movements humans can perform. Just a simple reaching movement can involve greater than five degrees of freedom, tens of muscles, bones, and accompanying joints. Add to this, differences in speed, accuracy, and distances and the number of possible tasks that can be tested seems endless.

The methods used to study the human motor control system also create a confounding influence on the answers that arise from experiments. Experiments that focus on measurements of muscle activity provide answers regarding the coordination of muscles necessary to drive the joints and bones in the appropriate manner. Yet, these studies can overlook the interplay between muscles, dynamics and the resulting kinematics. Conversely, studies that examine kinematics are shortsighted in observing only the resulting movement and not the underlying forces that produced that movement. This limitation lead us to develop an experiment where both muscle activity and the accompanying kinematics and dynamics could be studied. The goal of this work, described in chapter four, was to determine if motor primitives derived from muscle activity matched motor primitives from movement kinematics and dynamics. Our primary metric of comparing muscle motor primitives to kinematic and dynamic based primitives was the comparison of how the weights associated with each primitive type were distributed across movement types. Prior work in this field has shown that primitive weights are spatially tuned in specific ways. We therefor hypothesized that if kinematic and dynamic information affects the structure of muscle based primitives then the tuning of these different primitive types should be equivalent. Interestingly, we found that there was in fact no significant overlap between kinematic, dynamic, and muscle based primitives. These findings lead to the rejection of our main hypothesis. This is not to say that there is a devisable relationship between muscle activity, dynamics, and kinematics. It is however possible that this relationship may not be fully captured from the procedures of dimensional data deconstruction often used to classify primitives. For the future of motor primitive research, it would be imperative to find a method of primitive 
classification that accounts for the relationship between kinematics, dynamics, and muscle activity.

Improved methodology for investigating this relationship would also be greatly beneficial to the application of studying chronic motor impairment in stroke patients. A great deal of effort has focused on creating a more complete and easy to apply metric for quantifying post stroke motor impairment. However, significant limitations have derailed these efforts. These limitations can be broken down into two categories: method of observation and data collected. First, the method of observing motor impairment in stroke patients has been previously limited to the ability of a trained physical therapist to visually score movement. This provides a qualitative measure of motor impairment but does not provide a quantitative score. Additionally, the use of these tests and the quality of a report generated from these tests is reliant on the expertise of the administering clinician. To combat this issue, research, research over the last decade has aimed to quantify movement impairment by using state of the art technology such as motion capture equipment. Although solving the issue of providing a quantitative measure, this equipment is expensive and difficult to use by an untrained individual, making it irrelevant in the clinical setting. As an alternative to this approach, we have tested the use of low cost motion capture equipment that provides a "plug and play" easy to use set up. This set up works well for movements in the frontal plane but was found to be not as well suited for movements that crossed multiple planes or incorporated fine motor movements, such as picking up paper clips. However, this approach of applying low cost motion capture to the quantification of motor impairment should not be abandoned by these shortcomings. The speed with which this technology is advancing is very promising and it is likely that this technology will be clinically adaptable within the next ten years.

This technology, however, will need to address the other limitation currently imposed on clinical measures of motor impairment, which is the type of data currently used to describe post stroke movement. Kinematic data, such as joint angles, velocity, and acceleration are important metrics but fall short in capturing all aspects of movement impairment. For clinicians to have a full description of motor impairment, it is important for muscle activity to be accounted for as well. This however is a difficult request to accommodate. Recording muscle activity requires the use of EMG, and although this technology is becoming cheaper and easier to use, its application and analysis still requires a trained individual. The best solution to this would be to provide clinicians with a tool that could predict muscle activity from kinematic data. Programs such as OpenSim, created by researchers at Stanford, are the early stages of what could be a clinically relevant tool. Research being conducted at Microsoft has also produced an early stage model of predicting muscle activity from movement, but there remains considerable work to create a model that is adaptable to patients with movement impairment.

The work completed during the tenure of this dissertation aimed to address some of the critical gaps in our knowledge regarding motor control and its application to studying post stroke motor deficits. Additionally, this work intended to push the boundaries of clinically relevant tools that would provide clinicians a more comprehensive mechanism for quantifying post stroke movement 
impairment. For the future care of stroke patients it is crucial that these topics continue to be investigated. Equally important is that the dialogue between researchers and clinicians remain open and effective. This will encourage the transfer of research findings into clinically relevant tools, which is the only way to provide improved care to this important and growing patient population. 


\section{References}

Abend W, Bizzi E and Morasso. Human arm trajectory formation. Brain; 1982: 331348

Adams H, Zoppo G, Alberts M, Bhatt D, Brass L, Furlan A, Grubb R, Higashida R, Jauch E, Kidwell C, Lyden P, Morgenstern L, Qureshi A, Rosenwasser R, Scott P, Wijdicks E. American Heart Association, Guidelines for the early management of adults with ischemic stroke. 2007. DOI: 10.1161/CIRCULATIONAHA.107.181486

An K and Chao E. Kinematic analysis of human movement. Anals of Biomedical Engineering. 1985; 12: 585-597

American Heart Association. Heart Disease and Stroke Statistics. 2016 Fact Sheet. Updated, 2017; available at https://www.heart.org/HEARTORG/General/Heartand-Stroke-Association-Statistics_UCM_319064_SubHomePage.jsp

Atkenson C and Hollerbach F. Kinematic features of unrestrained vertical arm movements. Journal of neuroscience. 1985; 9: 2318-2330

Beer RF, Dewald JPA, Rymer WZ. Deficits in the coordination of multi- joint arm movements in patients with hemiparesis: evidence for dis- turbed control of limb dynamics. Exp Brain Res 2000; 131: 305-19.

Bernstein, N. (1967) The Co-ordination and Regulation of Movements. Oxford, New York. Pergamon Press.

Bizzi E, Edelman G, Changeux J. (2001) The Brain. New Brunswick, New Jersey. Transaction Publishers.

Bizzi E, Mussa-Ivaldi F and Giszter S. Computations underlying the execution of movement: a biological perspective. Science. 1991; 253: 287-291

Bizzi E and Cheung V. The neural origin of muscle synergies. Frontiers in computational neuroscience. 2013; 7: 1-6

Bockemuhl T, Troje N, Durr V. Inter-joint coupling and joint angle synergies of human catching movements. Human Movement Science. 2010; 29: 73-93

Chang YJ, Chen SF, Huang JD. A Kinect-based system for physical rehabilitation: A pilot study for young adults with motor disabilities. Research in Developmental Disabilities. 2011; 32, 2566-2570.

Cheung V, Piron L, Agostini M, Silvoni S, Turolla A, Bizzi E. Stability of muscle synergies for

voluntary actions after cortical stroke in humans. PNAS. 2009; 106: 19563-568 
Cheung V, Turolla A, Agostini M, Silvoni S, Bennis C, Kasi P, Paganoni S, Bonato P, Bizzi E. Muscle synergy patterns as physiological markers of motor cortical damage. PNAS. 2012; 109: 14652-656

Clark DJ, Ting LH, Zajac FE, Neptune RR, Kautz SA. Merging of healthy motor modules predicts reduced locomotor performance and muscle coordination complexity post-stroke. J. Neurophysiol. 2010: 103; 844-857.

Cruz T, Dhaher Y. Evidence of abnormal lower limb torque synergies after stroke: an isometric study. Stroke. 2008;39:139-147

d'Avella A and Lacquaniti F. Control of reaching movements by muscle synergy combinations. Frontiers in Computational Neuroscience. 2013; doi:

$10.3389 /$ fncom.2013.00042

d'Avella A, Fernandez L, Portone A, Lacquaniti F. Modulation of phasic and tonic muscle synergies with reaching direction and speed. Journal of Neurophysiology. 2008; 100: 1433-1454

Dewald JP, Pope PS, Given JD, Buchanan TS, Rymer WZ. Abnormal muscle coactivation patterns during isometric torque generation at the elbow and shoulder in hemiparetic subjects. Brain 1995; 118: 495-510.

Dipietro L, Krebs HI, Fasoli SE, Volpe BT, Stein J, Bever C, Hogan N. Changing motor synergies in chronic stroke. Journal of neurophysiology. 2007; 98: 757-768.

Dobkin B. Rehabilitation after Stroke. New England Journal of Medicine. 2005; 352:1677-1684

Duncan PW, Goldstein LB, Matchar D, Divine GW, Feussner J. Measurement of motor recovery after stroke: Outcome assessment and sample size requirements. Stroke. 1992; 23(8):1084-1089.

Feys HM, De Weerdt WJ, Selz BE Cox GA, Spinchiger R, Vereeck LE, Putman KD, Van Hoydonck GA. Effect of a therapeutic intervention for the hemiplegic upper limb in the acute phase after stroke: a single-blind, randomized, controlled multicenter trial. Stroke. 1998; 29(4):785-92.

Forner-Cordero A, Levin O, Li Y, Swinnen SP. Principal component analysis of complex multijoint coordinative movements. Biological Cybernetics; 2005: 93, 6378.

Fugl-Meyer AR, Jaasko L, Leyman I, Olsson S, Steglind S. The post-stroke hemiplegic patient. A method for evaluation of physical performance. Scandinavian Journal of Rehabilitation Medicine. 1975;7:13-31. 
Giszter SF. Motor primitives-new data and future questions. Current Opinion in Neurobiology. 2015; 33: 156-165

Gowland C, deBruin H, Basmajian JV, Plews N, Burcea I. Agonist and antagonist activity during voluntary upper-limb movement in patients with stroke. Phys Ther 1992; 72: 624-33.

Gribble P and Ostry D. Compensation for interaction torques during single and multi-joint limb movement. J Neuro-physiol. 1999; 82: 2310-2326

Grinyagin IV, Biryukova EV, Maier MA. Kinematic and dynamic synergies of human precision-grip movements. Journal of Neurophysiology. 2005; 94: 2284-2294.

Hamill J, Knutzen K. (2009) Biomechanical Basis of Human Movement, third edition. Baltimore, MD. Lippincott, Williams and Wilkins.

Hirashima M, Kudo K, Wateral K, Ohtsuki T. Control of 3D limb dynamics in unconstrained overarm throws of different speeds performed by skilled baseball players. J Neurophysiol. 2007; 97: 680-691

Hollerback JM and Flash T. Dynamic interactions between limb segments during planar arm movement. Biol. Cybern. 1982; 44: 67-77

Kim SY, Allred RP, Adkins DL, Tennant KA, Donlan NA, Kleim JA, Jones TA. Experience with the "Good" Limb induces aberrant synaptic plasticity in the perilesion cortex after stroke. The Journal of Neuroscience. 2015;

DOI: 10.\%u200B1523/\%u200BJNEUROSCI.\%u200B0829-15.\%u200B2015

Knorr B, Hughes R, Sherrill D, Stein J, Akay M, Bonato P. Quantitative measures of functional upper limb movement in persons after stroke. IEEE. 2005;252-255

Krakauer J. Arm function after stroke: from physiology to recover. Seminars in neurology. 2005; 25:384-95

Krakauer J, Carmichael ST, Corbett D, Wittenberg G. Getting neurorehabilitation right: what can be learned from animal models? Journal of Neurorehabilitation and Neural Repair. 2012; 26(8): 923-931

Lange B. et al. Development and evaluation of low cost game-based balance rehabilitation tool using the Microsoft Kinect sensor. Annual International Conference of the IEEE Engineering in Medicine and Biology Society IEEE Engineering in Medicine and Biology Society Conference 2011, 1831-1834. Langhorne P, Coupar F, Pollock A. Motor recovery after stroke: a systematic review. Lancet Neurology. 2009; 8: 741-754. 
Lemay M and Grill W. Modularity of motor output evoked by intraspinal microstimulation in cats. Journal of Neurophysiology. 2004; 91: 502-514

Levin MF. Interjoint coordination during pointing movements is disrupted in spastic hemiparesis. Brain. 1996; 119: 281-93.

Lough S, Wing AM, Fraser C, Jenner JR. Measurement of recovery of function in the hemiparetic upper limb following stroke: a preliminary report.

Hum Mov Sci 1984; 3: 247-56.

Mathie M, Coster A, Lovell N, Celler B. Accelerometry: providing an integrated, practical method for long-term, ambulatory monitoring of human movement. Physiol Meas. 2004; 25: 1-20

Morasso P. Spatial control of arm movements. Experimental Brain Research. 1982; 42: $223-227$

Nakayama H, Jørgensen HS, Raaschou HO, Olsen TS. Recovery of upper extremity function in stroke patients: the Copenhagen stroke study. Arch. Phys. Med. Rehabil. $1994 ; 75,394-398$

Neckel N, Pelliccio M, Nichols D, Hidler J. Quantification of functional weakness and abnormal synergy patterns in the lower limb of individuals with chronic stroke. Journal of NeuroEngineering and Rehabilitation. 2006; 3: 3-17

Patel S, Lorincz K, Hughes R, Huggins N, Growdon J, Standaert D, Akay M, Dy J, Welsh $\mathrm{M}$, Bonato P. Monitoring motor fluctuations in patients with Parkinson's disease using wearable sensors. IEEE Transfer Inf Technology Biomedical. 2009; 13: 864873

Perreault EJ, Chen K, Trumbower RD, Lewis G. Interactions with compliant loads alter stretch reflex gains but not intermuscular coordination. J Neurophysiol 2008; 99: 2101-2113.

Raibert M. Motor control and learning by the state space model. Artificial intelligence laboratory, Massachusetts Institute of Technology, Robotics. 1977; 761

Robertson G, Caldwell G, Hamill J, Kamen G, Whittlesey S. Research Methods in Biomechanics, second edition. Champaign, IL: Human Kinetics; 2014.

Roh J, Rymer WZ, Perreault EJ, Yoo SB, Beer RF. Alterations in upper limb muscle synergy structure in chronic stroke surivors. Journal of neurophysiology. 2013; 109: 768-781. 
Roh J, Rymer WZ, Beer RF. Evidence for altered upper extremity muscle synergies in chronic stroke survivors with mild and moderate impairment. Frontiers in Human Neuroscience. 2015; 10.3389/fnhum.2015.00006

Safavynia SA, Torres-Oviedo G, Ting LH. Muscle Synergies: Implications for Clinical Evaluation and Rehabilitation of Movement. Topics in spinal cord injury and rehabilitation. 2011; 17: 16-24

Safavynia S and Ting L. Sensorimotor feedback based on task-relevant error robustly predicts temporal recruitment and multidirectional tuning of muscle synergies. Journal of Neurophysiology. 2012; 109(1): 31-45

Sainburg R, Ghilardi M, Poizner G and Ghez C. Control of limb dynamics in normal subjects and patients without proprioception. J Neurophysiol. 1995; 73: 820-835

Schneider K, Zernicke R, Schmidt R, Hart T. Changes in limb dynamics during the practice of rapid arm movements. J. Biomechanics. 1989; 22: 805-817

Sergio L and Kalaska J. Systematic changes in directional tuning of motor cortex cell activity with hand location in the workspace during generation of static isometric forces in constant spatial directions. The American Physiological Society. 1997; $1170-1174$

Soechting J and Lacquaniti F. Invariant characteristics of a pointing movement in response to a chance in target location. Journal of Neurophysiology. 1981; 1: 710720

Steele KM, Tresch MC, Perreault EJ. The number and choice of muscles impact the results of muscle synergy analyses. Frontiers in Computational Neuroscience. 2013; doi: $10.3389 /$ fncom.2013.00105

Stinear C, Acherley S, Byblow W. Rehabilitation is Initiated Early After Stroke, but Most motor Rehabilitation Trials are Not. Stroke. 2013; 44:2039-2045.

Stone E, Skubic M. Passive in-home measurement of stride-to-stride gait variability comparing vision and Kinect sensing. Annual International Conference of the IEEE Engineering in Medicine and Biology Society IEEE Engineering in Medicine and Biology Society Conference 2011, 6491-6494

Subramanian SK, Yamanaka J, Chilingaryan G, Levin M. Validity of movement pattern kinematics as measures of arm motor impairment post stroke. Stroke. 2010. $41 ; 10: 2303-2308$.

Sukal TM, Ellis MD, Dewald JPA. Shoulder abduction induced reductions in reaching work area following hemiparetic stroke: neuroscientific implications. Experimental Brain Research. 2007; 183: 215-223. 
Takeuchi N, Izumi SI. Maladaptic plasticity for motor recovery after stroke: mechanisms and approaches. Neural Plasticity. 2012; 10.1155/2012/359728

Talkington W, Pollard B, Olesh EV, Gritsenko V. Multifunctional setup for studying human motor control using transcranial magnetic stimulation, electromyography, motion capture, and virtual reality. Journal of Visualized Experiments. 2015.

Tropea P, Monaco V, Coscia M, Posteraro F, Micera S. Effects of early and intensive neuro-rehabilitative treatment on muscle synergies in acute-post stroke patients: a pilot study. Journal of Neural Engineering and Rehabilitation. 2013;

DOI: $10.1186 / 1743-0003-10-103$

Thies S, Tresadern L, Kenney LP, Smith J, Howard D, Goulermas JY, Smith C, Rigby J. Movement variability in stroke patients and controls performing two upper limb functional tasks: a new assessment methodology. Journal of Neuroengineering and rehabilitation. 2009; 10.1186/1743-0003-6-2

Ting LH. Dimensional reduction in sensorimotor systems: a framework for understanding muscle coordination of posture. Progress in brain research. 2007; 165: 299-321

Torres-Oviedo G, Ting LH. Muscle synergies characterizing human postural responses. J Neurophysiol 2007; 98:2144-2156

Tresch MC, Bizzi E. Responses to microstimulation in the chronically spinalized rat and their relationship to spinal systems activated by low threshold cutaneous systems. Experimental Brain Research. 1999; 129:401-416.

Tresch M, Saltiel P, d'Avella A, and Bizzi E. Coordination and localization in spinal motor systems. Brain Res. Rev. 2002; 40:66-79.

Tresch M, Cheung V and d'Avella A. Matrix factorization algorithms for the identification of muscle synergies: evaluation on simulated and experimental data sets. Journal of Neurophysiology. 2006; 95: 2199-2212

Trombly CA. Deficits of reaching in subjects with left hemiparesis: a pilot study. Am J Occup Ther 1992; 46: 887-97.

Tura A, Rocchi L, Raggi M, Cutti A, Chiari L. Recommended number of strides for automatic assessment of gait symmetry and regularity in above-knee amputees by means of accelerometry and autocorrelation analysis. Journal of NeuroEngineering and Rehabilitation. 2012; 9: 9-11

Turton A, WroeS, Trepte N, Fraser C, Lemon RN, “Contralateral and ipsilateral EMG responses to transcranial magnetic stimulation during recovery of arm and hand 
function after stroke," Electroencephalography and Clinical Neurophysiology. 1996; 101: 316-328

Wade D. Measurement in neurological rehabilitation. Oxford, England: Oxford University Press; 1992

Wahl AS, Schwab M. Finding an optimal rehabilitation paradigm after stroke: enhancing fiber growth and training of the brain at the right movement. Frontiers in Human Neuroscience. 2014; doi: 10.3389/fnhum.2014.00381

Warlow C, van Gijn J, Dennis M, et al. Stroke: practical management, third edition. Oxford, England: Blackwell Publishing; 2008

Werhahn KJ, Conforto AB, Kadom B, Hallet M, Cohen LG. Contributions of the ipsilateral motor cortex to recovery after chronic stroke. Annals of Neurology. 2003; 54: 464-472.

Winter D. Biomechanics of human movement. Hoboken, New Jersey: John Wiley; 1990

Wolf S, Catlin P, Ellis M, Archer A, Morgan B, Piacentino A. Assessing the Wolf Motor Function Test as Outcome Measure for Research in Patients After Stroke. Stroke. 2001;32, 1635-1639. 